\title{
Algebraic $K$-theory, algebraic cycles and arithmetic geometry
}

\author{
Bruno Kahn \\ Institut de Mathématiques de Jussieu \\ 175-179 rue du Chevaleret \\ 75013 Paris \\ France \\ kahn@math.jussieu.fr
}

\section{Introduction}

Warning: This paper is full of conjectures. If you are allergic to them it may be harmful to your health. Parts of them are proven, though.

In algebraic geometry, one encounters two important kinds of objects: vector bundles and algebraic cycles. The first lead to algebraic $K$-theory while the second lead to motivic cohomology. They are related via the Chern character and Atiyah-Hirzebruch-like spectral sequences.

Vector bundles and algebraic cycles offer very different features. On the one hand, it is often more powerful and easier to work with vector bundles: for example Quillen's localisation theorem in algebraic $K$-theory is considerably easier to prove than the corresponding localisation theorem of Bloch for higher Chow groups. In short, no moving lemmas are needed to handle vector bundles. In appropriate cases they can be classified by moduli spaces, which underlies the proof of finiteness theorems like Tate's theorem [189] and Faltings' proof of the Mordell conjecture, or Quillen's finite generation theorem for $K$-groups of curves over a finite field [66]. They also have a better functoriality than algebraic cycles, and this has been used for example by Takeshi Saito in [163, Proof of Lemma 2.4.2] to establish functoriality properties of the weight spectral sequences for smooth projective varieties over $\mathbf{Q}_{p}$.

On the other hand, it is fundamental to work with motivic cohomology: as $E_{2}$-terms of a spectral sequence converging to $K$-theory, the groups involved contain finer torsion information than algebraic $K$-groups (see Remark 2.2.2), they appear naturally as Hom groups in triangulated categories of motives and they appear naturally, rather than $K$-groups, in the arithmetic conjectures of Lichtenbaum on special values of zeta functions.

In this survey we shall try and clarify for the reader the interaction between these two mathematical objects and give a state of the art of the (many) 
conjectures involving them, and especially the various implications between these conjectures. We shall also explain some unconditional results.

Sections 1 to 4 are included for the reader's convenience and are much more developed in other chapters of this Handbook: the reader is invited to refer to those for more details. These sections are also used for reference purposes. The heart of the chapter is in Sections 6 and 7: in the first we try and explain in much detail the conjectures of Soulé and Lichtenbaum on the order of zeroes and special values of zeta functions of schemes of finite type over $\operatorname{Spec} \mathbf{Z}$, and an approach to prove them, in characteristic $p$ (we don't touch the much more delicate Beilinson conjectures on special values of $L$-functions of $\mathbf{Q}$-varieties and their refinements by Bloch-Kato and Fontaine-Perrin-Riou; these have been excellently exposed in many places of the literature anyway). In the second, we indicate some cases where they can be proven, following [95].

There are two sources for the formulation of (ii) in Theorem 6.7.1. One is Soulé's article [175] and the article of Geisser building on it [56], which led to the equivalence of (ii) with (i). The other source is the formulation of the Beilinson-Lichtenbaum conjecture and its treatment by Suslin-Voevodsky in (the first version of) [186], which led to the equivalence of (ii) with (iii). As the Bloch-Kato conjecture and the Beilinson-Lichtenbaum conjecture both take their roots in number theory and arithmetic geometry, it is a bit of a paradox that they seem irrelevant to the conjectures on special values of zeta functions after all: see the discussion in Subsection 8.1.

I have tried to keep the exposition as light as possible without sacrificing rigour. This means that many proofs are omitted and replaced by references to the literature. Many others are just sketches, although hopefully these should be enough for the interested reader to reconstitute them. Some proofs are complete, though. I hope this will not create any frustration or confusion.

I wish to thank Yves André, Antoine Chambert-Loir, Pierre Colmez, Thomas Geisser, Dan Grayson, Manfred Kolster, Max Karoubi, Fabien Morel, Thong Nguyen Quang Do, Joël Riou, Jean-Pierre Serre, V. Srinivas, Chuck Weibel and the referee for helpful comments on the first versions of this text. This work was partly revised during a stay in TIFR, Mumbai, under CEFIPRA Project 2501-1 Algebraic groups in arithmetic and geometry, and I gratefully acknowledge its support.

\section{Contents}

1 The picture in algebraic topology $\ldots \ldots \ldots \ldots \ldots \ldots \ldots \ldots$

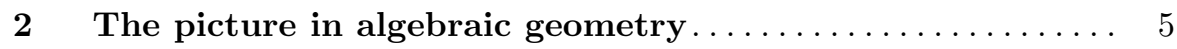

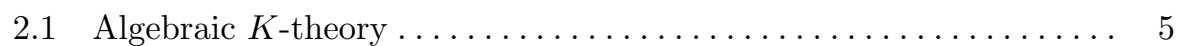

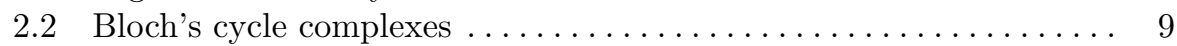

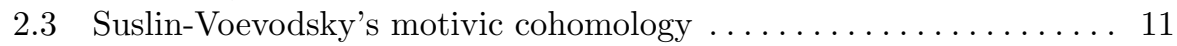

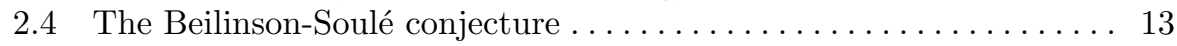


3 Review of motives $\ldots \ldots \ldots \ldots \ldots \ldots \ldots \ldots \ldots \ldots \ldots \ldots$

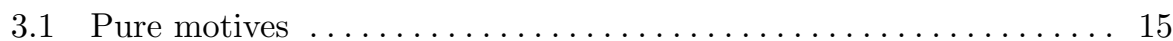

3.2 The conjectural abelian category of mixed motives . . . . . . . . 19

3.3 The nonconjectural triangulated categories of mixed motives . . . . 20

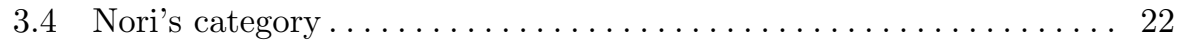

$3.5 \mathbf{A}^{1}$-homotopy and stable homotopy categories ............ 23

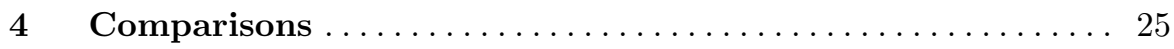

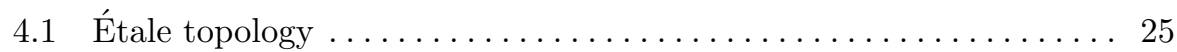

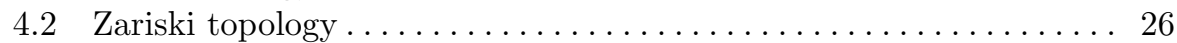

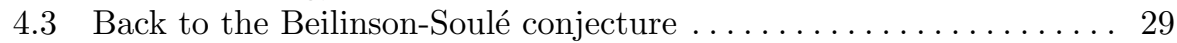

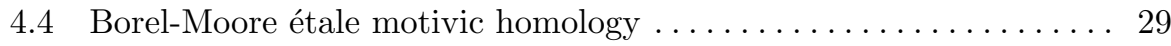

5 Applications: local structure of algebraic $K$-groups and finiteness theorems .................... 32

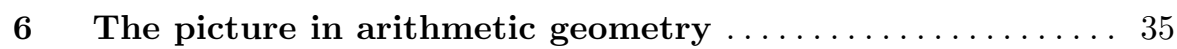

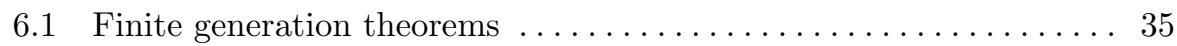

6.2 Ranks, torsion and zeta functions $\ldots \ldots \ldots \ldots \ldots \ldots \ldots \ldots \ldots$

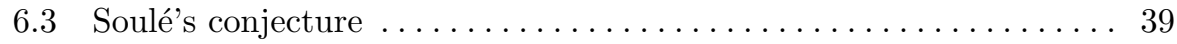

6.4 Lichtenbaum's first conjecture: rings of algebraic integers . . . . . . . 41

6.5 The Tate, Beilinson and Parshin conjectures . . . . . . . . . . . . 44

6.6 Lichtenbaum's second conjecture: varieties over finite fields . . . . . . 45

6.7 Motivic reformulation of the Tate and Beilinson conjectures . . . . 47

6.8 Lichtenbaum's Weil-étale topology; reformulation of his second

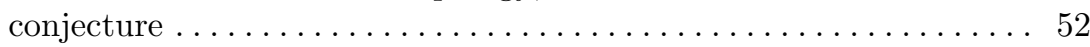

7 Unconditional results: varieties of abelian type over finite

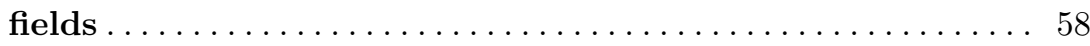

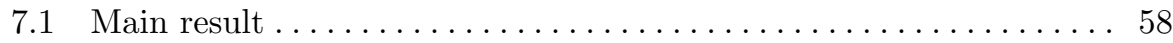

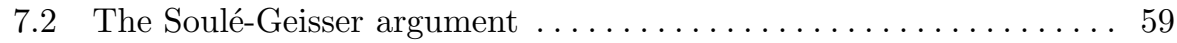

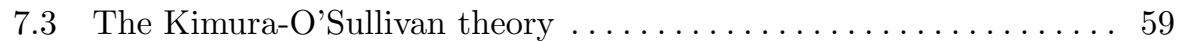

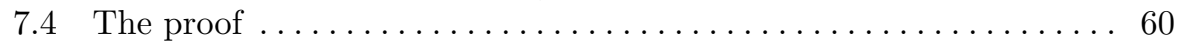

7.5 Justification of Example 7.1 .21$) \ldots \ldots \ldots \ldots \ldots \ldots \ldots \ldots \ldots \ldots \ldots \ldots$

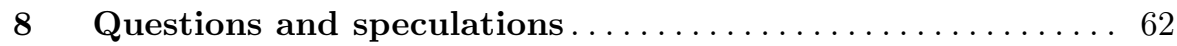

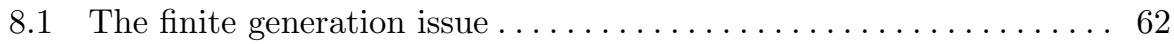

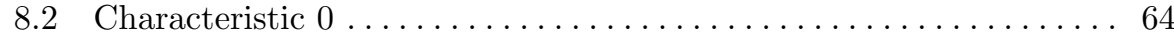

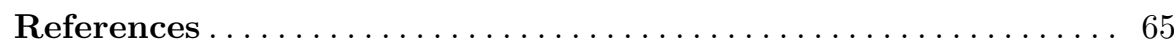

\section{The picture in algebraic topology}

(For complements on this section, see Geisser's chapter, §4, Karoubi's chapter in this Handbook and also [99, ch. V].) 
The picture in algebraic topology is quite simple: singular cohomology and complex $K$-theory are related both via the Atiyah-Hirzebruch spectral sequence and via the Chern character. The latter lets the Atiyah-Hirzebruch spectral sequence degenerate rationally, something the Adams operations also perform.

More precisely, let $X$ be a reasonable topological space: say, $X$ has the homotopy type of a $C W$-complex. The singular cohomology of $X, H^{*}(X, \mathbf{Z})$, is the cohomology of the cochain complex $\operatorname{Hom}\left(C_{*}(X), \mathbf{Z}\right)$, where

$$
C_{i}(X)=\mathbf{Z}\left[\mathcal{C}^{0}\left(\Delta^{i}, X\right)\right]
$$

where $\mathcal{C}^{0}$ denotes continuous functions and $\Delta^{i}$ is the standard $i$-simplex. The differential $C_{i}(X) \rightarrow C_{i-1}(X)$ is defined as the alternating sum of the restrictions of a given map to $(i-1)$-dimensional faces. The functors $H^{i}(-, \mathbf{Z})$ are representable in the sense that

$$
H^{i}(X, \mathbf{Z})=[X, K(\mathbf{Z}, i)]
$$

where $K(\mathbf{Z}, i)$ is the $i$-th Eilenberg-Mac Lane space of $\mathbf{Z}$.

On the other hand, complex $K$-theory of $X$ may be defined as

$$
K^{i}(X)= \begin{cases}{[X, \mathbf{Z} \times B U]} & \text { if } i \text { is even } \\ {[X, U]} & \text { if } i \text { is odd }\end{cases}
$$

where $U$ is the infinite unitary group. Bott periodicity gives canonical isomorphisms $K^{i}(X) \simeq K^{i+2}(X)$. If $X$ is compact, $K^{0}(X)$ (stably) classifies complex vector bundles over $X$.

The Atiyah-Hirzebruch spectral sequence has the following form:

$$
E_{2}^{p, q}=\left\{\begin{array}{ll}
H^{p}(X, \mathbf{Z}) & \text { if } q \text { is even } \\
0 & \text { if } q \text { is odd }
\end{array} \Rightarrow K^{p+q}(X)\right.
$$

while the Chern character has the form

$$
\begin{aligned}
& c h: K^{0}(X) \otimes \mathbf{Q} \rightarrow \prod_{i \geq 0} H^{2 i}(X, \mathbf{Q}) \\
& c h: K^{1}(X) \otimes \mathbf{Q} \rightarrow \prod_{i \geq 0} H^{2 i+1}(X, \mathbf{Q}) .
\end{aligned}
$$

These are isomorphisms for $X$ finite dimensional, and they can be used to prove that (1.0.1) $\otimes \mathbf{Q}$ degenerates in this case. An alternate proof is to use the Adams operations

$$
\Psi^{k}: K^{i}(X) \rightarrow K^{i}(X)
$$

One shows that $\Psi^{k}$ acts on (1.0.1) and induces multiplication by $q^{k}$ on $E_{2}^{p, 2 q}$. Hence all differentials in (1.0.1) are torsion, with explicit upper bounds; this yields in particular the formula: 


$$
K^{i}(X)^{(j)} \simeq H^{2 j+i}(X, \mathbf{Z}) \quad \text { up to groups of finite exponent }
$$

where $K^{i}(X)^{(j)}$ stands for the common eigenspace of weight $j$ on $K^{i}(X)$ for all Adams operations.

If $X$ is a finite $C W$-complex, its singular cohomology $H^{*}(X, \mathbf{Z})$ is finitely generated. This can be proven easily by induction on the number of cells, using the well-known cohomology of spheres:

$$
H^{i}\left(S^{n}, \mathbf{Z}\right)= \begin{cases}\mathbf{Z} & \text { if } i=0, n(\text { except } i=n=0 !) \\ 0 & \text { otherwise }\end{cases}
$$

By (1.0.1), this implies that the groups $K^{i}(X)$ are also finitely generated in this case. Conversely, suppose that for a given space $X$ we know that the $K^{i}(X)$ are finitely generated. Then using the partial degeneration of (1.0.1), we can deduce that $H^{*}(X, \mathbf{Z})$ is finitely generated up to some small torsion. This approach will fail if we want to get finite generation of $H^{*}(X, \mathbf{Z})$ on the nose, unless $X$ has small cohomological dimension.

\section{The picture in algebraic geometry}

In algebraic geometry, the picture is a bit more complicated.

\subsection{Algebraic $K$-theory}

Historically, the theory that was defined first was algebraic $K$-theory, denoted with lower indices. The definition of $K_{0}(X)$ ( $X$ a scheme) goes back to Grothendieck (late fifties) and actually predates topological $K$-theory: $K_{0}(X)$ classifies algebraic vector bundles over $X$, the relations being given by short exact sequences. Among the many proposed definitions for the higher $K$ groups, the one that was the most useful was that of Quillen around 1971/72 [153]: to any noetherian scheme he associates the category $\mathcal{M}(X)$ of coherent sheaves on $X$ and the full subcategory $\mathcal{P}(X)$ of locally free sheaves: $\mathcal{M}(X)$ is abelian and $\mathcal{P}(X)$ is an exact subcategory. Then

$$
\begin{aligned}
& K_{i}(X)=\pi_{i}(\Omega B Q \mathcal{P}(X)) \\
& K_{i}^{\prime}(X)=\pi_{i}(\Omega B Q \mathcal{M}(X))
\end{aligned}
$$

where $Q \mathcal{E}$ is Quillen's $Q$-construction on an exact category $\mathcal{E}$ and $B$ denotes the classifying space (or nerve) construction. For $i=0, K_{0}^{\prime}(X)$ classifies coherent sheaves on $X$ with respect to short exact sequences, a definition which also goes back to Grothendieck. There is always a map

$$
K_{*}(X) \rightarrow K_{*}^{\prime}(X)
$$

which is an isomorphism when $X$ is regular ("Poincaré duality"). 
Two fundamental additions to the foundations of algebraic $K$-theory were the works of Waldhausen in the early eighties [207] and Thomason-Trobaugh in the late eighties [195]. In particular, Thomason-Trobaugh slightly modifies the definition of Quillen's algebraic $K$-theory so as to obtain functoriality missing in Quillen's definition. His $K$-groups will be denoted here by $K^{T T}$ to distinguish them from those of Quillen: there is always a map

$$
K_{*}(X) \rightarrow K_{*}^{T T}(X)
$$

and this map is an isomorphism as soon as $X$ has an ample family of vector bundles, for example if $X$ is quasi-projective over an affine base, or is regular.

On the other hand, motivated by Matsumoto's theorem giving a presentation of $K_{2}$ of a field $k$ [129], Milnor introduced in [140] a graded commutative ring

$$
K_{*}^{M}(k)=T\left(k^{*}\right) / I
$$

where $T\left(k^{*}\right)$ is the tensor algebra of the multiplicative group of $k$ and $I$ is the two-sided ideal generated by the $x \otimes(1-x)$ for $x \neq 0,1$; its graded pieces are called the Milnor $K$-groups of $k$. Since algebraic $K$-theory has a product structure, there are natural homomorphisms

$$
K_{i}^{M}(k) \rightarrow K_{i}(k)
$$

which are isomorphisms for $i \leq 2$ (the case $i=2$ being Matsumoto's theorem) but not for $i \geq 3$. While Milnor stresses that his definition is purely ad hoc, it came as a surprise to many mathematicians that Milnor's $K$-groups are in fact not ad hoc at all and are fundamental objects in our story. See Theorem 2.2 .1 below as a sample.

We might say that algebraic $K$-theory is an algebro-geometric analogue of complex $K$-theory. It took about 10 more years to get a correct definition of the corresponding analogue of singular cohomology for smooth schemes over a field, and a further 6 or 7 years for arbitrary schemes over a field of characteristic 0. See the beautiful introduction of [14].

However, early on, Quillen already looked for a strengthening of this analogue and conjectured the following version of an Atiyah-Hirzebruch spectral sequence:

Quillen conjecture 2.1.1 ([155]). Let $A$ be a finitely generated regular Z-algebra of Krull dimension $d$ and $l$ a prime number. Then there exists a spectral sequence with $E_{2}$-terms

$$
E_{2}^{p, q}= \begin{cases}0 & \text { if } q \text { is odd } \\ H_{\text {ét }}^{p}\left(A\left[l^{-1}\right], \mathbf{Z}_{l}(i)\right) & \text { if } q=-2 i\end{cases}
$$

and whose abutment is isomorphic to $K_{-p-q}(A) \otimes \mathbf{Z}_{l}$ at least for $-p-q \geq 1+d$.

In this conjecture, the étale cohomology groups may be defined as inverse limits of étale cohomology groups with finite coefficients; they coincide 
with the continuous étale cohomology groups of Dwyer-Friedlander [44] and Jannsen [76] by Deligne's finiteness theorems [SGA 4 1/2, Th. finitude]. Note that if $A$ is a ring of integers of a number field, then such a spectral sequence must degenerate for cohomological dimension reasons when $l$ is odd or $A$ is totally imaginary, as pointed out by Quillen.

The Quillen conjecture and a complementary conjecture of Lichtenbaum relating algebraic $K$-theory and the Dedekind zeta function when $A$ is the ring of integers of a number field (see Conjecture 6.4.1 below) have inspired much of the development of the arithmetico-geometric side of algebraic $K$-theory ever since. For the benefit of the reader, let us shortly describe the progress towards this still unproven conjecture:

1. In [154] and [66], Quillen proved that the $K$-groups of rings of algebraic integers or of smooth curves over a finite field are finitely generated.

2. In [172], following a suggestion of Quillen, Soulé constructed higher Chern classes from algebraic $K$-theory with finite coefficients to étale cohomology. He proved that the corresponding $l$-adic homomorphisms are surjective up to finite torsion in the case of rings of integers in a global field. This gave the first application of algebraic $K$-theory to an algebraic problem outside the field (finiteness of certain $l$-adic cohomology groups).

3. In [48] and [49], Friedlander introduced étale $K$-theory in the geometric case, inspired by ideas from Artin-Mazur's étale homotopy (compare [8, p. 0.5]). He formulated a conjecture in [49] for complex varieties, related to Quillen's conjecture 2.1.1 and that he christened the Quillen-Lichtenbaum conjecture.

4. In [44], Dwyer and Friedlander defined continuous étale cohomology and [continuous] étale $K$-theory in full generality. They then did two quite different things:

a) They constructed a spectral sequence with $E_{2}$-terms the former, converging to the latter, for any $\mathbf{Z}\left[l^{-1}\right]$-scheme of finite étale cohomological $l$-dimension.

b) They defined a natural transformation

$$
K_{i}(X) \otimes \mathbf{Z}_{l} \rightarrow K_{i}^{\text {ét }}(X)^{\hat{n}}
$$

(where the right hand side is $l$-adic étale $K$-theory) and proved that this map is surjective when $X$ is the spectrum of a ring of integers of a global field.

This last result refined the result of Soulé, because in this case the spectral sequence of a) degenerates for dimension reasons. They reinterpreted Quillen's conjecture by conjecturing that the version of (2.1.1) with finite coefficients is an isomorphism for $i$ large enough, and christened this the "Lichtenbaum-Quillen conjecture".

5. In [43], Dwyer, Friedlander, Snaith and Thomason introduced algebraic $K$-theory with the Bott element inverted, proved that it maps to a version of étale $K$-theory and that, under some technical assumptions, this map 
is surjective. So "algebraic $K$-theory eventually surjects onto étale $K$ theory". (To the best of my knowledge, one can trace back the idea of using roots of unity to define an algebraic version of the Bott element, and to invert it, to Snaith [171].)

6. In [192], Thomason went a step further by showing that, at least for nice enough schemes, étale $K$-theory is obtained from algebraic $K$-theory by "inverting the Bott element". Hence, in this case, the spectral sequence of 4 a) converges to something close to algebraic $K$-theory. This refined the Dwyer-Friedlander result a). (Actually, Thomason constructs a priori a spectral sequence like that one converging to $K$-theory with the Bott element inverted, and uses it to show that this coincides with DwyerFriedlander's étale $K$-theory.)

7. Meanwhile, the Milnor conjecture and the more general Bloch-Kato conjecture (see Conjecture 4.2.3 below) showed up. The latter was proven in degree 2 by Merkurjev-Suslin [131]: this was the second application of algebraic $K$-theory to an algebraic problem outside algebraic $K$-theory (structure of central simple algebras). Then it was proven in degree 3 for $l=2$ by Merkurjev-Suslin [133] and independently Rost [160].

8. At the same time, Merkurjev-Suslin [132] and Levine [113] independently studied the indecomposable part of $K_{3}$ of a field $F$ (i.e. $K_{3}(F) / K_{3}^{M}(F)$ ). This was the first instance of work in the direction "the Bloch-Kato conjecture implies the Beilinson-Lichtenbaum conjecture".

9. In [114], Levine went a step further and proved that a form of the BlochKato conjecture for (possibly singular) semi-local rings implies a form of the Quillen-Lichtenbaum conjecture, expressed in terms of Soulé's higher Chern classes.

10. In [83] and [85], Kahn introduced anti-Chern classes going from étale cohomology to algebraic $K$-theory and étale $K$-theory, defined when the BlochKato conjecture is true; he recovered the results of Dwyer-Friedlander in this way.

11. In [74] (unfortunately unpublished), Hoobler proved that the Bloch-Kato conjecture for regular semi-local rings implies the same conjecture for arbitrary semi-local rings. A previous argument of Lichtenbaum [125], relying on Gersten's conjecture, showed that the Bloch-Kato conjecture for regular semi-local rings of geometric origin follows from the Bloch-Kato conjecture for fields.

12. Meanwhile, motivic cohomology started being introduced, first by Bloch and then by Friedlander, Suslin and Voevodsky. Spectral sequences from motivic cohomology to algebraic $K$-theory were constructed by BlochLichtenbaum [22], Friedlander-Suslin [51] and Levine [119], and with different ideas by Grayson [67] and Hopkins-Morel [75].

13. In [186], Suslin and Voevodsky formulated a Beilinson-Lichtenbaum conjecture for motivic cohomology (see Conjecture 4.2.4 below) and proved that, under resolution of singularities, it follows from the Bloch-Kato conjecture. In [61] and [62], Geisser and Levine removed the resolution of 
singularities assumption and also covered the case where the coefficients are a power of the characteristic.

14. Voevodsky proved the Bloch-Kato conjecture at the prime 2 [203] and conditionally at any prime [205].

15. Following this, the Quillen-Lichtenbaum conjecture at the prime 2 was proven by various authors $[159,88,91,121,151]$. Conditionally, the same proofs work at an odd prime (and are in fact simpler). If one had finite generation results for motivic cohomology, Conjecture 2.1.1 would follow from all this work.

Ironically, Thomason strongly disbelieved the Bloch-Kato conjecture [194, p. 409], while it was the key result that led to proofs of the QuillenLichtenbaum conjecture!

This concludes our short and necessarily incomplete survey. More details on parts of it will be found in the next sections.

\subsection{Bloch's cycle complexes}

See $\S 2$ and Appendix in Geisser's chapter, $\S \S 7,8$ in Grayson's chapter and $\S 2.3$ in Levine's chapter for more details in this subsection.

For $X$ a quasi-projective scheme over a field $k$, Bloch introduced the cycle complexes in 1984 [20]. Denote by $\Delta \bullet$ the standard cosimplicial scheme over $k$ with

$$
\Delta^{p}=\operatorname{Spec} k\left[t_{0}, \ldots, t_{p}\right] /\left(\sum t_{i}-1\right) .
$$

We define the homological cycle complex of dimension $n$ of $X$ as the chain complex $z_{n}(X, *)$ associated to the simplicial abelian group $z_{n}(X, \bullet)$, where, for all $p, z_{n}(X, p)$ is the group of cycles of dimension $n+p$ on $X \times \Delta^{p}$ meeting all faces properly; the faces and degeneracies are induced by those of $\Delta^{\bullet}$. The (co)homology groups of $z_{n}(X, *)$ are called the higher Chow groups of $X$ :

$$
C H_{n}(X, p)=H_{p}\left(z_{n}(X, *)\right) .
$$

These groups are 0 for $p<0$ or $n+p<0$ by dimension reasons. Beyond these trivial examples, let us give two other characteristic ones. First, for $p=0$, one recovers the classical Chow group $C H_{n}(X)$, as is easily seen: this justifies the terminology. Less easy is the following theorem, due independently to Nesterenko-Suslin and Totaro, when $X=\operatorname{Spec} k$ for $k$ a field.

Theorem 2.2.1 ([148, 196]). $C H_{-n}(k, n) \simeq K_{n}^{M}(k)$.

Higher Chow groups form, not a cohomology theory, but a Borel-Moore homology theory on $k$-schemes of finite type. For example, if $Z$ is a closed subset of $X$ with open complement $U$, then one has a long localisation exact sequence $[20,21]$

$$
\cdots \rightarrow C H_{p}(Z, n) \rightarrow C H_{p}(X, n) \rightarrow C H_{p}(U, n) \rightarrow C H_{p-1}(Z, n) \rightarrow \ldots
$$


This is a hard theorem. Using it, one derives Mayer-Vietoris long exact sequences for open covers, which allows one to extend the definition of higher Chow groups to arbitrary $k$-schemes $X$ essentially of finite type by the formula

$$
C H_{p}(X, n)=\mathbb{H}_{\mathrm{Zar}}^{-p}\left(X, \underline{z}_{n}(-, *)\right)
$$

where $\underline{z}_{n}(-, *)$ is the sheaf of complexes associated to the presheaf $U \mapsto$ $z_{n}(U, *)$.

Even harder is the "Atiyah-Hirzebruch" spectral sequence (Bloch-Lichtenbaum [22], Friedlander-Suslin [51], Levine [119, 122])

$$
E_{p, q}^{2}=H_{p}^{B M}(X, \mathbf{Z}(-q / 2)) \Rightarrow K_{p+q}^{\prime}(X)
$$

where we have renumbered the higher Chow groups by the rule

$$
H_{p}^{B M}(X, \mathbf{Z}(n)):=C H_{n}(X, p-2 n)
$$

and $H_{p}^{B M}(X, \mathbf{Z}(-q / 2))$ is defined to be 0 if $q$ is odd.

If $X$ is smooth of pure dimension $d$, we again change the notation by setting

$$
\begin{aligned}
C H^{n}(X, p) & :=C H_{d-n}(X, p) \\
H^{p}(X, \mathbf{Z}(n)) & :=C H^{n}(X, 2 n-p)=H_{2 d-p}^{B M}(X, \mathbf{Z}(d-n)) .
\end{aligned}
$$

We then extend this definition by additivity to the case where $X$ is not equidimensional. Given the isomorphism $K_{*}(X) \stackrel{\sim}{\longrightarrow} K_{*}^{\prime}(X)$, the spectral sequence (2.2.2) may now be rewritten

$$
E_{2}^{p, q}=H^{p}(X, \mathbf{Z}(-q / 2)) \Rightarrow K_{-p-q}(X)
$$

resembling (1.0.1) closely. For future reference, let us record the mod $n$ version of this spectral sequence:

$$
E_{2}^{p, q}=H^{p}(X, \mathbf{Z} / n(-q / 2)) \Rightarrow K_{-p-q}(X, \mathbf{Z} / n) .
$$

Rather easier to prove are the Chern character isomorphisms (Bloch [20], Levine $[116,117])$

$$
\begin{aligned}
& K_{i}^{\prime}(X) \otimes \mathbf{Q} \stackrel{\sim}{\longrightarrow} \bigoplus_{n \in \mathbf{Z}} H_{2 n-i}^{B M}(X, \mathbf{Q}(n)) \\
& K_{i}(X) \otimes \mathbf{Q} \stackrel{\sim}{\longrightarrow} \bigoplus_{n \in \mathbf{Z}} H^{2 n-i}(X, \mathbf{Q}(n)) \quad(X \text { smooth }) .
\end{aligned}
$$

They might be used to prove the degeneration of (2.2.2) and (2.2.3) up to small torsion, although I don't think this has been done yet. However, one may use Adams operations for this, just as in topology (Soulé [177], Gillet-Soulé $[65, \S 7])$, which yields the formula analogous to (1.0.2): 


$$
\begin{aligned}
& K_{i}(X)^{(j)} \simeq H^{2 j-i}(X, \mathbf{Z}(i)) \quad(X \text { smooth }) \\
& K_{i}^{\prime}(X)_{(j)} \simeq H_{2 j+i}^{B M}(X, \mathbf{Z}(i))
\end{aligned}
$$

up to groups of finite exponent, where $K_{i}^{\prime}(X)_{(j)}$ are the eigenspaces of the homological Adams operations [176, Th. 7 p. 533].

The above picture may be extended to schemes of finite type (resp. regular of finite type) over a Dedekind scheme (Levine [119, 121], see also Geisser [59] and $\S 3.3$ of Geisser's chapter in this Handbook).

Remark 2.2.2. Let $f: X \rightarrow Y$ be a morphism of schemes, taken smooth over a field to fix ideas. Suppose that $f$ induces an isomorphism on motivic cohomology groups. Then the spectral sequence (2.2.3) shows that $f$ also induces an isomorphism on $K$-groups. By $(2.2 .7)$, the converse is true up to small torsion, but I doubt that it is true on the nose, except in small-dimensional cases. The situation is quite similar to that in Thomason's proof of absolute cohomological purity for étale cohomology with torsion coefficients [193]: Thomason's proof gave the result only up to small torsion, and it required delicate further work by Gabber to get the theorem in its full precision (see Fujiwara's exposition of Gabber's proof in [53]).

\subsection{Suslin-Voevodsky's motivic cohomology}

See $§ 2.4$ in Levine's chapter and also Friedlander's Bourbaki talk [50] for more details on this subsection.

Of course, defining a cohomology theory on smooth schemes as a renumbering of a Borel-Moore homology theory is a kind of a cheat, and it does not define motivic cohomology for non-smooth varieties. Friedlander, Suslin and Voevodsky $(1987-1997,[185,52,198,186])$ have associated to any scheme of finite type $X$ over a field $k$ a motivic complex of sheaves $C_{*}(X)=C_{*}(L(X))$ on the category $S m / k$ of smooth $k$-varieties provided with the Nisnevich topology, where, for $U \in S m / k, L(X)(U)$ is the free abelian group with basis the closed integral subschemes of $X \times U$ which are finite and surjective over a component of $U$, and for a presheaf $\mathcal{F}, C_{*}(\mathcal{F})$ is the complex of presheaves with $C_{n}(\mathcal{F})$ defined by

$$
C_{n}(\mathcal{F})(U)=\mathcal{F}\left(U \times \Delta^{n}\right)
$$

Then they define for each $n$ a sheaf $L\left(\mathbb{G}_{m}^{\wedge n}\right)$ as the cokernel of the map

$$
\bigoplus_{i=1}^{n-1} L\left(\mathbb{G}_{m}^{n-1}\right) \rightarrow L\left(\mathbb{G}_{m}^{n}\right)
$$

induced by the embeddings of the form

$$
\left(x_{1}, \ldots, x_{n-1}\right) \mapsto\left(x_{1}, \ldots, 1, \ldots, x_{n-1}\right)
$$


(it is in fact a direct summand). Finally, they set $\mathbf{Z}_{S V}(n):=C_{*}\left(L\left(\mathbb{G}_{m}^{\wedge n}\right)\right)[-n]$ (the index SV is ours, in order to avoid notational confusion).

If $X$ is smooth, then there are canonical isomorphisms [200]

$$
H^{i}(X, \mathbf{Z}(n)) \simeq \mathbb{H}_{\text {Nis }}^{i}\left(X, \mathbf{Z}_{S V}(n)\right) .
$$

However, in general one does not expect the right-hand-side group to have good functorial properties. For this, one has to replace the Nisnevich topology by the stronger cdh topology. If $\operatorname{char} k=0$, resolution of singularities implies that, for $X$ smooth, the natural maps

$$
H_{\mathrm{Nis}}^{i}\left(X, \mathbf{Z}_{S V}(n)\right) \rightarrow \mathbb{H}_{c d h}^{i}\left(X, \mathbf{Z}_{S V}(n)\right)
$$

are isomorphisms [198]. However this is not known in characteristic $p$. One may therefore say that the Suslin-Voevodsky approach yields the "correct" motivic cohomology for all schemes in characteristic 0 , but does so only conjecturally in characteristic $p$, because (unlike Bloch's approach) it relies fundamentally on resolution of singularities. For this reason and others, we shall mainly work with Bloch's cycle complexes in the sequel.

Using these ideas, Suslin has recently proven that a spectral sequence constructed by Grayson [67] based on ideas of Goodwillie and Lichtenbaum and converging to algebraic $K$-theory for $X$ smooth semi-local essentially of finite type over a field has its $E_{2}$-terms isomorphic to motivic cohomology [184]. Thus we get a spectral sequence like (2.2.3), independent of the Bloch-Lichtenbaum construction. It is not clear, however, that the two spectral sequences coincide.

One cannot expect a spectral sequence like (2.2.3) for arbitrary schemes of finite type, even over a field of characteristic 0 , nor Chern character isomorphisms as (2.2.6). Indeed, motivic cohomology is homotopy invariant while algebraic $K$-theory is not. One can however expect that (2.2.3) and (2.2.6) generalise to arbitrary schemes of finite type $X$ by replacing algebraic $K$-theory by Weibel's homotopy invariant algebraic $K$-theory $K H(X)$ [209]. This has been done recently in characteristic 0 by Christian Haesemeyer, who produced a spectral sequence (reproduced here up to the indexing)

$$
H^{p}(X, \mathbf{Z}(-q / 2)) \Rightarrow K H_{-p-q}(X)
$$

for $X$ of finite type over a field of characteristic 0 [70, Th. 7.11]. This goes some way towards the following general conjecture:

Conjecture 2.3.1 ( $c f$. Beilinson [12, 5.10 B and C (vi)]). Let $m \geq 1$ and let $X$ be a Noetherian separated $\mathbf{Z}[1 / m]$-scheme of finite Krull dimension. Then there is a spectral sequence

$$
E_{2}^{p, q}=H_{\mathrm{Zar}}^{p}(X, B / m(-q / 2)) \Rightarrow K_{-p-q}^{T T}(X, \mathbf{Z} / m)
$$

degenerating up to small torsion. 
Here $B / m(n)=\tau_{\leq n} R \alpha_{*} \mu_{m}^{\otimes n}$ where $\alpha$ is the projection of the big étale site of $\operatorname{Spec} \mathbf{Z}[1 / m]$ onto its big Zariski site, and $K^{T T}$ is Thomason-Trobaugh $K$-theory, $c f$. 2.1. (Note that $K_{*}^{T T}(X, \mathbf{Z} / m)$ is homotopy invariant: Weibel [208, Cons. 1.1], Thomason [195, Th. 9.5 a)].) See Corollary 4.2.9 below for an explanation of this conjecture, and Theorem 5.0.8 for evidence to it.

\subsection{The Beilinson-Soulé conjecture}

As a basic difference between algebraic topology and algebraic geometry, the analogue of the following conjecture is trivially true for singular cohomology:

Beilinson-Soulé conjecture 2.4.1 ([10], [176, Conj. p. 501]). For $X$ regular, $H^{i}(X, \mathbf{Z}(n))=0$ for $n \geq 0$ and $i<0$ (even for $i=0$ when $n>0$ ).

This conjecture is central in the developments of the theory of motives, and we shall come back to it in this survey every now and then.

Let us toy with the Beilinson-Soulé conjecture a little. Let $Z$ be a regular closed subset of $X$ of codimension $c$ and $U$ be the open complement. The Gysin exact sequence for motivic cohomology (an equivalent form of (2.2.1)) reads

$$
\begin{aligned}
\cdots \rightarrow H^{i-2 c}(Z, \mathbf{Z}(n-c)) \rightarrow H^{i}(X, \mathbf{Z}(n)) \rightarrow H^{i}(U, \mathbf{Z}(n)) \\
\rightarrow H^{i-2 c+1}(Z, \mathbf{Z}(n-c)) \rightarrow \ldots
\end{aligned}
$$

Suppose we have found an inductive proof of the conjecture: induction could be either on $n$ or on $\operatorname{dim} X$, or on both. In each case we find inductively that the map $H^{i}(X, \mathbf{Z}(n)) \rightarrow H^{i}(U, \mathbf{Z}(n))$ is an isomorphism. On the other hand, motivic cohomology transforms filtering inverse limits of regular schemes with affine transition morphisms into direct limits. From this, one deduces easily:

Lemma 2.4.2. The following conditions are equivalent:

(i) The Beilinson-Soule conjecture is true.

(ii) The Beilinson-Soulé conjecture is true for all fields.

(iii) The Beilinson-Soulé conjecture is true for all finitely generated fields.

(iv) The Beilinson-Soulé conjecture is true for all regular schemes of finite type over $\operatorname{Spec} \mathbf{Z}$.

If one inputs Hironaka's resolution of singularities or de Jong's alteration theorems [82, Th. 4.1 and 8.2], one gets stronger results:

Lemma 2.4.3. a) If we restrict to regular schemes over $\mathbf{Q}$, the following condition is equivalent to those of the previous lemma:

(v) The Beilinson-Soulé conjecture is true for all smooth projective varieties over $\operatorname{Spec} \mathbf{Q}$. 
b) If we restrict to regular schemes over $\mathbf{F}_{p}$ and tensor groups with $\mathbf{Q}$, the following condition is equivalent to those of the previous lemma:

(vi) The Beilinson-Soulé conjecture is true for all smooth projective varieties over $\operatorname{Spec} \mathbf{F}_{p}$.

c) If we tensor groups with $\mathbf{Q}$, the following condition is equivalent to those of the previous lemma:

(vii) The Beilinson-Soulé conjecture is true for all regular projective schemes over $\operatorname{Spec} \mathbf{Z}$, generically smooth over a suitable ring of integers of a number field and with strict semi-stable reduction outside the smooth locus.

The dévissage arguments for this are standard and I shall only recall them sketchily: for more details see Geisser's survey article on applications of de Jong's theorem [57], or also [94]. There are two main steps:

1. Given $X$ regular and $Z \subset X$ closed regular with open complement $U$, the exact sequence (2.4.1) shows inductively that the conjecture is true for $X$ if and only if it is true for $U$. If $U$ is now any open subset of $X$, a suitable stratification of $X-U$ reduces us to the first case, in characteristic $p$ because finite fields are perfect and over $\mathbf{Z}$ by [EGA IV, cor. 6.12.6].

2. By Hironaka in characteristic 0 , any smooth variety $X$ contains an open subset $U$ which is an open subset of a smooth projective variety. By de Jong in characteristic $p$ (resp. over $\mathbf{Z}$ ), any regular $X$ contains an $U$ such that there exists a finite étale map $f: \tilde{U} \rightarrow U$ such that $\tilde{U}$ is contained in a smooth projective variety (resp. in a scheme as in (vii). A transfer argument finishes the proof (and uses coefficients $\mathbf{Q}$ ).

We shall see in Subsection 4.3 that tensoring groups by $\mathbf{Q}$ is not a very serious restriction by now.

The Beilinson-Soulé conjecture is true for $n=0$ because $\mathbf{Z}(0)=\mathbf{Z}$ and for $n=1$ because $\mathbf{Z}(1) \simeq \mathbb{G}_{m}[-1]$. It is also true for $X$ finitely generated over $\mathbf{Z}$ of Krull dimension $\leq 1$ (see Lemma 6.1.6), and for some smooth projective varieties over $\mathbf{F}_{p}$ (see Section 7). Although some mathematicians have doubted its validity in recent years, it is my belief that it is true in general and will be proven by arithmetic means, analytic means or a combination of both. Evidence for this will appear in Sections 6 and 7.

Finally, there is a companion conjecture to the Beilinson-Soulé conjecture. For fields, it was formulated in weight 2 by Merkurjev and Suslin [133, Conj. 11.7], and we take the liberty to attribute it to them in general. ${ }^{1}$

\footnotetext{
${ }^{1}$ In this we follow a well-established tradition in algebraic $K$-theory which consists in attributing conjectures to people who did not really formulate them in those terms. For example, Beilinson and Soulé did not actually formulate Conjecture 2.4.1 as it stands, because at the time motivic cohomology had not been defined. However they formulated it in terms of the gamma filtration on algebraic $K$ theory and Beilinson envisioned a motivic cohomology which would explain this
} 
Merkurjev-Suslin conjecture 2.4.4. For any regular scheme $X$, let $X_{0}$ be the "scheme of constants" of $X$, that is, the normalisation of Spec $\mathbf{Z}$ into $X$ (for example if $X=\operatorname{Spec} F$ with $F$ a field, then $X_{0}=\operatorname{Spec} F_{0}$ where $F_{0}$ is the algebraic closure in $F$ of its prime subfield). Then for all $n \geq 2$, the map $H^{1}\left(X_{0}, \mathbf{Z}(n)\right) \rightarrow H^{1}(X, \mathbf{Z}(n))$ is an isomorphism.

The same reductions as for the Beilinson-Soulé conjecture apply to this conjecture.

\section{Review of motives}

The aim of this section is to present a state-of-the-art description of the current understanding of the "theory of motives", whose idea is fully due to Grothendieck in the mid sixties (see [69]). This description will be very sketchy: a thorough one would go far beyond the scope of this survey.

For an overlapping but different and much more detailed survey, we invite the reader to consult Marc Levine's chapter in this Handbook.

We work over a base field $k$. We won't enter the description of categories of motives over other bases.

\subsection{Pure motives}

For more background on this subsection, we refer the reader to [128], [162, Ch. VI §4], [41], [108], [166] and [3].

To define a category of pure motives, one needs

1. a commutative ring $A$ of coefficients;

2. an "adequate" equivalence relation $\sim$ on algebraic cycles (on smooth projective varieties) with coefficients in $A$ : roughly, modulo $\sim$, direct and inverse images and intersection products can be defined.

We shall refer to a pair $(A, \sim)$ as above as to an adequate pair. For $X$ smooth projective, the groups of cycles on $X$ modulo $\sim$ will be denoted by $\mathcal{Z}_{\sim}^{*}(X, A)$.

The finest adequate equivalence relation is rational (= linear) equivalence rat, and when $A$ contains $\mathbf{Q}$, the coarsest one is numerical equivalence num. Between the two, one finds other interesting adequate equivalence relations (in increasing order of coarseness):

conjecture. Similarly, the Beilinson-Lichtenbaum conjecture 4.2.4 was formulated by Beilinson and Lichtenbaum as a conjecture about a yet conjectural motivic cohomology. One last example is the name "Quillen-Lichtenbaum" given to the conjecture asserting that algebraic and étale $K$-theories with finite coefficients should agree in large enough degrees, while étale $K$-theory had been neither invented at the time when they made the corresponding conjectures relating algebraic $K$-theory and étale cohomology, nor even envisioned by them! 
- algebraic equivalence;

- Voevodsky's smash-nilpotence equivalence [197] (the observation that it defines an adequate equivalence relation is due to Y. André);

- homological equivalence relative to a given Weil cohomology theory.

By definition, one has $\mathcal{Z}_{\text {rat }}^{*}(X, \mathbf{Z})=C H^{*}(X)$.

Now, given an adequate pair $(A, \sim)$, one first constructs the category of $(A, \sim)$-correspondences $\operatorname{Cor}_{\sim}(k, A)$. Objects are smooth projective $k$-varieties; for $X$ smooth projective we denote by $[X]$ its image in $\operatorname{Cor}_{\sim}(k, A)$. For $X, Y$ two smooth projective varieties one defines

$$
\operatorname{Hom}([X],[Y])=\mathcal{Z}_{\sim}^{\operatorname{dim} Y}(X \times Y, A) .
$$

Composition of correspondences $\alpha: X \rightarrow Y$ and $\beta: Y \rightarrow Z$ is given by the sempiternal formula

$$
\beta \circ \alpha=\left(p_{X Z}\right)_{*}\left(p_{X Y}^{*} \alpha \cdot p_{Y Z}^{*} \beta\right) .
$$

For a morphism $f: X \rightarrow Y$, denote by $\Gamma_{f}$ its graph, viewed as an algebraic cycle on $X \times Y$. Then $f \mapsto\left[\Gamma_{f}\right]$ defines a functor $\operatorname{SmProj}(k) \rightarrow \operatorname{Cor}_{\sim}(k, A)$. One should be careful that this functor is covariant here, which is the convention in Fulton [54] and Voevodsky [198] but is opposite to the point of view of Grothendieck and his school.

We may put on $\operatorname{Cor}_{\sim}(k, A)$ a symmetric monoidal structure by the rule $[X] \otimes[Y]=[X \times Y]$, the tensor product of correspondences being given by their external product. Then $\mathbf{1}=h(\operatorname{Spec} k)$ is the unit object of this structure.

Once the category of correspondences is defined, we get to the category of pure motives by two formal categorical operations:

- Effective pure motives: take the pseudo-abelian (karoubian) envelope of $\operatorname{Cor}_{\sim}(k, A)$. This amounts to formally adjoin kernels to idempotent endomorphisms. The resulting category $\operatorname{Mot}_{\sim}^{\mathrm{eff}}(k, A)$ is still monoidal symmetric. We write $h(X)$ for the image of $[X]$ in $\operatorname{Mot}_{\sim}^{\text {eff }}(k, A)$.

- Pure motives: in $\operatorname{Mot}_{\sim}^{\text {eff }}(k, A)$, we have a decomposition $h\left(\mathbf{P}^{1}\right)=\mathbf{1} \oplus L$ given by the choice of any rational point: $L$ is called the Lefschetz motive. Tensor product by $L$ is fully faithful. We may then formally invert $L$ for the monoidal structure: the resulting category $\operatorname{Mot}_{\sim}(k, A)$ inherits a symmetric monoidal structure because the permutation (123) acts on $L^{\otimes 3}$ by the identity (this necessary and sufficient condition which does not appear for instance in Saavedra [162] was first noticed by Voevodsky) ${ }^{2}$. Here too we shall depart from a more traditional notation by writing $M(n)$ for $M \otimes L^{\otimes n}$ : this object is usually written $M(-n)$ in the Grothendieck school.

\footnotetext{
${ }^{2}$ In fact we even have that the permutation (12) acts on $L^{\otimes 2}$ by the identity.
} 
The category $\operatorname{Mot}_{\sim}(k, A)$ is rigid: any object $M$ has a dual $M^{\vee}$ and any object is canonically isomorphic to its double dual. If $X$ has pure dimension $d$, then $h(X)^{\vee}=h(X)(-d)$ and the unit and counit of the duality are both given by the class of the diagonal in $\operatorname{Hom}\left(\mathbf{1}, h(X) \otimes h(X)^{\vee}\right) \simeq$ $\operatorname{Hom}\left(h(X)^{\vee} \otimes h(X), \mathbf{1}\right)=\mathcal{Z}_{\sim}^{d}(X \times X, A)$. All this theory is purely formal beyond the projective line formula:

$$
\mathcal{Z}_{\sim}^{n}\left(X \times \mathbf{P}^{1}, A\right)=\mathcal{Z}_{\sim}^{n}(X, A) \oplus \mathcal{Z}_{\sim}^{n-1}(X, A) .
$$

Then one is interested in finer properties of $\operatorname{Mot}_{\sim}(k, A)$. The most important result is Jannsen's theorem, which was for a long time a standard conjecture:

Theorem 3.1.1 (Jannsen [79]). The category $\operatorname{Mot}_{\sim}(k, \mathbf{Q})$ is semi-simple if and only if $\sim=$ num.

\section{What we don't know about pure motives}

What is missing from a rigid tensor category to be tannakian (hence to be classified by a gerbe) is a fibre functor (detailing these notions would go beyond the scope of this survey and we can only refer the reader to the excellent references [162], [39], [28]). Morally, such a fibre functor on $\operatorname{Mot}_{\text {num }}(k, \mathbf{Q})$ should be given by any Weil cohomology theory $H$. However there are two unsolved problems here:

- We don't know whether homological equivalence (relative to $H$ ) equals numerical equivalence.

- Even so, there is a more subtle problem that the category of pure motives as defined above is "false": it cannot be tannakian! Namely, let $\operatorname{dim} M$ be the "rigid dimension" of an object $M$, defined as the trace of $1_{M}$. In any tannakian category, the dimension is a nonnegative integer because it can be computed as the dimension of a vector space via a fibre functor. Now if $M=h(X)$, computing $\operatorname{dim} X$ via $H$ gives $\operatorname{dim} X=\chi(X)$, the EulerPoincaré characteristic of $X$, which can be any kind of integer (it is $2-2 g$ if $X$ is a curve of genus $g$ ).

The second problem is a matter of the commutativity constraint in $\operatorname{Mot}_{H}(k, \mathbf{Q})$. To solve it, one looks at the Künneth projectors, i.e. the projectors $\pi_{i}$ from $H^{*}(X)$ onto its direct summands $H^{i}(X)$. In case the $\pi_{i}$ are given by algebraic correspondences, one can use them to modify the commutativity constraint (by changing signs) and tranform the Euler-Poincaré characteristic into the sum of dimensions of the $H^{i}(X)$, which is certainly a nonnegative integer. To do this, it suffices that the sum of the $\pi_{2 i}$ be algebraic.

The two conjectures:

(HN) Homological and numerical equivalences coincide

(C) For any $X$ and $H$, the Künneth projectors are algebraic 
are part of Grothendieck's standard conjectures ${ }^{3}$ [68]. This is not the place to elaborate on them: see also [107], [109], [162, p. 380] and [2, §1 and Appendix] for more details. Let us just mention that there is another conjecture B (the "Lefschetz-type conjecture") and that

\section{Conjecture $\mathrm{HN} \Rightarrow$ Conjecture $\mathrm{B} \Rightarrow$ Conjecture $\mathrm{C}$}

where the first implication is an equivalence in characteristic 0 .

Under these conjectures, one can modify $\operatorname{Mot}_{\text {num }}(k, \mathbf{Q})$ and make it a tannakian category, semi-simple by Theorem 3.1.1. To the fibre functor $H$ will then correspond its "motivic Galois group" (ibid.), a proreductive group defined over the field of coefficients of $H$, that one can then use to do "motivic Galois theory". Moreover the Künneth components yield a weight grading on $\operatorname{Mot}_{\text {num }}(k, \mathbf{Q})$.

Lieberman [127] proved Conjecture B for abelian varieties (over C; see Kleiman [107] for a write-up over an arbitrary field), and Katz-Messing [104] proved Conjecture $\mathrm{C}$ when $k$ is finite for $l$-adic and crystalline cohomology, as a consequence of Deligne's proof of the Weil conjecture (Riemann hypothesis) [34]. Besides these special cases and a few more easy cases, these conjectures are still open now.

Suppose that $k$ is finite, and take $H=H_{l}, l$-adic cohomology (where $l$ is a prime number different from char $k$ ). Still by Katz-Messing, for any $X$ the ideal

$$
\operatorname{Ker}\left(\operatorname{End}_{\mathrm{Mot}_{H}}(h(X)) \rightarrow \operatorname{End}_{\text {Mot }_{\text {num }}}(h(X))\right)
$$

is nilpotent: this implies that the functor $\operatorname{Mot}_{H}(k, \mathbf{Q}) \rightarrow \operatorname{Mot}_{\text {num }}(k, \mathbf{Q})$ is essentially surjective. It follows that one can "push down" from $\operatorname{Mot}_{H}(k, \mathbf{Q})$ to $\operatorname{Mot}_{\text {num }}(k, \mathbf{Q})$ the change of commutativity constraint. Then the new $\otimes-$ category $\widetilde{\operatorname{Mot}}_{\text {num }}(k, \mathbf{Q})$ is semi-simple rigid and any object has a nonnegative dimension: a theorem of Deligne [37] then implies that it is (abstractly) tannakian. For details, see Jannsen [79].

Finally we should mention Voevodsky's conjecture:

Conjecture 3.1.2 ([197]). Smash-nilpotence and numerical equivalence coincide.

It is stronger than the first standard conjecture above, and has the advantage not to single out any Weil cohomology.

\section{Getting around the standard conjectures}

There are two ways to make the approach above both more unconditional and more explicit. The first one was initiated by Deligne in [35] using (in characteristic 0) the notion of an absolute Hodge cycle on a smooth projective

\footnotetext{
3 The terminology "standard conjectures" is not limited to Grothendieck: Serre used it in [169] with a quite different, although closely related, meaning.
} 
variety $X$, which is a system of cohomology classes in all "classical" cohomology theories applied to $X$, corresponding to each other via the comparison isomorphisms: the classes of a given algebraic cycle define an absolute Hodge cycle and the Hodge conjecture asserts that there are no others. This approach was refined and made almost algebraic by Yves André in [2]. Like Deligne, André's idea is to adjoin cycles that one hopes eventually to be algebraic: but he just takes the inverses of the Lefschetz operators in the graded cohomology ring $H^{*}(X)$ (for some classical Weil cohomology $H$ ) and shows that, if char $k=0$, he gets a semi-simple tannakian category (with fibre functor given by $H$ ), a priori with larger Hom groups than $\operatorname{Mot}_{H}(k, \mathbf{Q})$.

The second and rather opposite approach, due to André and the author [5], consists of restricting a priori to the full $\otimes$-subcategory $\operatorname{Mot}_{H}^{+}(k, \mathbf{Q})$ formed of those homological motives whose even Künneth projectors are algebraic. After showing that its image $\operatorname{Mot}_{\text {num }}^{+}(k, \mathbf{Q})$ in $\operatorname{Mot}_{\text {num }}(k, \mathbf{Q})$ does not depend on the choice of a "classical" Weil cohomology $H,{ }^{4}$ we show that the projection functor $\operatorname{Mot}_{H}^{+}(k, \mathbf{Q}) \rightarrow \operatorname{Mot}_{\text {num }}^{+}(k, \mathbf{Q})$ has monoidal sections, unique up to monoidal conjugation: this depends on the results of [6]. Then $H$ defines an essentially unique fibre functor on $\operatorname{Mot}_{\text {num }}^{+}(k, \mathbf{Q})$ after the suitable modification of the commutativity constraints.

\subsection{The conjectural abelian category of mixed motives}

See $\S 3$ in Levine's chapter for more details on this subsection.

What about varieties $X$ that are not smooth projective? Elementary cases show that their cohomology, viewed in enriched categories, is not in general semi-simple. For example, the $l$-adic cohomology of $X$ is not in general semisimple as a $G_{k}$ representation. Or, if $k=\mathbf{C}$, the Betti cohomology of $X$ is not in general a semi-simple mixed Hodge structure.

Therefore one cannot expect that general varieties are classified by a semisimple tannakian category. One still hopes, however, that they are classified by a (not semi-simple) tannakian category $\operatorname{MMot}(k, \mathbf{Q})$ : see especially $[12$, 5.10]. Here are some conjectural properties of this conjectural category (the list is not exhaustive of course):

- $\operatorname{MMot}(k, \mathbf{Q})$ is tannakian and every object has finite length.

- The socle of $\operatorname{MMot}(k, \mathbf{Q})$ (i.e. the full subcategory of semisimple objects) is $\operatorname{Mot}_{\text {num }}(k, \mathbf{Q})$.

- There is a weight filtration on $\operatorname{MMot}(k, \mathbf{Q})$ which extends the weight grading of $\operatorname{Mot}_{\text {num }}(k, \mathbf{Q})$; its associated graded (for any object) belongs to $\operatorname{Mot}_{\text {num }}(k, \mathbf{Q})$.

- Any variety $X$ has cohomology objects and cohomology objects with compact supports $h^{i}(X), h_{c}^{i}(X) \in \operatorname{MMot}(k, \mathbf{Q})$, with Künneth formulas; $h^{*}$ is contravariant for all morphisms while $h_{c}^{*}$ is contravariant for proper

\footnotetext{
${ }^{4}$ If char $k=0$ this is obvious via the comparison theorems; in positive characteristic it depends on the Weil conjectures (Riemann hypothesis).
} 
morphisms. There are canonical morphisms $h_{c}^{i}(X) \rightarrow h^{i}(X)$ which are isomorphisms for $X$ proper.

- There are blow-up exact sequences for $h^{*}$, localisation exact sequences for $h_{c}^{*}$ and Mayer-Vietoris exact sequences for both.

- For any $X$, the natural maps $h^{*}(X) \rightarrow h^{*}\left(X \times \mathbf{A}^{1}\right)$ are isomorphisms.

- If $X$ is smooth of pure dimension $d$, one has canonical isomorphisms $h_{c}^{i}(X)^{\vee} \simeq h^{2 d-i}(X)(-d)$.

- For all $X, n$ with $X$ smooth there is a spectral sequence $\operatorname{Ext}_{\text {MMot }}^{p}\left(\mathbf{1}, h^{q}(X)(n)\right) \Rightarrow H^{p+q}(X, \mathbf{Q}(n))$; if $X$ is smooth projective, it degenerates up to torsion and yields, for $p+q=2 n$, the famous Bloch-Beilinson-Murre filtration on Chow groups, $c f$. [12, 5.10 C]. (For more details on this filtration, see Jannsen [81]).

Note that the last property contains the Beilinson-Soule conjecture 2.4.1, since $\operatorname{Ext}^{p}=0$ for $p<0$. See Levine's chapter, Conjecture 3.4, for a slightly different set of properties.

\subsection{The nonconjectural triangulated categories of mixed motives}

One expects that $\operatorname{MMot}(k, \mathbf{Q})$ will arise as the heart of a $t$-structure on a tensor triangulated category. There are currently 3 constructions of such a category:

- Hanamura's construction [71], [72];

- Levine's construction [118];

- Voevodsky's construction [198].

For a discussion of the constructions of these categories and their comparisons, we refer the reader to $\S 4$ in Marc Levine's chapter. Here are briefly some of their common features (for simplicity, let us write $D(k)$ for any of these categories):

1. $D(k)$ is a $\mathbf{Z}$-linear tensor triangulated category; it is rigid if $\operatorname{char} k=0$, or after tensoring with $\mathbf{Q}$ in characteristic $p$ (Hanamura's category has rational coefficients anyway).

2. There is a canonical fully faithful tensor functor

$$
\delta: \operatorname{Mot}_{\text {rat }}(k, \mathbf{Z}) \rightarrow D(k)
$$

(for Hanamura, tensor with $\mathbf{Q}$ ). The image of $L$ under this functor is denoted by $\mathbf{Z}(1)[2]$ by Voevodsky. (Note that Voevodsky calls $\mathbf{Z}(1)$ the "Tate object", so that $\delta$ sends the Lefschetz motive to a shift of the Tate object!)

3. Any smooth variety $X$ (smooth projective for Hanamura) has a "motive" $M(X) \in D(k)$, which is contravariant in $X$ in Levine and Hanamura, covariant in Voevodsky; there are Mayer-Vietoris exact triangles (for open covers) and $M(X)$ is homotopy invariant. If char $k=0$, any variety has 
a motive $M(X)$ and a Borel-Moore motive $M^{c}(X)$; on top of the above properties, there are blow-up and localisation exact triangles. There is a canonical morphism $M(X) \rightarrow M^{c}(X)$ which is an isomorphism when $X$ is proper.

4. If $X$ is smooth of pure dimension $d$, there is a "Poincare duality" isomorphism $M^{c}(X) \simeq M(X)^{\vee}(d)[2 d]$.

5. For any $X$ (smooth in characteristic $p$ ) one has canonical isomorphisms $\operatorname{Hom}\left(\mathbf{Z}(p)[q], M^{c}(X)\right)=H_{q}^{B M}(X, \mathbf{Z}(p))$. For $X$ smooth, one has canonical isomorphisms $\operatorname{Hom}(M(X), \mathbf{Z}(p)[q])=H^{q}(X, \mathbf{Z}(p))$ (here we take the variance of Voevodsky).

As pointed out at the end of the last subsection, the existence of a "motivic" $t$-structure on $D(k) \otimes \mathbf{Q}$ depends at least on the Beilinson-Soulé Conjecture 2.4.1. In [71, Part III], Hanamura gives a very nice proof of the existence of this $t$-structure for his category, assuming an extension of this conjecture plus Grothendieck's standard conjectures and the Bloch-Beilinson-Murre filtration.

Naturally, in the notation of the previous subsection, one should have $h^{i}(X)=H^{i}\left(M(X)^{\vee}\right)$ and $h_{c}^{i}(X)=H^{i}\left(M^{c}(X)^{\vee}\right)$ for the motivic $t$-structure. The spectral sequence mentioned would just be the corresponding hypercohomology spectral sequence, and its degeneracy for smooth projective $X$ would follow from Grothendieck's standard conjecture B ("Lefschetz type", see [109]) and Deligne's degeneracy criterion [38].

What about a motivic $t$-structure on $D(k)$ itself? In [198, Prop. 4.3.8], Voevodsky shows that there is an obstruction for his category as soon as $c d_{2}(k)>1$. On the other hand, he has an étale version $D M_{\mathrm{gm} \text {,ét }}(k)$ of $D(k)$ $[198, \S 3.3]$. I expect that this category does have an integrally defined motivic $t$-structure: see the next paragraph on Nori's category for more details. Note that the two categories coincide after tensoring morphisms by $\mathbf{Q}$ by $[198, \mathrm{Th}$. $3.3 .2] .^{5}$

Besides the triangulated approach, there have been attempts to construct directly something like $\operatorname{MMot}(k, \mathbf{Q})$. The first idea was to use absolute Hodge cycles à la Deligne (Deligne [36, §1], Jannsen [78, Part I]). This is using cohomology classes rather than algebraic cycles. Another one is to try and construct at least a simpler subcategory of $\operatorname{MMot}(k, \mathbf{Q})$, like the full abelian subcategory generated by Tate motives, using algebraic cycles. This was performed by Bloch-Kriz [23]. On the other hand, Levine proved [115] that conjecture 2.4.1 (for the motivic cohomology of $k$ !) is sufficient to provide a motivic $t$-structure on the thick triangulated category of $D(k) \otimes \mathbf{Q}$ generated by the $\mathbf{Q}(n)$ (this works for any version of $D(k)$, or more generally for any tensor

\footnotetext{
${ }^{5}$ Actually, in loc. cit. a category $D M_{-, \text {ét }}^{\text {eff }}(k)$ analogous to $D M_{-}^{\text {eff }}(k)$ is defined: this is a "big" category. One should probably define $D M_{\mathrm{gm} \text {,ét }}(k)$ as follows: take the full subcategory of $D M_{-}^{\mathrm{eff}}(k)$ generated by the image of $D M_{\mathrm{gm}}^{\mathrm{eff}}(k)$, invert $\mathbf{Z}(1)$ and finally take the pseudo-abelian hull.
} 
triangulated category satisfying suitable axioms). Quite different is Nori's approach, which is described in the next section.

\subsection{Nori's category}

For considerably more details on this subsection, see $\S 3.3$ in Levine's chapter.

Using Betti cohomology, Madhav Nori constructs for any subfield $k$ of $\mathbf{C}$ an abelian, Z-linear category of mixed motives. This exposition is based on notes from a series of lectures he gave in Bombay in 2000: any misunderstanding is of course my own responsibility.

The two fundamental building blocks of Nori's construction are:

Theorem 3.4.1 (Basic lemma). Let $X$ be an affine $k$-variety of dimension $d$, and let $Z$ be a closed subset of $X$ of dimension $<d$. Then there exists a closed subset $Z^{\prime} \supseteq Z$ of dimension $<d$ such that the relative cohomology groups $H^{i}\left(X, Z^{\prime}, \mathbf{Z}\right)$ are 0 for $i \neq d$.

This theorem is stated and proven in [149], but Nori points out that it also follows from earlier work of Beilinson [13, Lemma 3.3], whose proof also works in positive characteristic.

For the next theorem we need the notion of a diagram, also called precategory or quiver: this is the same as a category, except that composition of morphisms is not defined. Any category defines a diagram by forgetting composition, and any functor defines a morphism of diagrams. Let $R$ be a commutative ring, $D$ be a diagram and $T: D \rightarrow R-M o d$ a representation of $D$, that is, a morphism from $D$ to the diagram underlying $R-M o d$. Following Nori, one enriches $T$ as follows: if $D$ is finite, $T$ obviously lifts to a representation $\tilde{T}: D \rightarrow \operatorname{End}_{R}(T)-M o d$, where $\operatorname{End}_{R}(T)$ is the ring of $R$-linear natural transformations from $T$ to itself. In general one may write $D$ as a union of its finite subdiagrams $D^{\prime}$, and define $\mathcal{C}(T)=\varliminf_{\longrightarrow} \operatorname{End}_{R}\left(T_{\mid D^{\prime}}\right)-M o d$. Then there is an obvious forgetful functor $\omega: \mathcal{C}(T) \rightarrow R-M o d$ and $T$ lifts through $\omega$ to

$$
\tilde{T}: D \rightarrow \mathcal{C}(T) .
$$

The following is a universal property of (3.4.1):

Theorem 3.4.2 (Nori's tannakian lemma). Suppose $R$ Noetherian. Let $\mathcal{A}$ be an R-linear abelian category, $\ell: \mathcal{A} \rightarrow R$-Mod an $R$-linear additive faithful exact functor and $S: D \rightarrow \mathcal{A}$ a representation such that $T=\ell S$. Then there exists an $R$-linear exact functor $S^{\prime}: \mathcal{C}(T) \rightarrow \mathcal{A}$, unique up to unique isomorphism, making the following diagram commutative up to natural isomorphism: 


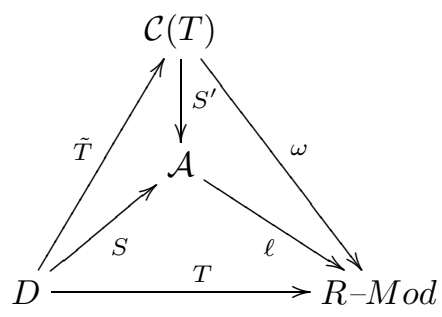

Note that $S^{\prime}$ is automatically faithful since $\omega$ is.

For a proof of this theorem, see Bruguières [29].

(As Srinivas pointed out, the uniqueness statement is not completely correct. For it to be one needs at least $\ell$ to be "totally faithful", a notion introduced by Nori: if an arrow goes to an identity arrow then it is already an identity arrow. This condition is basically sufficient.)

Nori then takes for $D$ the diagram whose objects are triples $(X, Y, i)$ where $X$ is affine of finite type over $k, Y$ is a closed subset and $i \geq 0$, and morphisms are (I) morphisms of triples (same $i$ ) and (II) to any chain $Z \subset Y \subset X$ and integer $i>0$ corresponds a morphism $(X, Y, i) \rightarrow(Y, Z, i-1)$. He takes $T(X, Y, i)=H_{i}(X, Y, \mathbf{Z})$, and also $T^{*}(X, Y, i)=H^{i}(X, Y, \mathbf{Z})$ on the dual diagram $D^{*}$. The corresponding categories $\mathcal{C}(T)$ and $\mathcal{C}\left(T^{*}\right)$ are respectively called EHM(k) (effective homological motives) and ECM $(k)$ (effective cohomological motives).

These categories are independent from the embedding of $k$ into $\mathbf{C}$; $E H M(k)$ is a tensor category and enjoys exact faithful tensor functors to Galois representations, mixed Hodge structures and a category of "periods" (a period is a triple $(M, W, \varphi)$ where $M$ is a $\mathbf{Z}$-module, $W$ a $k$-vector space and $\varphi$ an isomorphism $\left.\mathbf{C} \otimes_{\mathbf{z}} M \rightarrow \mathbf{C} \otimes_{k} W\right)$. There is a tensor triangulated functor

$$
D M_{\mathrm{gm}}^{\mathrm{eff}}(k) \rightarrow D^{b}(E H M(k)) .
$$

One may then define the category $M(k)$ of motives by inverting $\mathbf{Z}(1):=$ $H_{1}\left(\mathbb{G}_{m}\right)$, exactly as for pure motives. Starting from $E C M(k)$ and $H^{1}\left(\mathbb{G}_{m}\right)$ yields the same result. This category is "neutral tannakian" in the sense that it is equivalent to the category of comodules of finite type over a certain pro-Hopf algebra... There is also a weight filtration, all this being integrally defined!

I expect $M(k)$ to be eventually equivalent to the heart of a motivic $t$ structure on $D M_{\mathrm{gm} \text {,ét }}(k)$, compare the end of Subsection 3.3. This is evidenced by the fact that Betti cohomology compares nicely with étale cohomology with finite coefficients. Of course this issue is closely related to the Hodge conjecture.

\section{5 $\mathrm{A}^{\mathbf{1}}$-homotopy and stable homotopy categories}

Before Fabien Morel and Vladimir Voevodsky started constructing it in the early nineties, first independently and then together, nobody had thought of 
developing a "homotopy theory of schemes" just as one develops a homotopy theory of (simplicial) sets. In this subsection we shall give a brief outline of this theory and its stable counterpart, referring the reader to [143], [146] and [201] for details; see also the few words in [89, $\S \S 5$ to 7], [199], the exposition of Joël Riou [158], [144] and the programmatic [145].

\section{Homotopy of schemes}

There are two constructions of the $\mathbf{A}^{1}$-homotopy category of $k$-schemes $\mathcal{H}(k)$, which can be considered as an algebro-geometric generalisation of the classical homotopy category $\mathcal{H}:[143]$ and [146]. It can be shown that they are equivalent. We shall describe the second with its features, as it is the best-known anyway.

We start with the category $(S m / k)_{\text {Nis }}$ of smooth $k$-schemes of finite type endowed with the Nisnevich topology. We first introduce the category $\mathcal{H}_{s}(k)$. A map $f: \mathcal{F} \rightarrow \mathcal{G}$ of Nisnevich sheaves of simplicial sets on $(S m / k)_{\text {Nis }}$ is a simplicial weak equivalence if $f_{x}: \mathcal{F}_{x} \rightarrow \mathcal{G}_{x}$ is a weak equivalence of simplicial sets for any point $x$ of the site; $\mathcal{H}_{s}(k)$ is the localisation of $\Delta^{\mathrm{op}} \operatorname{Shv}\left((S m / k)_{\mathrm{Nis}}\right)$ with respect to simplicial weak equivalences. Next, a simplicial sheaf $\mathcal{F}$ is $\mathbf{A}^{1}$. local if for any other simplicial sheaf $\mathcal{G}$, the map

$$
\operatorname{Hom}_{\mathcal{H}_{s}(k)}(\mathcal{G}, \mathcal{F}) \rightarrow \operatorname{Hom}_{\mathcal{H}_{s}(k)}\left(\mathcal{G} \times \mathbf{A}^{1}, \mathcal{F}\right)
$$

induced by the projection $\mathbf{A}^{1} \rightarrow$ Spec $k$ is a bijection, and a morphism $f$ : $\mathcal{G} \rightarrow \mathcal{H}$ is an $\mathbf{A}^{1}$-weak equivalence if for any $\mathbf{A}^{1}$-local $\mathcal{F}$ the corresponding map

$$
f^{*}: \operatorname{Hom}_{\mathcal{H}_{s}(k)}(\mathcal{H}, \mathcal{F}) \rightarrow \operatorname{Hom}_{\mathcal{H}_{s}(k)}(\mathcal{G}, \mathcal{F})
$$

is a bijection. Then $\mathcal{H}\left(k, \mathbf{A}^{1}\right)=\mathcal{H}(k)$ is the localisation of $\Delta^{\mathrm{op}} \operatorname{Shv}\left((S m / k)_{\text {Nis }}\right)$ with respect to $\mathbf{A}^{1}$-weak equivalences.

Morel and Voevodsky provide $\Delta^{\mathrm{op}} \operatorname{Shv}\left((S m / k)_{\mathrm{Nis}}\right)$ with a closed model structure of which $\mathcal{H}(k)$ is the homotopy category. By construction, any $k$ scheme of finite type can be viewed in $\mathcal{H}(k)$. For smooth schemes, this transforms elementary Nisnevich covers (for example open covers by two Zariski open sets) into cocartesian squares, the affine line is contractible, one has a homotopy cocartesian blow-up square (up to a suspension) and a homotopy purity theorem for a smooth pair, expressible in terms of the Thom space of the normal bundle.

Moreover, most of the important cohomology theories are representable in $\mathcal{H}(k)$ : this is the case in particular for algebraic $K$-theory and for motivic cohomology.

When $k \subseteq \mathbf{C}$, there is a realisation functor $\mathcal{H}(k) \rightarrow \mathcal{H}$; when $k \subseteq \mathbf{R}$ there is another one, quite different from the first.

In contrast to the classical case of $\mathcal{H}$, there are two circles in $\mathcal{H}(k)$ : the simplicial circle $S_{s}^{1}$ and the $\mathbf{A}^{1}$-circle $S_{t}^{1}$. They account for the fact that motivic cohomology is a bigraded theory; the fact that algebraic $K$-theory is singleindexed can be interpreted as an algebraic analogue to Bott periodicity. 


\section{Stable homotopy of schemes}

The construction of this category is outlined in [201]; see also [89, $5.4 \mathrm{ff}]$, [144], [145] and [158]. Briefly, one considers $T$-spectra, where $T=S_{s}^{1} \wedge S_{t}^{1}$ and one constructs $\mathcal{S H}(k)$ "as in topology", except that "as in" hides not inconsiderable technical difficulties. This is a tensor triangulated category, just as the classical stable homotopy category $\mathcal{S H}$. There exists an infinite version of $D M_{\mathrm{gm}}(k)$, denoted by $D M(k)$ ([179], [210], [145, §5.2]), to which $\mathcal{S H}(k)$ bears the same relationship as $\mathcal{S H}$ bears to the derived category of abelian groups: there is a "motive" functor

$$
M: \mathcal{S H}(k) \rightarrow D M(k)
$$

which extends the functor $M: S m / k \rightarrow D M_{\mathrm{gm}}^{\mathrm{eff}}(k)$ (an analogue to the "chain complex" functor in topology), and which has as a right adjoint an "EilenbergMacLane" functor

$$
H: D M(k) \rightarrow \mathcal{S H}(k) .
$$

Moreover, if one tensors morphisms by $\mathbf{Q}$, then $H$ is a right inverse to $M$; if -1 is a sum of squares in $k, H$ is even an inverse to $M$.

An important theorem is that motivic cohomology is representable by a $\Omega_{T}$-spectrum [201, Th. 6.2]: this rests on Voevodsky's cancellation theorem [202]. Similarly, $K$-theory is representable by a $\Omega_{T}$-spectrum $K G L$. This has allowed Hopkins and Morel to construct an Atiyah-Hirzebruch spectral sequence having the same form as (2.2.3), by using a tower on $K G L$ rather than the "skeleta" approach leading to (2.2.3) [75]. Unlike the topological case, it is not clear that the two spectral sequences coincide ${ }^{6}$.

To prove Theorem 4.2.8 below, Voevodsky makes an essential use of the categories $\mathcal{H}(k)$ and $\mathcal{S H}(k)$, and in particular of motivic Steenrod operations.

\section{Comparisons}

\section{1 Étale topology}

Instead of computing higher Chow groups for the Zariski topology, we may use étale topology. For $X$ smooth and $n \geq 0$, we thus get groups

$$
H_{\text {êt }}^{i}(X, \mathbf{Z}(n)):=\mathbb{H}_{\text {ét }}^{i-2 n}\left(X, \alpha^{*} z^{n}(X, *)\right)
$$

${ }^{6}$ In the topological case, one can easily prove that the two ways to produce the Atiyah-Hirzebruch spectral sequence for a generalised cohomology theory (cell filtration on the space or Postnikov tower on the spectrum) yield the same spectral sequence by reducing to the case where the space is a sphere and where the spectrum is an Eilenberg-Mac Lane spectrum, because the cohomology of spheres is so basic. In the scheme-homotopy theoretic case, even if one had the suitable generality of construction of the two spectral sequences, one would have to find the right analogues: for example, motivic cohomology of fields is quite complicated. 
where $\alpha$ is the projection $(S m / k)_{\text {ét }} \rightarrow(S m / k)_{\text {Zar }}$. There are canonical maps $H^{i}(X, \mathbf{Z}(n)) \rightarrow H_{\text {ét }}^{i}(X, \mathbf{Z}(n))$. Similarly, replacing the complexes $z_{n}(X, *)$ by $z_{n}(X, *) \otimes \mathbf{Z} / m$, we may define motivic cohomology with finite coefficients, both in the Zariski and the étale topology:

$$
H^{i}(X, \mathbf{Z} / m(n)) \rightarrow H_{\text {êt }}^{i}(X, \mathbf{Z} / m(n)) .
$$

Theorem 4.1.1 (Geisser-Levine). Let $X$ be smooth over $k$.

a) [62, Th. 1.5] If $m$ is invertible in $k$, there is a quasi-isomorphism $\underline{z}_{n}(-, *)_{\text {ét }} \otimes \mathbf{Z} / m \simeq \mu_{m}^{\otimes n}$.

b) [61] If $m$ is a power of $p=\operatorname{char} k>0$, say $m=p^{s}$, there is a quasiisomorphism $\underline{z}_{n}(-, *)$ ét $\otimes \mathbf{Z} / m \simeq \nu_{s}(n)[-n]$, where $\nu_{s}(n)$ is the $n$-th logarithmic Hodge-Witt sheaf of level s. ${ }^{7}$

\subsection{Zariski topology}

Keep the above notation. We first have an easy comparison with rational coefficients (e.g. [93, Prop. 1.18]):

Theorem 4.2.1. For $X$ smooth over $k, H^{i}(X, \mathbf{Q}(n)) \stackrel{\sim}{\longrightarrow} H_{\text {ét }}^{i}(X, \mathbf{Q}(n))$ for all $i, n$.

Theorem 4.2.2 (Geisser-Levine [61, Th. 8.4]). Let $X$ be smooth over $k$. If $m=p^{s}, p=\operatorname{char} k>0, H_{\mathrm{Zar}}^{i}(X, \mathbf{Z} / m(n)) \simeq H_{\mathrm{Zar}}^{i-n}\left(X, \alpha_{*} \nu_{s}(n)\right)$.

See $§ 3.2$ in Geisser's chapter for more details on this theorem.

For $m$ invertible in $k$, the situation is more conjectural. Let $l$ be prime and invertible in $k$. Recall

Bloch-Kato conjecture 4.2.3. For any finitely generated extension $K / k$ and any $n \geq 0$, the norm residue homomorphism

$$
K_{n}^{M}(K) / l \rightarrow H_{\text {ét }}^{n}\left(K, \mu_{l}^{\otimes n}\right)
$$

is bijective.

The references for this conjecture are [100, Conj. 1] and [19, Intr. and Lect. 5]. For $l=2$, it is due to Milnor [140, p. 540].

Beilinson-Lichtenbaum conjecture 4.2.4. For any smooth $X$ over $k$, the quasi-isomorphism of Theorem 4.1.1 a) induces a quasi-isomorphism $\underline{z}_{n}(-, *) \otimes \mathbf{Z} / m \simeq \tau_{\leq n} R \alpha_{*} \mu_{m}^{\otimes n}$

The references for this conjecture are $[12,5.10 \mathrm{D}$ (vi)] and $[124, \S 5]$. In view of Theorems 4.2.1 and 4.2.2, it may be reformulated as follows:

7 This statement does not appear explicitly in [61]; however it can be deduced from Theorem 4.2 .2 by étale localisation! 
Lemma 4.2.5. Conjecture 4.2.4 is equivalent to the following statement: for any smooth $k$-variety $X$, the natural morphism

$$
\mathbf{Z}(X, n) \rightarrow \tau_{\leq n+1} R \alpha_{*} \alpha^{*} \mathbf{Z}(X, n)
$$

is an isomorphism in $D^{-}\left(X_{\text {Zar }}\right)$, where $\mathbf{Z}(X, n)$ is the class of the Zariski sheafification of the shifted Bloch cycle complex $z^{*}(X, n)[-2 n]$ and where $\alpha$ is the projection of the small étale site of $X$ onto its small Zariski site.

(The vanishing of $R^{n+1} \alpha_{*} \alpha^{*} \mathbf{Z}(X, n)$ is called Hilbert 90 in weight $n$.)

The following theorem is due to Suslin-Voevodsky [186] in characteristic 0 and to Geisser-Levine [62] in general.

Theorem 4.2.6. Conjectures 4.2.3 and 4.2.4 are equivalent.

The arguments of [61] for the proof of Theorem 4.2.2 and of [186] and [62] for the proof of Theorem 4.2.6 can be abstracted [93] and give a uniqueness theorem for motivic cohomology. Let us explain this theorem. Define (in this explanation) a cohomology theory as a sequence of complexes of sheaves $(B(n))_{n \in \mathbf{Z}}$ over the category of smooth $k$-schemes endowed with the Zariski topology, enjoying two natural properties: homotopy invariance and Gysin exact sequences (purity). There is an obvious notion of morphisms of cohomology theories. For example, $(\mathbf{Z}(n))_{n \in \mathbf{Z}}$ defines a cohomology theory as soon as we modify Bloch's cycle complexes suitably so as to make them strictly contravariant for morphisms between smooth varieties [93, Th. 1.17] ${ }^{8}$. Suppose now that $C$ is a bounded above complex of abelian groups (in applications to Theorems 4.2.2 and 4.2.6, $C$ would be $\mathbf{Z} / m$ for some $m$ ) and that we are given a morphism of cohomology theories

$$
\mathbf{Z}(n) \stackrel{L}{\otimes} C \rightarrow B(n) .
$$

Under what conditions is (4.2.1) an isomorphism? Two obvious necessary conditions are the following:

1. $B(n)$ is bounded above for all $n$.

2. (4.2.1) is a quasi-isomorphism for $n \leq 0$; in particular, $B(n)=0$ for $n<0$.

The third condition is very technical to state: it is called malleability and is enjoyed by $\mathbf{Z}(n)$ for $n>0$ by a nontrivial theorem of Geisser and Levine ([61, Cor. 4.4], [93, Th. 2.28]). For $n=1$ it is closely related to the fact that, if $A$ is a semi-local ring, then for any ideal $I$ in $A$ the homomorphism $A^{*} \rightarrow(A / I)^{*}$ is surjective. It is stable under tensoring with $C$, so if (4.2.1) is an isomorphism then

\footnotetext{
${ }^{8}$ One should be careful that the construction given in [93] is incomplete. The problem is that the claimed equality in loc. cit., (1.4) is only an inclusion in general. As a consequence, the object defined in the proof of Theorem 1.17 is not a functor, but only a lax functor. This lax functor can be rectified e.g. by the methods of [122] (see also Vogt [206]). I am grateful to Marc Levine for pointing out this gap, and the way to fill it.
} 
3. $B(n)$ is malleable for all $n>0$.

Conversely:

Theorem 4.2.7 ([93, Prop. 2.30]). If Conditions 1, 2 and 3 are satisfied, then (4.2.1) is an isomorphism of cohomology theories.

Finally, we have the celebrated theorem of Voevodsky:

Theorem 4.2.8 (Voevodsky [203]). Conjecture 4.2.3 is true for $l=2$.

The reader can have a look at [50], [89], [142] and [183] for some insights in the proof.

For an odd prime $l$, Conjecture 4.2.3 is "proven" in the following sense: 1) A preprint of Voevodsky [205] gives a proof modulo two lemmas on mod $l$ Steenrod operations (see loc. cit. , Lemmas 2.2 and 2.3) and two results of Rost (see loc. cit., Th. 6.3). 2) Voevodsky plans to write up a proof of the Steenrod operation lemmas, but no such proof is available at the moment. 3) The two results of Rost have been announced by him in his address to the Beijing International Congress of Mathematicians in 2002 with proofs of special cases [161], but no complete proof is available at the moment.

Corollary 4.2.9. For $n$ a power of 2 (or more generally for all $n$ with the caveat just above), the $E_{2}$-terms of the spectral sequence (2.2.4) have the form

$$
E_{2}^{p, q}=H_{\text {Zar }}^{p}\left(X, \tau_{\leq-q / 2} R \alpha_{*} \mu_{n}^{\otimes(-q / 2)}\right) .
$$

In particular, if $X$ is semi-local, then

$$
E_{2}^{p, q}= \begin{cases}H_{\mathrm{ét}}^{p}\left(X, \mu_{n}^{\otimes(-q / 2)}\right) & \text { if } p \leq-q / 2 \\ 0 & \text { if } p>-q / 2 .\end{cases}
$$

Corollary 4.2.10 (Compare [120, proof of Cor 13.3]). Let $X$ be a connected regular scheme essentially of finite type over a field or a Dedekind scheme $S$ Let $\delta$ be the étale cohomological 2-dimension of the function field of $X$ and $d=\operatorname{dim} X$. Then, for any $n \geq 0, H^{i}\left(X, \mathbf{Z} / 2^{s}(n)\right)=0$ for $i>\delta+d$.

Proof. We may assume $\delta<\infty$. Consider the hypercohomology spectral sequence

$$
E_{2}^{p, q}=H_{\text {Zar }}^{p}\left(X, \mathcal{H}^{q}\left(\mathbf{Z} / 2^{s}(n)\right)\right) \Rightarrow H^{p+q}\left(X, \mathbf{Z} / 2^{s}(n)\right) .
$$

It is sufficient to show that $E_{2}^{p, q}=0$ for $p+q>\delta+d$. We distinguish two cases:

- $q>n$. Then it follows from the definition of Bloch's higher Chow groups plus Gersten's conjecture [32], which implies that the stalks of the sheaf $\mathcal{H}^{q}\left(\mathbf{Z} / 2^{s}(n)\right)$ inject into its stalk at the generic point.

- $q \leq n$. By Theorems 4.2.6 and 4.2.8, $\mathcal{H}^{q}\left(\mathbf{Z} / 2^{s}(n)\right) \stackrel{\sim}{\longrightarrow} \mathcal{H}_{\text {êt }}^{q}\left(\mathbf{Z} / 2^{s}(n)\right)$. But the right sheaf is 0 for $q>\delta$ by the argument in [91, Proof of Cor. 4.2]. 


\subsection{Back to the Beilinson-Soulé conjecture}

Lemma 4.3.1. Under Conjecture 4.2.4, Conjecture 2.4.1 is equivalent to Conjecture 2.4.1 tensored by $\mathbf{Q}$.

Indeed, Conjecture 4.2.4 implies that $H^{i}(X, \mathbf{Z} / m(n))=H_{\text {ét }}^{i}\left(X, \mu_{m}^{\otimes n}\right)=0$ for $i<0$ and any $m>0$. This in turn implies that $H^{i}(X, \mathbf{Z}(n)) \rightarrow$ $H^{i}(X, \mathbf{Q}(n))$ is an isomorphism for any $i<0$ and is injective for $i=0$.

\subsection{Borel-Moore étale motivic homology}

For the sequel we shall need a Borel-Moore homology theory that has the same relationship to étale motivic cohomology as ordinary Borel-Moore motivic homology has to ordinary motivic cohomology. Ideally, we would like to associate to any $k$-scheme of finite type $X$ a collection of abelian groups $H_{i}^{B M, \text { ét }}(X, \mathbf{Z}(n))$ enjoying the following two properties:

1. Poincaré duality: if $X$ is a closed subscheme of a smooth $k$-scheme $M$ of dimension $d$, we have isomorphisms

$$
H_{i}^{B M, \text { ét }}(X, \mathbf{Z}(n)) \simeq H_{X \text {,ét }}^{2 d-i}(M, \mathbf{Z}(d-n))
$$

where the right hand side is étale hypercohomology of $M$ with supports in $X$.

2. Localisation: if $Z$ is a closed subset of $X$ and $U=X-Z$, we have long exact sequences

$$
\begin{aligned}
& \cdots \rightarrow H_{i}^{B M, \text { ét }}(Z, \mathbf{Z}(n)) \rightarrow H_{i}^{B M, \text { ét }}(X, \mathbf{Z}(n)) \\
& \rightarrow H_{i}^{B M \text {,ét }}(U, \mathbf{Z}(n)) \rightarrow H_{i-1}^{B M \text {,ét }}(Z, \mathbf{Z}(n)) \rightarrow \ldots
\end{aligned}
$$

The problem is that 1 ) does not make sense a priori because if $q<0$ and $M$ is smooth the groups $H_{\text {êt }}^{j}(M, \mathbf{Z}(q))$ have not been defined. For $n \in \mathbf{Z}$, let

$$
\begin{aligned}
(\mathbf{Q} / \mathbf{Z})^{\prime}(n) & :=\underset{(m, \mathrm{char} k)=1}{\lim _{m}} \mu_{m}^{\otimes n} \\
\mathbf{Q}_{p} / \mathbf{Z}_{p}(n) & :=\left\{\begin{array}{ll}
\underset{\lim }{\longrightarrow} \nu_{s}(n)[-n] & \text { for } n \geq 0 \\
0 & \text { for } n<0
\end{array} \text { if } \operatorname{char} k=p>0\right. \\
\mathbf{Q} / \mathbf{Z}(n) & :=(\mathbf{Q} / \mathbf{Z})^{\prime}(n) \oplus \mathbf{Q}_{p} / \mathbf{Z}_{p}(n) .
\end{aligned}
$$

Theorems 4.1.1 and 4.2.1 imply that for $X$ smooth and $n \geq 0$ we have long exact sequences

$$
\begin{aligned}
\cdots \rightarrow H_{\text {êt }}^{i-1}(X, \mathbf{Q} / \mathbf{Z}(n)) & \rightarrow H_{\text {ét }}^{i}(X, \mathbf{Z}(n)) \\
& \rightarrow H^{i}(X, \mathbf{Q}(n)) \rightarrow H_{\text {êt }}^{i}(X, \mathbf{Q} / \mathbf{Z}(n)) \rightarrow \ldots
\end{aligned}
$$


where so that we might try and define

$$
H_{\text {ét }}^{i}(X, \mathbf{Z}(n)):=H_{\text {ét }}^{i-1}(X, \mathbf{Q} / \mathbf{Z}(n)) \quad \text { for } X \text { smooth and } n<0 .
$$

This is vindicated by the projective bundle formula

$$
H_{\mathrm{et}}^{i}\left(\mathbf{P}_{X}^{n}, \mathbf{Z}(q)\right) \simeq \bigoplus_{j=0}^{n} H_{\mathrm{ét}}^{i-2 j}(X, \mathbf{Z}(q-j))
$$

which may be proven as [86, Th. 5.1].

Suppose that in 1) $X$ is smooth of dimension $d$. Then we may take $M=X$ and we get isomorphisms $H_{i}^{B M \text {,ét }}(X, \mathbf{Z}(n)) \simeq H_{\text {ét }}^{2 d-i}(X, \mathbf{Z}(d-n))$. Suppose now that in 2) $Z$ is also smooth, of codimension $c$. Then, after reindexing, the localisation exact sequences translate into long exact "Gysin" sequences

$$
\begin{aligned}
& \cdots \rightarrow H_{\text {ét }}^{j-2 c}(Z, \mathbf{Z}(m-c)) \rightarrow H_{\text {êt }}^{j}(X, \mathbf{Z}(m)) \\
& \quad \rightarrow H_{\text {ét }}^{j}(U, \mathbf{Z}(m)) \rightarrow H_{\text {ét }}^{j-2 c+1}(Z, \mathbf{Z}(m-c)) \rightarrow \ldots
\end{aligned}
$$

However, with Definition (4.4.3) the exact sequences (4.4.4) are wrong in characteristic $p>0$ : take for example $k$ algebraically closed, $X=\mathbf{P}^{1}, U=$ $\mathbf{A}^{1}, m=0$ and $j=2$. The exactness of (4.4.4) would imply that the homomorphism $H_{\text {ét }}^{1}\left(\mathbf{P}^{1}, \mathbf{Q} / \mathbf{Z}\right) \rightarrow H_{\text {ét }}^{1}\left(\mathbf{A}^{1}, \mathbf{Q} / \mathbf{Z}\right)$ is surjective, which is false because $H_{\text {ét }}^{1}\left(\mathbf{P}^{1}, \mathbf{Q}_{p} / \mathbf{Z}_{p}\right)=0$ while $H_{\text {ét }}^{1}\left(\mathbf{A}^{1}, \mathbf{Q}_{p} / \mathbf{Z}_{p}\right)$ is huge. Therefore the most we can hope for is to have exact sequences (4.4.4) after inverting the exponential characteristic $p$.

This turns out to be true: (4.4.4) $\otimes \mathbf{Z}[1 / p]$ are obtained by gluing together the purity theorem for motivic cohomology (2.4.1) and the purity theorem for étale cohomology with finite coefficients, as in [86, Th. 4.2].

It also turns out that a Borel-Moore homology theory

$$
X \mapsto\left(H_{i}^{B M, \text { ét }}(X, \mathbf{Z}[1 / p](n))\right)_{(i, n) \in \mathbf{Z} \times \mathbf{Z}}
$$

having properties 1) and 2) after inverting $p$ does exist. These groups sit in long exact sequences analogous to (4.4.2)

$$
\begin{aligned}
\cdots \rightarrow H_{i+1}^{B M, \text { ét }} & \left(X,(\mathbf{Q} / \mathbf{Z})^{\prime}(n)\right) \rightarrow H_{i}^{B M, \text { ét }}(X, \mathbf{Z}[1 / p](n)) \\
& \rightarrow H_{i}^{B M}(X, \mathbf{Q}(n)) \rightarrow H_{i}^{B M, \text { ét }}\left(X,(\mathbf{Q} / \mathbf{Z})^{\prime}(n)\right) \rightarrow \ldots
\end{aligned}
$$

where $H_{i+1}^{B M, \text { ét }}\left(X,(\mathbf{Q} / \mathbf{Z})^{\prime}(n)\right)=H^{-i-1}\left(X, f_{X}^{!}(\mathbf{Q} / \mathbf{Z})^{\prime}(-n)\right)$ is étale BorelMoore homology, with $f_{X}^{!}$the extraordinary inverse image of [SGA 4, Exposé XVIII] associated to $f_{X}: X \rightarrow$ Spec $k$. Supposing that they are constructed, they are characterised either by (4.4.1) (with $p$ inverted) or by (4.4.6).

I know two techniques to construct the theory (4.4.5). The first is to proceed "naïvely" as in [62] and construct a homotopy version of the homological 
cycle class map of [SGA $41 / 2$, Cycle, $\S 2.3]$ : this had been done in [92, §1.3]. This yields functorial zig-zags of morphisms

$$
\alpha^{*} z_{n}(X, *) \stackrel{\sim}{\longleftarrow} f_{X}^{!} \mathbf{Z} / m(-n)[-2 n]
$$

for $(m, \operatorname{char} k)=1$, which are compatible when $m$ varies; in the limit one gets zig-zags

$$
\alpha^{*} z_{n}(X, *) \otimes \mathbf{Q} \stackrel{\sim}{\longleftarrow} f_{X}^{!}(\mathbf{Q} / \mathbf{Z})^{\prime}(-n)[-2 n]
$$

and one defines (4.4.5) as the homology groups of the homotopy fibre. The other method is much more expensive but also more enlightening. First, one proves that the cohomology theory on smooth schemes

$$
X \mapsto\left(H_{\text {ét }}^{i}(X, \mathbf{Z}[1 / p](n))\right)_{(i, n) \in \mathbf{Z} \times \mathbf{Z}}
$$

is representable by a $T$-spectrum $H_{\mathbf{Z}[1 / p]}^{\text {et }}$ in $\mathcal{S H}(k)$ : for this one may glue the $T$-spectrum $H_{\mathbf{Q}}$ with the $T$-spectrum $H_{(\mathbf{Q} / \mathbf{z})^{\prime}}^{\text {et }}$ representing étale cohomology with $(\mathbf{Q} / \mathbf{Z})^{\prime}$ coefficients ( $c f$. [158, Cor. 8.53]) in the spirit above, except that it is much easier here. (As Joël Riou pointed out, it is even easier to apply the Dold-Kan construction to truncations of Godement resolutions representing $R \alpha_{*} \alpha^{*} \mathbf{Z}[1 / p](n)$ : the projective bundle formula and homotopy invariance imply that they yield an $\Omega_{T}$-spectrum.) Then, according to Voevodsky's formalism of cross functors $(c f$. $[204,9])$, given a $k$-scheme of finite type $X$ with structural morphism $f: X \rightarrow \operatorname{Spec} k$, we have an "extraordinary direct image" functor

$$
f_{!}: \mathcal{S H}(X) \rightarrow \mathcal{S H}(k) .
$$

We set $B M(X)=f_{!} S^{0}$ : this is the Borel-Moore object associated to $X$. For any $T$-spectrum $E \in \mathcal{S H}(k)$, we may then define

$$
E_{p, q}^{B M}(X):=\left[\Sigma^{-p,-q} B M(X), E\right]
$$

(I am indebted to Riou for discussions about this.) Applying this to $E=$ $H_{\mathbf{Z}[1 / p]}^{e ́ t}$, we get the desired theory.

Note that, for a singular scheme $X$, one may also consider the groups

$$
H_{\text {ét }}^{2 n-i}\left(X, \alpha^{*} z_{n}(X, *)[1 / p]\right)
$$

obtained by sheafifying the Bloch cycle complexes for the étale topology. These groups map to $H_{i}^{B M, \text { et }}(X, \mathbf{Z}[1 / p](n))$, but these maps are not isomorphisms in general as one can see easily because of (4.4.1). So the isomorphism of [92, (1.6)] is wrong. (I am indebted to Geisser for pointing out this issue.) However they become isomorphisms after tensoring with $\mathbf{Q}$, and these groups then reduce to Bloch's higher Chow groups tensored with $\mathbf{Q}$.

Finally, one can repeat the story above after tensoring the étalified Bloch cycle complexes (for smooth schemes) by a fixed complex of étale sheaves $C$ on the small étale site of Spec $k$ : this will be used in the sequel. 


\section{Applications: local structure of algebraic $K$-groups and finiteness theorems}

Definition 5.0.1. Let $X$ be a $\operatorname{Spec} \mathbf{Z}[1 / 2]$-scheme: it is non-exceptional if for any connected component $X_{\alpha}$, the image of the cyclotomic character $\kappa_{2}$ : $\pi_{1}\left(X_{\alpha}\right) \rightarrow \mathbf{Z}_{2}^{*}$ does not contain -1 .

The first result says that, locally for the Zariski topology, algebraic $K$ theory with $\mathbf{Z} / 2^{\nu}$ coefficients is canonically a direct sum of étale cohomology groups, at least in the nonexceptional case:

Theorem 5.0.2 ([91, Th. 1]). Let $A$ be a semi-local non-exceptional $\mathbf{Z}[1 / 2]$ algebra.

a) There are canonical isomorphisms $(n \geq 0, \nu \geq 2)$

$$
\coprod_{0 \leq i \leq n} H_{\text {ét }}^{2 i-n}\left(A, \mu_{2^{\nu}}^{\otimes i}\right) \stackrel{\sim}{\longrightarrow} K_{n}\left(A, \mathbf{Z} / 2^{\nu}\right) .
$$

b) If $A$ is essentially smooth over field or a discrete valuation ring, the spectral sequence (2.2.2) with $\mathbf{Z} / 2^{\nu}$ coefficients canonically degenerates.

c) If $A$ is a field and $\mu_{2^{\nu}} \subset A$, the natural map

$$
K_{*}^{M}(A) \otimes \mathbf{Z} / 2^{\nu}[t] \rightarrow K_{*}\left(A, \mathbf{Z} / 2^{\nu}\right)
$$

given by mapping t to a "Bott element" is an isomorphism.

Note that the reason why Thomason disbelieved the Bloch-Kato conjecture was precisely that it would imply the vanishing of all differentials in the Atiyah-Hirzebruch spectral sequence for étale $K$-theory [194, p. 409]: similar results had been observed by Dwyer-Friedlander [45]. See $\S 3$ in Weibel's chapter for details on the construction of the isomorphism a) in some special cases.

For $X$ a scheme, define $d_{2}(X):=\sup \left\{c d_{2}(\eta)\right\}$, where $\eta$ runs through the generic points of $X$.

Theorem 5.0.3 (ibid. , Th. 2). Let $X$ be a finite-dimensional Noetherian non-exceptional $\mathbf{Z}[1 / 2]$-scheme.

a) The natural map

$$
K_{n}^{T T}\left(X, \mathbf{Z} / 2^{\nu}\right) \rightarrow K_{n}^{T T}\left(X, \mathbf{Z} / 2^{\nu}\right)\left[\beta^{-1}\right]
$$

is injective for $n \geq \sup \left(d_{2}(X)-2,1\right)$ and bijective for $n \geq \sup \left(d_{2}(X)-1,1\right)$. The 1 in the sup is not necessary if $X$ is regular. (Recall that $K^{T T}$ denotes Thomason-Trobaugh K-theory.)

b) The natural map

$$
K_{n}^{\prime}\left(X, \mathbf{Z} / 2^{\nu}\right) \rightarrow K_{n}^{\prime}\left(X, \mathbf{Z} / 2^{\nu}\right)\left[\beta^{-1}\right]
$$

is injective for $n \geq d_{2}(X)-2$ and bijective for $n \geq d_{2}(X)-1$.

c) If $c d_{2}(X)<+\infty$, there are isomorphisms 


$$
K_{n}^{T T}\left(X, \mathbf{Z} / 2^{\nu}\right)\left[\beta^{-1}\right] \stackrel{\sim}{\longrightarrow} K_{n}^{\text {ét }}\left(X, \mathbf{Z} / 2^{\nu}\right)
$$

for all $n \in \mathbf{Z}$.

Remark 5.0.4. For $X$ regular over a field or a discrete valuation ring, one can directly use the spectral sequences (2.2.2) and (2.2.3) with finite coefficients, $c f$. Levine [120]; but this approach does not work for singular $X$ and $K^{T T}$.

Corollary 5.0.5 (ibid. , Cor. 1). Let $S$ be $\mathbf{Z}[1 / 2]$-scheme and $X$ a nonexceptional separated $S$-scheme of finite type. Assume that $S$ is

(i) Spec $R[1 / 2]$, where $R$ the ring of integers of a non-exceptional number field, or

(ii) $\mathrm{Spec}_{\mathrm{F}}, p>2$, or

(iii) Spec $k, k$ separably closed field of characteristic $\neq 2$, or

(iv) Spec $k, k$ a higher local field in the sense of Kato.

Then $K_{n}^{T T}\left(X, \mathbf{Z} / 2^{\nu}\right)$ and $K_{n}^{\prime}\left(X, \mathbf{Z} / 2^{\nu}\right)$ are finite for $n \geq \operatorname{dim}(X / S)+d_{2}(S)-$ 2 .

Remark 5.0.6. For $X$ regular, the map $H^{i}\left(X, \mathbf{Z} / 2^{\nu}(n)\right) \rightarrow H_{\text {ét }}^{i}\left(X, \mu_{2^{\nu}}^{\otimes n}\right)$ is injective for $i \leq n+1$ (even an isomorphism for $i \leq n$ ) by Conjecture 4.2.4 and Theorem 4.2.8, hence $H^{i}\left(X, \mathbf{Z} / 2^{\nu}(n)\right)$ is finite by Deligne's finiteness theorem for étale cohomology [SGA $41 / 2$, th. finitude] plus arithmetic finiteness theorems. This remark yields Corollary 5.0 .5 by applying Corollaries 4.2 .9 and 4.2.10. The general case needs the methods of [91].

Corollary 5.0.7 (ibid. , Cor. 2). Let $X$ be a variety of dimension d over $k=\mathbf{F}_{p}$ (resp. $\left.\mathbf{Q}_{p}\right), p>2$. Then $K_{n}^{T T}(X)\{2\}$ is finite and $\frac{K_{n+1}^{T T}(X)}{K_{n+1}^{T T}(X)\{2\}}$ is uniquely 2-divisible for $n \geq d$ (resp. $d+1$ ). The same statements hold with $K_{*}^{\prime}(X)$.

Theorem 5.0.8 (ibid. , Th. 3). Let $d \geq 0$ and $n \geq 3$. There exists an effectively computable integer $N=N(d, n)>0$ such that, for any Noetherian $\mathbf{Z}[1 / 2]$-scheme $X$ separated of Krull dimension $\leq d$ and all $\nu \geq 2$, the kernel and cokernel of the map

$$
K_{n}^{T T}\left(X, \mathbf{Z} / 2^{\nu}\right) \stackrel{\left(i c_{i, 2 i-n}\right)}{\longrightarrow} \prod_{i \geq 1} H_{\mathrm{Zar}}^{2 i-n}\left(X, B / 2^{\nu}(i)\right)
$$

(given by Chern classes) are killed by $N$. If $X$ is smooth over a field or a discrete valuation ring, this holds also for $n=2$.

Let us come back to Conjecture 2.3.1 in the light of this theorem. If $X$ is of finite type over a field $k$ of characteristic 0 , the construction of the Haesemeyer spectral sequence (2.3.2) yields a version with coefficients $\mathbf{Z} / 2^{\nu}$. The abutment is $K_{*}^{T T}\left(X, \mathbf{Z} / 2^{\nu}\right)$ by homotopy invariance of the latter theory. The $E_{2}$-terms are of the form $H_{c d h}^{p}\left(X, \theta^{*} B / 2^{\nu}(-q / 2)\right)$, where $\theta$ is the projection of the cdh site of $k$ onto its big Zariski site. This looks closely like 
the spectral sequence in Conjecture 2.3.1. Is there any reason why the maps $H_{\mathrm{Zar}}^{p}\left(X, B / 2^{\nu}(-q / 2)\right) \rightarrow H_{c d h}^{p}\left(X, \theta^{*} B / 2^{\nu}(-q / 2)\right)$ should be isomorphisms? A moment of reflection suggests that there might be a base change theorem between étale and cdh topology (involving Geisser's éh topology [60]) which should be closely related to Gabber's affine analogue of proper base change $[55]$.

If $X=\operatorname{Spec} O_{S}$ where $O_{S}$ is a localised ring of integers in a global field (with $\left.1 / 2 \in O_{S}\right)$ and $O_{S}$ is not formally real, then $\operatorname{cd}_{2}\left(O_{S}\right)=2$ and the spectral sequence (2.2.4) degenerates for dimension reasons. Hence Corollary 4.2 .9 directly yields isomorphisms

$$
\begin{aligned}
& K_{2 i-1}\left(O_{S}, \mathbf{Z} / 2^{\nu}\right) \simeq H_{\text {ét }}^{1}\left(O_{S}, \mu_{2^{\nu}}^{\otimes i}\right) \\
& K_{2 i-2}\left(O_{S}, \mathbf{Z} / 2^{\nu}\right) \simeq H_{\text {ét }}^{2}\left(O_{S}, \mu_{2^{\nu}}^{\otimes i}\right) \quad(i \geq 2) .
\end{aligned}
$$

This is a finite coefficients version of the original Quillen conjecture $(c f$. Conjecture 2.1.1)

$$
\begin{aligned}
& K_{2 i-1}\left(O_{S}\right) \otimes \mathbf{Z}_{l} \simeq H_{\text {ét }}^{1}\left(O_{S}, \mathbf{Z}_{l}(i)\right) \\
& K_{2 i-2}\left(O_{S}\right) \otimes \mathbf{Z}_{l} \simeq H_{\text {ét }}^{2}\left(O_{S}, \mathbf{Z}_{l}(i)\right) \quad(i \geq 2)
\end{aligned}
$$

The latter readily follows from the finite version by passing to the inverse limit, because of the finiteness of the étale cohomology groups and Quillen's finite generation theorem (see below).

When $O_{S}$ is formally real, we have $c d_{2}\left(O_{S}\right)=+\infty$ and the above does not apply. In fact, the spectral sequence (2.2.4) does not degenerate at $E_{2}$ in this case, neither for $O_{S}$ nor for its quotient field $F$. It can be shown however that it degenerates at $E_{4}$ as well as (2.2.3), see [88, Lemma 4.3] for the latter. (For coefficients $\mathbf{Z} / 2^{s}$ with $s \geq 2$, see [83, Appendix]: this argument is detailed in Section 7 of Weibel's chapter for the real numbers. For coefficients $\mathbf{Z} / 2$, see [151]. In [159] Rognes and Weibel avoid the use of a product structure by a clever reciprocity argument.) This yields the following version of Quillen's conjecture:

Theorem 5.0.9 ([88, Th. 1]). Let $r_{1}$ be the number of real places of $F$. Then there exist homomorphisms

$$
K_{2 i-j}\left(O_{S}\right) \otimes \mathbf{Z}_{2} \stackrel{c h_{i, j}}{\longrightarrow} H_{\text {èt }}^{j}\left(O_{S}, \mathbf{Z}_{2}(i)\right) \quad(j=1,2, i \geq j)
$$

which are

(i) bijective for $2 i-j \equiv 0,1,2,7(\bmod 8)$

(ii) surjective with kernel isomorphic to $(\mathbf{Z} / 2)^{r_{1}}$ for $2 i-j \equiv 3(\bmod 8)$

(iii) injective with cokernel isomorphic to $(\mathbf{Z} / 2)^{r_{1}}$ for $2 i-j \equiv 6(\bmod 8)$.

Moreover, for $i \equiv 3(\bmod 4)$ there is an exact sequence

$$
\begin{aligned}
0 \rightarrow K_{2 i-1}\left(O_{S}\right) \otimes \mathbf{Z}_{2} \rightarrow H_{\text {èt }}^{1}\left(O_{S},\right. & \left.\mathbf{Z}_{2}(i)\right) \rightarrow(\mathbf{Z} / 2)^{r_{1}} \\
& \rightarrow K_{2 i-2}\left(O_{S}\right) \otimes \mathbf{Z}_{2} \rightarrow H_{\text {èt }}^{2}\left(O_{S}, \mathbf{Z}_{2}(i)\right) \rightarrow 0
\end{aligned}
$$


in which $\operatorname{Im}\left(H_{\text {ét }}^{1}\left(O_{S}, \mathbf{Z}_{2}(i)\right) \rightarrow(\mathbf{Z} / 2)^{r_{1}}\right)$ has 2 -rank $\rho_{i} \geq 1$ if $r_{1} \geq 1$.

The homomorphisms ch $_{i, j}$ are natural in $O_{S}$.

Remark 5.0.10. The above results hold modulo powers of an odd prime $l$ (without the non-exceptional complications) as soon as the Bloch-Kato conjecture is proven $\bmod l$. See $\S 6$ in Weibel's chapter.

For $X$ regular, Theorem 5.0.3 has been generalised by P. A. Østvær and A. Rosenschon by removing the nonexceptional hypotheses [151] : they get essentially the same statements by replacing the étale cohomological 2-dimension by the virtual étale cohomological 2-dimension. However they do not deal with singular schemes.

J. Rognes and C. Weibel [159] used Theorem 4.2.8 and the version with divisible coefficients of the spectral sequence (2.2.4) to compute much of the 2-torsion in $K_{*}\left(O_{F}\right)$ where $O_{F}$ is the ring of integers of a number field (see also [88, Cor. 3]): see Weibel's chapter in this Handbook.

Open question 5.0.11. Let be $X$ regular of finite type over $\operatorname{Spec} \mathbf{Z}[1 / m]$. Is $H^{i}(X, \mathbf{Z} / m(n))$ finite for all $i$ ?

This is false over Spec $\overline{\mathbf{Q}}$ : by Schoen [165], there exists an elliptic curve $E$ and a prime $l$ with $C H^{2}\left(E^{3}\right) / l=H^{4}\left(E^{3}, \mathbf{Z} / l(2)\right)$ infinite. I now tend to doubt whether this is true even over $\mathbf{Z}$ : see the discussion in Subsection 8.1.

\section{The picture in arithmetic geometry}

\subsection{Finite generation theorems}

A basic conjecture underlying all further conjectures is

Bass conjecture 6.1.1. a) For any scheme $X$ of finite type over $\operatorname{Spec} \mathbf{Z}$, the groups $K_{i}^{\prime}(X)$ are finitely generated.

b) For any regular scheme $X$ of finite type over $\operatorname{Spec} \mathbf{Z}$, the groups $K_{i}(X)$ are finitely generated.

By Poincaré duality for $K^{\prime}$ and $K$-theory, a) implies evidently b). But conversely, b) implies a) by the localisation exact sequence (if $X$ is of finite type over Spec $\mathbf{Z}$, its regular points form a dense open subset so we may argue by Noetherian induction).

In view of the spectral sequences (2.2.2) and (2.2.3), it is tempting to approach this conjecture via the stronger

Motivic Bass conjecture 6.1.2. a) For any scheme $X$ of finite type over Spec $\mathbf{Z}$, the groups $H_{i}^{B M}(X, \mathbf{Z}(n))$ are finitely generated.

b) For any regular scheme $X$ of finite type over $\operatorname{Spec} \mathbf{Z}$, the groups $H^{i}(X, \mathbf{Z}(n))$ are finitely generated. 
Just as before, a) $\Longleftrightarrow$ b).

I will explain in Subsection 8.1 why I now doubt that these versions of the Bass conjecture are true, and also why it does not matter too much. Nevertheless let us start with positive results:

Proposition 6.1.3. a) Conjecture 6.1.2 is true for $n \leq 1$. b) (Quillen) Conjecture 6.1.1 is true for $\operatorname{dim} X \leq 1$.

Sketch of proofs. We may reduce to $X$ regular and connected. First, a) may be deduced from a combination of

- Dirichlet's unit theorem: finite generation of units in the ring of integers of a number field, and the finiteness of the class group of such a ring.

- The Mordell-Weil theorem: for any abelian variety $A$ over a number field $K$, the group $A(K)$ is finitely generated.

- The Néron-Severi theorem: for any smooth projective variety $X$ over an algebraically closed field, the Néron-Severi group $N S(X)$ is finitely generated.

De Jong's alteration theorem also enters the proof: we skip details (see [97], and also [95, Lemma 4.1] for characteristic $p$ ).

b) Here Quillen's proofs go through a completely different path ([152], [154], [66]): homology of the general linear group. For any ring $R$, one has

$$
K_{i}(R)=\pi_{i}\left(K_{0}(R) \times B G L(R)^{+}\right) .
$$

Since $B G L(R)^{+}$is an $H$-space, by Hurewicz's theorem all $K_{i}$ are finitely generated if and only if $K_{0}(R)$ is finitely generated and all $H_{i}\left(B G L(R)^{+}, \mathbf{Z}\right)=$ $H_{i}(G L(R), \mathbf{Z})$ are finitely generated. At the time when Quillen proved the theorems, he needed to go through delicate arguments involving (in the dimension 1 case) homology of the Steinberg module. However, later stability theorems may be used to simplify the argument, except in the function field case: by van der Kallen and Maazen [98], $H_{i}(G L(R), \mathbf{Z})=H_{i}\left(G L_{N}(R), \mathbf{Z}\right)$ for $N$ large (depending on $i$ ). If $R$ is finite, this finishes the proof. If $R$ is a localised number ring, finite generation depends on a theorem of Raghunathan [156] which ultimately uses the action of $S L_{N}(R)$ on certain symmetric spaces, hence Riemannian geometry... There is a similarity with Dirichlet's proof of his unit theorem $(N=1)$.

For curves over a finite field, Quillen's proof, passing through Steinberg modules, is mainly related to the fact that semi-stable vector bundles over a curve admit moduli. It would be useful to combine this idea with the van der Kallen-Maazen stability theorem in order to simplify the proof. We shall give a completely different proof in Remark 6.8.10 3). 


\section{The Beilinson-Soulé conjecture again}

The following result was prefigured in [84]:

Theorem 6.1.4 ([87]). Conjecture 6.1.1 $\Rightarrow$ Conjecture 2.4.1.

Sketch. We shall actually sketch a proof of the slightly weaker result that Conjecture 6.1.2 $\Rightarrow$ Conjecture 2.4.1 for $X$ regular of finite type over $\mathbf{Z}[1 / 2]$. There are long exact sequences

$$
\cdots \rightarrow H^{i}\left(X, \mathbf{Z}_{(2)}(n)\right) \stackrel{2}{\longrightarrow} H^{i}\left(X, \mathbf{Z}_{(2)}(n)\right) \rightarrow H^{i}(X, \mathbf{Z} / 2(n)) \rightarrow \ldots
$$

For $i<0$, Theorem 4.2.8 + Theorem 4.2.6 $\Rightarrow H^{i}(X, \mathbf{Z} / 2(n))=0$. Since the $H^{j}\left(X, \mathbf{Z}_{(2)}(n)\right)$ are finitely generated over $\mathbf{Z}_{(2)}$, this does the proof for $i<0$. For $i=0$, we need a little more: after reducing to a finitely generated field $K$, a dyadic argument using that $K$ contains only finitely many roots of unity. With even more effort one can catch the Merkurjev-Suslin conjecture 2.4.4.

To get the actual statement of the theorem, one has to check that in the spectral sequence (2.2.3), the appropriate $E_{\infty}$ terms are uniquely 2-divisible as subquotients of motivic cohomology groups, and that then the corresponding $K$-groups are also almost uniquely divisible, hence vanish up to a group of finite exponent, and therefore the motivic groups too. This back and forth uses the degeneration of (2.2.3) up to small torsion and is a bit messy; the arguments in [84] give a good idea of it. (Note that the quasi-degeneration of the spectral sequence implies that a given $E_{\infty}$-term is equal to the corresponding $E_{2}$-term up to groups of finite exponent.) It may not be extremely interesting to make this proof completely explicit.

\section{Motivic cohomology of finite and global fields}

In this subsection we want to indicate a proof of

Theorem 6.1.5. Conjecture 6.1.2 holds for $\operatorname{dim} X \leq 1$.

Sketch. As in the proof of Proposition 6.1.3 we may restrict to $X$ regular connected. In view of Proposition 6.1.3, we may try and deduce it from Conjecture 6.1.1 via the spectral sequence (2.2.3).

If one tries the crude approach via Adams operations, one runs into the problem indicated at the end of Section 1: we only get that the groups $H^{i}(X, \mathbf{Z}(n))$ are finitely generated up to some group of finite exponent (bounded in terms of $i$ and $n$ ). We are going to get by by granting the Beilinson-Lichtenbaum conjecture 4.2.4. The main point is:

Lemma 6.1.6. The Beilinson-Soulé conjecture 2.4.1 is true for $\operatorname{dim} X \leq 1$; moreover $H^{i}(X, \mathbf{Z}(n))=0$ for $i \geq \operatorname{dim} X+2$ (up to a finite 2-group if the function field of $X$ is formally real). 
There are three very different proofs of this lemma. The first combines the rank computations of Borel [24] with the results of Soulé [172, 173], cf. [77, p. 327, Ex. 3]. The second uses the proof of the rank conjecture for number fields (the rank filtration is opposite to the gamma filtration) by Borel and Yang [27]. The third is to apply Theorem 6.1.4 in this special case: see [88, proof of Th. 4.1].

Given Lemma 6.1.6, (2.2.3) degenerates at $E_{2}$ for dimension reasons, except in the formally real case. When it degenerates at $E_{2}$ the finite generation conclusion is immediate; in the formally real case one gets relationships between $K$-theory and motivic cohomology similar to those of Theorem 5.0.9 and the conclusion follows again.

To get this finite generation result for motivic cohomology, we have used a very circuitous and quite mathematically expensive route: Quillen's finite generation theorems for $K$-theory (involving the homology of $G L_{n}$ and Riemannian geometry), the Bloch-Lichtenbaum spectral sequence and finally the Bloch-Kato conjecture! In characteristic 0 this seems to be the only available route at the moment. In characteristic $p$, however, we shall see in Remark 6.8.10 3) that Frobenius provides a shortcut allowing us to avoid the passage through $K$-theory.

\subsection{Ranks, torsion and zeta functions}

The primeval formula in this subject is certainly Dedekind's analytic class number formula: let $K$ be a number field, $\zeta_{K}$ its Dedekind zeta function, $\left(r_{1}, r_{2}\right)$ its signature, $h$ its class number, $w$ the number of its roots of unity and $R$ its regulator. Then

$$
\lim _{s \rightarrow 0} s^{-r_{1}-r_{2}+1} \zeta_{K}(s)=-\frac{h R}{w} .
$$

So we recover analytically the rank $r_{1}+r_{2}-1$ of the units $O_{K}^{*}$ as well as a number involving $h, R$ and $w$. Up to the rational number $h / w$, the special value of $\zeta_{K}(s)$ at $s=0$ is the regulator. Deligne, Lichtenbaum, Soulé and Beilinson have formulated conjectures generalising this formula. These conjectures really are for two very different types of zeta or $L$-functions:

- The zeta function of an arithmetic scheme (Lichtenbaum, Soulé).

- The "Hasse-Weil" $L$-functions associated to $H^{i}$ of a smooth projective variety over a number field $K$, or more generally to a $K$-motive for absolute Hodge cycles (Deligne, Beilinson, Bloch-Kato...)

They have shaped the development of algebraic $K$-theory and later motivic cohomology and the theory of motives ever since they were formulated. Here I am only going to discuss the first case: the second one is much harder to even state and completely beyond the scope of these notes. 


\subsection{Soulé's conjecture}

Lichtenbaum formulated very precise conjectures, at least in special cases, while Soulé formulated a general conjecture but only for orders of poles. Let me start with this one. Recall that an arithmetic scheme is a scheme of finite type over $\mathbf{Z}$. If $X$ is an arithmetic scheme, its zeta function ${ }^{9}$ is

$$
\zeta(X, s)=\prod_{x \in X_{(0)}}\left(1-N(x)^{-s}\right)^{-1}
$$

where $X_{(0)}$ is the set of closed points of $X$ and, for $x \in X_{(0)}, N(x)=|\kappa(x)|$, the cardinality of the residue field at $x$. This formal expression has some obvious properties:

1. $\zeta(X, s)$ only depends on the reduced structure of $X$.

2. If $Z$ is closed in $X$ with open complement $U$, then

$$
\zeta(X, s)=\zeta(U, s) \zeta(Z, s) .
$$

3.

$$
\zeta\left(X \times \mathbf{A}^{1}, s\right)=\zeta(X, s-1) .
$$

4. If $f: X \rightarrow Y$ is a morphism, then

$$
\zeta(X, s)=\prod_{\left.y \in Y_{0}\right)} \zeta\left(X_{y}, s\right)
$$

where $X_{y}$ is the fibre of $f$ at $y$.

Using this, one easily proves that $\zeta(X, s)$ converges absolutely for $\operatorname{Re}(s)>$ $\operatorname{dim} X$ by reducing to Riemann's zeta function (see [168, Proof of Theorem 1] for details), hence is analytic in this domain as a Dirichlet series. It is conjectured to have a meromorphic continuation to the whole complex plane: this is known at least in the half-plane $\operatorname{Re}(s)>\operatorname{dim} X-1 / 2$ [168, Th. 2].

Finally, if $X$ is defined over a finite field $k$ with $q$ elements, then one has the famous formula (Grothendieck-Artin-Verdier):

$$
\zeta(X, s)=\prod_{i=0}^{2 d} \operatorname{det}\left(1-F_{X} q^{-s} \mid H_{c}^{i}\left(\bar{X}, \mathbf{Q}_{l}\right)\right)^{(-1)^{i+1}}
$$

where $H_{c}^{*}\left(\bar{X}, \mathbf{Q}_{l}\right)$ is the $\mathbf{Q}_{l}$-adic cohomology with compact supports of the geometric fibre $\bar{X}$ [SGA 5 , Exp. XV]. In particular $\zeta(X, s)$ is a rational function in $q^{-s}$ (a result originally proven by Dwork [42]) and the meromorphic continuation is obvious.

If $X=\operatorname{Spec} O_{K}$ for a number field $K$, we recover the Dedekind zeta function of $K$.

\footnotetext{
${ }^{9}$ This notion goes back to Artin, Hasse and Weil. To the best of my knowledge, the place where it is first defined in this generality is Serre [167].
} 
Soulé conjecture 6.3.1 ([174, Conj.2.2]). For any $n \in \mathbf{Z}$, we have

$$
\operatorname{ord}_{s=n} \zeta(X, s)=\sum_{i \in \mathbf{Z}}(-1)^{i+1} \operatorname{dim}_{\mathbf{Q}} K_{i}^{\prime}(X)_{(n)}
$$

where $K_{i}^{\prime}(X)_{(n)}$ is the part of weight $n$ of $K_{i}^{\prime}(X)$ under the homological Adams operations.

Remarks 6.3.2. 1) This is a conjecture built over conjectures! First, it presupposes the meromorphic continuation of $\zeta(X, s)$. Then, implicitely, the dimensions involved in this formula are finite and almost all 0 : this would be a consequence of Conjecture 6.1.1, via Theorem 6.1.4.

2) Using (2.2.8), we may now rewrite the right hand side as

$$
\sum_{i \in \mathbf{Z}}(-1)^{i+1} \operatorname{dim}_{\mathbf{Q}} H_{2 n-i}^{B M}(X, \mathbf{Q}(n))=\sum_{j \in \mathbf{Z}}(-1)^{j+1} \operatorname{dim}_{\mathbf{Q}} H_{j}^{B M}(X, \mathbf{Q}(n))
$$

which looks much more like an Euler-Poincaré characteristic.

For a conjecture on the special values of this zeta function, see Theorem 6.8.11.

Example 6.3.3. Let $X=\operatorname{Spec} O_{K}$, where $K$ is a number field. It is known that $K_{i}(X)_{(n)}=K_{i}(X)^{(1-n)}=0$ for $i \neq 2(1-n)-1=1-2 n$, except that $K_{i}(X)^{(0)}=0$ for $i \neq 0$; moreover $K_{0}(X)^{(0)}=K_{0}(X) \otimes \mathbf{Q}$ and $K_{1-2 n}(X)^{(1-n)}=K_{1-2 n}(X) \otimes \mathbf{Q}$. Hence the conjecture reads, replacing $n$ by $1-n$ :

$$
\operatorname{ord}_{s=1-n} \zeta(X, s)= \begin{cases}-\operatorname{dim}_{\mathbf{Q}} K_{0}(X) \otimes \mathbf{Q} & \text { for } n=0 \\ \operatorname{dim}_{\mathbf{Q}} K_{2 n-1}(X) \otimes \mathbf{Q} & \text { for } n>0 .\end{cases}
$$

For $n=0$, this says that $\zeta(X, s)$ has a pole of order 1 at $s=1$, which is classical. For $n=1$, it follows from (6.2.1). For $n>1$, it is easy to compute the left hand side via the functional equation: one finds

$$
g_{n}:=\operatorname{ord}_{s=1-n} \zeta(X, s)= \begin{cases}r_{1}+r_{2} & \text { if } n \text { is odd } \\ r_{2} & \text { if } n \text { is even. }\end{cases}
$$

It is a theorem of Borel [24] that the right hand side has the same value, so that (6.3.5) is true (of course, Soulé's conjecture was only formulated much later); this prompted Lichtenbaum's conjecture (or question) 6.4.1 below, which in turn prompted further work of Borel in this direction [25], see Theorem 6.4.2.

Let us toy with the Soulé conjecture as we toyed with the Beilinson-Soulé conjecture. From (6.3.1) and (2.2.1) (or the easier localisation theorem of Quillen for $K^{\prime}$-theory), one deduces that if $X=U \cup Z$ and the conjecture is true for two among $X, U, Z$, then it is true for the third. From this follows easily: 
Lemma 6.3.4. The following conditions are equivalent:

(i) Conjecture 6.3.1 is true for all $X$.

(ii) Conjecture 6.3.1 is true for all $X$ affine and regular.

(iii) Conjecture 6.3.1 is true for all $X$ projective over $\mathbf{Z}$.

One would like to refine this lemma further, reducing to $X$ as in Lemma $2.4 .3 \mathrm{c}$ ) (or d) if we restrict to $X \mathrm{~s}$ of positive characteristic). Unfortunately I don't see how to do this: unlike an abelian group, a number does not have direct summands! This reduction will work however if we know some strong form of resolution of singularities (e.g. for $\operatorname{dim} X \leq 2$, by Abyankhar). This approach is probably too crude, see Theorem 6.7.5.

Soulé's conjecture is true for $n>d=\operatorname{dim} X$ because both sides of the equality are then 0 . For $n=d$ it is true by [168, Th. 6]. For $n=d-1$ and $X$ regular and irreducible, it was formulated by Tate in [188] and implies the Birch-Swinnerton-Dyer conjecture (in fact, is equivalent to it under some strong enough form of resolution of singularities, see above); more generally, it is compatible with the Beilinson conjectures in a suitable sense. For details on all this, see Soulé [174, 2.3 and 4.1]. Finally, it is equivalent to a part of Lictenbaum's second conjecture if $X$ is smooth projective over a finite field, see Subsection 6.6.

We shall now state the two conjectures of Lichtenbaum on special values of zeta functions: the first concerns Dedekind zeta functions and the second those of smooth projective varieties over a finite field.

\subsection{Lichtenbaum's first conjecture: rings of algebraic integers}

(See Goncharov's chapter for many more details on this subsection, including the relationship with polylogarithms.)

To state this conjecture, recall that $K_{2 n}\left(O_{K}\right)$ is finite because it is finitely generated (Proposition 6.1.3) and has rank 0 (Borel [24]). Next, Borel defined a regulator

$$
\rho_{n}: K_{2 n-1}\left(O_{K}\right) \otimes \mathbf{R} \rightarrow \mathbf{R}^{g_{n}}
$$

which is an isomorphism by [24]. Let $R_{n}(K)$ be the absolute value of the determinant of $\rho_{n}$ with respect of a basis of $K_{2 n-1}\left(O_{K}\right) /$ tors and the canonical basis of $\mathbf{R}^{g_{n}}$. Lichtenbaum asks prudently:

Conjecture 6.4.1 (Lichtenbaum [123, Question 4.2]). When is it true that

$$
\lim _{s \rightarrow 1-n}(s+n-1)^{-g_{n}} \zeta_{K}(s)= \pm \frac{\left|K_{2 n-2}\left(O_{K}\right)\right|}{\left|K_{2 n-1}\left(O_{K}\right)_{\text {tors }}\right|} R_{n}(K) ?
$$

Note that the sign is not mysterious at all: it is easy to get as follows. If we restrict $\zeta_{K}(s)$ to $s$ real, it takes real values. For $s>0$ it is positive. Since

it has a single pole at $s=1$, it is negative for $s<1$ in the neighbourhood of $s=1$. Between $s=0$ and $s=1$, its only possible zero is for $s=1 / 2$. But 
the functional equation shows that this possible zero has even order (I am indebted to Pierre Colmez for this trick). Therefore its value for $s>0$ near 0 is still negative and the sign at $s=0$ is -1 . Then it in known that the only zeroes or poles are at negative integers, and the above reasoning gives the sign in (6.4.1) immediately. One finds

$$
(-1)^{g_{n-1}+g_{n-2}+\cdots+g_{1}+1}= \begin{cases}(-1)^{\frac{n}{2} r_{1}+r_{2}} & \text { if } n \text { is even } \\ (-1)^{\frac{n-1}{2} r_{1}} & \text { if } n \text { is odd }>1 .\end{cases}
$$

This computation also appears in Kolster [111] (using the functional equation).

In [25], Borel gave the following partial answer:

Theorem 6.4.2.

$$
\lim _{s \rightarrow 1-n}(s+n-1)^{-g_{n}} \zeta_{K}(s)=R_{n}(K)
$$

up to a nonzero rational number.

Here are some comments on Conjecture 6.4.1. Besides (6.2.1) and Borel's computation of the ranks of $K$-groups in [24], it was inspired by an earlier conjecture of Birch and Tate (the case $n=2$ ) when $K$ is totally real [17], [190] and by a conjecture of Serre [170, p. 164] that, still for $K$ totally real and $n$ even, $\zeta_{K}(1-n)\left|H^{0}(K, \mathbf{Q} / \mathbf{Z}(n))\right|$ should be an integer; this conjecture was proven later by Deligne-Ribet [40]. This case is much simpler because then $g_{n}=0$ and it had been proven long ago by Siegel that the left hand side of (6.4.1) was a rational number. In this special case, Lichtenbaum initially conjectured the following equality:

$$
\zeta_{K}(1-n)= \pm \prod_{l \text { prime }} \frac{\left|H^{1}\left(O_{K}[1 / l], \mathbf{Q}_{l} / \mathbf{Z}_{l}(n)\right)\right|}{\left|H^{0}\left(O_{K}[1 / l], \mathbf{Q}_{l} / \mathbf{Z}_{l}(n)\right)\right|} .
$$

Under this form, the conjecture was proven by Mazur-Wiles for $K$ abelian over $\mathbf{Q}$ [130], and then by Wiles in general, except perhaps for $l=2$ [211], as a consequence of their proofs of Iwasawa's Main Conjecture. Still in this special case, Quillen's conjectures (5.0.7) and (5.0.8) prompted the $K$-theoretic formulation (6.4.1), up to a power of 2 since (5.0.7) and (5.0.8) were formulated only for $l$ odd and computations showed that the 2-primary part of the formula was false. This is now explained, for example, by Theorem 5.0.9: the correct formula, still in the case where $K$ is totally real and $n$ is even, is ( $c f$. [88, Cor. 1], [159])

$$
\zeta_{K}(1-n)= \pm 2^{r_{1}} \frac{\left|K_{2 n-2}\left(O_{K}\right)\right|}{\left|K_{2 n-1}\left(O_{K}\right)\right|}= \pm \frac{\left|H^{2}\left(O_{K}, \mathbf{Z}(n)\right)\right|}{\left|H^{1}\left(O_{K}, \mathbf{Z}(n)\right)\right|} .
$$

We could say that this conjecture is essentially proven now if one believes that the proof of the Bloch-Kato conjecture (for Milnor's $K$-theory) is complete. 
How about the general conjecture? First there was an issue on the correct normalisation of the Borel regulator, as Borel's original definition does not give Theorem 6.4.2, but the same formula with the right hand side multiplied by $\pi^{g_{n}}$. The normalisation issue is basically accounted for by the difference between the Hodge structures $\mathbf{Z}$ and $\mathbf{Z}(1)=2 \pi i \mathbf{Z}$ : we refer to [30, Ch. 9] for a very clear discussion (see also [26]). Then Beilinson formulated his general conjectures which should have Borel's theorem as a special case: there was therefore the issue of comparing the Borel and the Beilinson regulators. ${ }^{10}$ This was done by Beilinson himself up to a nonzero rational number (see [157]), and finally Burgos [30] showed that the Beilinson and Borel regulator maps differ by a factor 2 , hence the corresponding determinants differ by $2^{g_{n}}$.

Let me give what I believe is the correct formulation in terms of motivic cohomology and a version of Beilinson's regulator (see also for example [110, 111]). This will be the only allusion to Beilinson's point of view in this survey. We define $H^{i}\left(O_{K}, \mathbf{Z}(n)\right)$ as Levine does in [119] and [121], using a suitable version of Bloch's cycle complexes for schemes over $\mathbf{Z}$. Then the construction of a motivic cycle class map yields "regulator" maps to Deligne's cohomology (see $\S 6.1$ in Levine's chapter; note that $H^{1}\left(O_{K}, \mathbf{Q}(n)\right) \stackrel{\sim}{\longrightarrow} H^{1}(K, \mathbf{Q}(n))$ for $n \geq 2$ and that the regulator is just Dirichlet's regulator for $n=1)$

$$
\rho_{n}^{\prime}: H^{1}\left(O_{K}, \mathbf{Z}(n)\right) \otimes \mathbf{R} \rightarrow H_{D}^{1}\left(O_{K} \otimes \mathbf{z} \mathbf{R}, \mathbf{R}(n)\right)
$$

which can be compared to Beilinson's regulator, the latter being essentially a Chern character. The Lichtenbaum conjecture should then read

$$
\lim _{s \rightarrow 1-n}(s+n)^{-g_{n}} \zeta_{K}(s)=\varepsilon_{n} \frac{\left|H^{2}\left(O_{K}, \mathbf{Z}(n)\right)\right|}{\left|H^{1}\left(O_{K}, \mathbf{Z}(n)\right)_{\text {tors }}\right|} R_{n}^{\prime}(K)
$$

where $R_{n}^{\prime}(K)$ is the absolute value of the determinant of $\rho_{n}^{\prime}$ with respect to integral bases and $\varepsilon_{n}$ is as in (6.4.2).

The best formulation would be in terms of étale motivic cohomology with compact supports à la Kato-Milne [136, p. 203], but this would lead us too far. (Lichtenbaum is currently working on a conjectural formula involving cohomology groups defined by means of the Weil groups, in the spirit of his Weil-étale topology in characteristic $p$ which we shall explain in Subsection 6.8.)

As for the general case of the Lichtenbaum conjecture, it is now proven with the same caveat (Bloch-Kato conjecture) for $K$ abelian over $\mathbf{Q}$, by the work of Fleckinger-Kolster-Nguyen Quang Do [47] (see also [15] and [16, appendix]). For nonabelian $K$ we are still far from a proof.

Note that, if one is only interested in totally real $K$ and even $n$, one may reformulate Conjecture 6.4.1 purely in terms of étale cohomology, and if one

$\overline{10}$ The great superiorities of the Beilinson regulator on the Borel regulator are its conceptual definition, its functoriality and its computability in certain cases. On the other hand, no proof of Theorem 6.4.2 directly in terms of the Beilinson regulator is known at present. 
is only interested in Theorem 6.4.2 one may reformulate things in terms of the homology of $G L_{n}\left(O_{K}\right)$. In both cases one can get rid of algebraic $K$ theory and motivic cohomology. However, if one wants the general case, there is no way to avoid them. This encapsulates the beauty and the depth of this conjecture!

Remark 6.4.3. In the sequel we shall amply discuss varieties over finite fields. Let us make here a few comments on the 1-dimensional case. Let $X$ be a smooth projective curve over $\mathbf{F}_{p}$. By the already mentioned theorem of Quillen [66], the groups $K_{i}(X)$ are finitely generated. On the other hand, their rank was computed by the work of Harder [73]: for $i>0$ it is 0 , hence $K_{i}(X)$ is finite. Harder computes the rank of the homology of $S L_{n}(A)$, where $A$ is the coordinate ring of an affine open subset of $X$, very much in the style of Borel [24], hence using Riemannian geometry.

There are two completely different proofs of this rank computation. The first one is due to Soulé $[175,2.3 .4]$ and uses motivic methods: see Subsection 7.2 below. The second one uses the Milnor conjecture (Theorem 4.2.8): by the spectral sequence (2.2.3) (or the isomorphism (2.2.6)) it is enough to show that $H^{i}(X, \mathbf{Q}(n))=0$ for $i \neq 2 n$. This can be done as for the proof of Theorem 6.1.4. This argument relies on knowing the finite generation of the $K_{i}(X)$ while Harder's and Soulé's proofs do not. On the other hand, we shall get the finite generation of $H^{i}(X, \mathbf{Z}(n))$ directly in Section 7, without appealing to Quillen's theorem but using the Bloch-Kato conjecture.

\subsection{The Tate, Beilinson and Parshin conjectures}

For the rest of this section, $k$ is a finite field and $X$ is a smooth projective $k$-variety. We also give ourselves a nonnegative integer $n$. Before introducing the second conjecture of Lichtenbaum, it is appropriate to recall two closely related conjectures. The first one is the famous Tate conjecture:

Tate conjecture 6.5.1. $\operatorname{ord}_{s=n} \zeta(X, s)=-\operatorname{dim}_{\mathbf{Q}} A_{\text {num }}^{n}(X, \mathbf{Q})$.

The second one, due to Beilinson, is a special case of his conjectures on filtrations on Chow groups [81].

Beilinson conjecture 6.5.2. $A_{\text {rat }}^{n}(X, \mathbf{Q})=A_{\text {num }}^{n}(X, \mathbf{Q})$.

There is a third related conjecture, due to Beilinson and Parshin:

Beilinson-Parshin conjecture 6.5.3. $K_{i}(X)$ is torsion for $i>0$.

In view of (2.2.7), the Beilinson-Parshin conjecture may be reformulated in terms of motivic cohomology as follows: $H^{i}(X, \mathbf{Q}(n))=0$ for $i \neq 2 n$. In particular, this conjecture is a strong reinforcement of the Beilinson-Soule conjecture for schemes of characteristic $p$ (compare Lemma 2.4.3).

Geisser has proven:

Theorem 6.5.4 ([56, Th. 3.3]). Conjecture 6.5.1 + Conjecture 6.5.2 $\Rightarrow$ Conjecture 6.5.3. 
Similarly, the Bass conjecture 6.1.1 implies Conjecture 6.5.3, just as it implies Conjecture 2.4.1 [87].

Let us compare these conjectures with Soulé's conjecture 6.3.1 restricted to smooth projective varieties over $\mathbf{F}_{p}$. Using the functional equation, Conjecture 6.5.1 may be reformulated as follows: $\operatorname{ord}_{s=n} \zeta(X, s)=-\operatorname{dim}_{\mathbf{Q}} A_{n}^{\text {num }}(X, \mathbf{Q})$. On the other hand, Conjecture 6.3.1 predicts that the value of the left hand side should be $\sum_{i \in \mathbf{Z}}(-1)^{i+1} \operatorname{dim}_{\mathbf{Q}} H_{i}^{B M}(X, \mathbf{Q}(n)$ ) (see Remark 6.3.2 $2)$ ). Under Conjecture 6.5.3, this reduces to $-\operatorname{dim}_{\mathbf{Q}} H_{2 n}^{B M}(X, \mathbf{Q}(n))=$ $-\operatorname{dim}_{\mathbf{Q}} C H_{n}(X) \otimes \mathbf{Q}$. Hence, assuming the Parshin conjecture, among the Soule, the Tate and the Beilinson conjecture, any two imply the third. In particular, the Tate conjecture plus the Beilinson conjecture imply the Soule conjecture for smooth projective varieties - but see in fact Theorem 6.7.5 below. Alternatively, we may replace the use of the functional equation by the observation that $\operatorname{dim}_{\mathbf{Q}} A_{\text {num }}^{n}(X, \mathbf{Q})=\operatorname{dim}_{\mathbf{Q}} A_{\text {num }}^{d-n}(X, \mathbf{Q})$, where $d=\operatorname{dim} X$.

The Tate conjecture is known in codimension 1 for abelian varieties, by Tate's theorem [189]; it is trivial in dimension 0. Besides this it is known in many scattered cases, all being either abelian varieties or varieties "of abelian type", see examples 7.1.2 below. In particular, Soulé deduced it from [189] for products of 3 curves by a very simple motivic argument, and then for all varieties of abelian type of dimension $\leq 3$ (in a slightly restricted sense compared to the one of [95], see Example 7.1.2 1)) by a dévissage argument from the former case [175, Th. 4 (i)].

The Beilinson conjecture is trivial in codimension 1 ; in dimension 0 it is true for any variety by a theorem of Kato and Saito [102]. Soulé proved it in the same cases as the Tate conjecture (loc. cit.), and in particular his conjecture 6.3.1 is true for this type of varieties. Besides this, it was unknown except for trivial cases like projective homogeneous varieties until Theorem 7.1.3 below, which proves new cases of it.

Finally, let us give a consequence of Conjecture 6.5.3 for fields, using de Jong's alteration theorem (cf. [56, Th. 3.4]):

Lemma 6.5.5. If Conjecture 6.5.3 holds for all smooth projective varieties over $\mathbf{F}_{p}$, then for any field $K$ of characteristic $p$ and any $n \geq 0$,

(i) $H^{i}(K, \mathbf{Q}(n))=0$ for $i \neq n$.

(ii) $K_{n}^{M}(K)$ is torsion as soon as $n>\operatorname{trdeg}\left(K / \mathbf{F}_{p}\right)$ (Bass-Tate conjecture).

The proof goes exactly as in that of Lemma 2.4.3 (recall that $H^{i}(K, \mathbf{Z}(n)$ ) $=0$ for $i>n$ anyway). As for the consequence on Milnor's $K$-theory, one uses Theorem 2.2.1 and the fact that $H^{2 n}(X, \mathbf{Z}(n))=C H^{n}(X)=0$ for $n>\operatorname{dim} X$.

\subsection{Lichtenbaum's second conjecture: varieties over finite fields}

This conjecture, which appears in [124], was formulated in two steps, in terms of a not yet constructed "arithmetic cohomology theory", later rechristened "motivic cohomology". It is important to notice that Lichtenbaum formulated it for the étale hypercohomology of certain complexes. Here it is: 
1. $H_{\text {ét }}^{i}(X, \mathbf{Z}(n))=0$ for $i$ large.

2. $H_{\text {êt }}^{2 n}(X, \mathbf{Z}(n))$ is a finitely generated abelian group.

3. $H_{\text {ét }}^{i}(X, \mathbf{Z}(n))$ is finite for $i \neq 2 n, 2 n+2,0$ for $i \leq 0$ when $n>0$.

4. $H_{\mathrm{et}}^{2 d+2}(X, \mathbf{Z}(d))$ is canonically isomorphic to $\mathbf{Q} / \mathbf{Z}$, where $d=\operatorname{dim} X$.

5. The pairing

$$
H_{\text {êt }}^{i}(X, \mathbf{Z}(n)) \times H_{\text {ét }}^{2 d+2-i}(X, \mathbf{Z}(d-n)) \rightarrow H_{\text {ét }}^{2 d+2}(X, \mathbf{Z}(d)) \stackrel{\sim}{\longrightarrow} \mathbf{Q} / \mathbf{Z}
$$

is "perfect" in the sense that it defines a perfect duality of finite groups for $i \neq 2 n$ and a perfect duality between a finitely generated group and a group of finite cotype pour $i=2 n$. In particular, $\operatorname{rg} H_{\text {ét }}^{2 d}(X, \mathbf{Z}(d))=1$.

6 . The groups $H_{\text {ét }}^{2 n}(X, \mathbf{Z}(n))$ and $H_{\text {ét }}^{2 d-2 n}(X, \mathbf{Z}(d-n))$ have the same rank $m(n)$.

7. $m(n)$ is the order of the pole of $\zeta(X, s)$ at $s=n$.

8. $\lim _{s \rightarrow n}\left(1-q^{n-s}\right)^{m(n)} \zeta(X, s)= \pm q^{\chi\left(X, \mathcal{O}_{X}, n\right)} \chi(X, \mathbf{Z}(n))$, with

$$
\begin{aligned}
& \chi(X, \mathbf{Z}(n))= \\
& \prod_{i \neq 2 n, 2 n+2}\left|H_{\text {èt }}^{i}(X, \mathbf{Z}(n))\right|^{(-1)^{i}} \cdot \frac{\left|H_{\text {ét }}^{2 n}(X, \mathbf{Z}(n))_{\text {tors }}\right|\left|H_{\text {ét }}^{2 n+2}(X, \mathbf{Z}(n))_{\text {cotors }}\right|}{R_{n}(X)}
\end{aligned}
$$

where $R_{n}(X)$ is the absolute value of the determinant of the pairing

$$
\begin{aligned}
H_{\text {ét }}^{2 n}(X, \mathbf{Z}(n)) / \text { tors } \times H_{\text {ét }}^{2 d-2 n}(X, \mathbf{Z}(d-n)) / \text { tors } & \\
& \rightarrow H_{\text {ét }}^{2 d}(X, \mathbf{Z}(d)) / \text { tors } \stackrel{\sim}{\longrightarrow} \mathbf{Z}
\end{aligned}
$$

relatively to arbitrary bases of $\frac{H_{\mathrm{et}}^{2 n}(X, \mathbf{Z}(n))}{\text { tors }}$ and $\frac{H_{\mathrm{et}}^{2 d-2 n}(X, \mathbf{Z}(d-n))}{\text { tors }}$, and

$$
\chi\left(X, \mathcal{O}_{X}, n\right)=\sum_{\substack{0 \leq i \leq n \\ 0 \leq j \leq d}}(-1)^{i+j}(n-i) h_{i j}, \quad h_{i j}=\operatorname{dim} H^{j}\left(X, \Omega^{i}\right) .
$$

Let us examine these predictions in terms of the present state of knowledge. Statement 1 is known: for $i>2 n+1, H_{\text {ét }}^{i-1}(X, \mathbf{Q} / \mathbf{Z}(n)) \stackrel{\sim}{\longrightarrow} H_{\text {ét }}^{i}(X, \mathbf{Z}(n))$ by Theorem 4.2.1 and the discussion before Theorem 2.2.1; but $c d(X)=$ $2 d+1$ since a finite field has étale cohomological dimension 1 . So we may take $i>2 d+2$. Similarly, Statement 4 is known, as well as the fact that $\operatorname{rk} H_{\text {ét }}^{2 d}(X, \mathbf{Z}(d))=1$.

For the other statements, the following remarks are in order. Statement 6 is a formal consequence of the part of 5 which predicts a nondegenerate pairing between the two groups. In view of Theorem 4.2.1 and the fact that $H^{2 n}(X, \mathbf{Z}(n))=C H^{n}(X)$, Statement 7 follows from the conjunction of Conjectures 6.5.1 and 6.5.2; given 3 and 5 it is equivalent to Soulé's conjecture 6.3.1. Finally, statements 2,3 and 5 are striking in that they predict finite generation properties of étale motivic cohomology, but in a rather scattered way. This will be corrected (by an idea of Lichtenbaum!) in Subsection 6.8. 


\subsection{Motivic reformulation of the Tate and Beilinson conjectures}

One major point of this whole story is that Conjectures 6.5.1 and 6.5.2 really have to be considered together. Then they have a very nice and very powerful reformulation: this was the subject of [90]. I wrote it using Voevodsky's version of motivic cohomology, which made a rather simple construction but necessitated some undesirable assumptions on resolution of singularities in characteristic $p$. The version with Bloch's higher Chow groups, developed in [93], involves more technicality but is free of resolution of singularities assumptions. Let me explain it now.

For $l \neq p$, define

$$
\mathbf{Z}_{l}(n)^{c}=R \lim _{\longleftarrow} \mu_{l^{s}}^{\otimes n} .
$$

This is an object of $D^{+}\left(\left(S m / \mathbf{F}_{p}\right)\right.$ ét $)$, whose hypercohomology computes Jannsen's continuous étale cohomology $H_{\text {cont }}^{*}\left(X, \mathbf{Z}_{l}(n)\right)$ [76] for smooth varieties $X$ over $\mathbf{F}_{p}$. Naturally, by Deligne's finiteness theorem for étale cohomology [SGA 4 1/2, Th. Finitude], we have

$$
H_{\text {cont }}^{i}\left(X, \mathbf{Z}_{l}(n)\right)=\lim _{\longleftarrow} H_{\text {ét }}^{i}\left(X, \mu_{l^{s}}^{\otimes n}\right)
$$

but this is a theorem rather than a definition. In any case, the quasiisomorphisms of Theorem 4.1.1 a) can now be assembled into a morphism in $D\left(\left(S m / \mathbf{F}_{p}\right)\right.$ ét $)[93, \S 1.4]$ :

$$
\alpha^{*} \mathbf{Z}(n) \otimes \mathbf{Z}_{l} \rightarrow \mathbf{Z}_{l}(n)^{c} .
$$

If one is looking for a quasi-isomorphism then this morphism is not yet quite right: for example, for $n=0$ the left hand side is $\mathbf{Z}_{l}$ while the isomorphisms $H_{\text {cont }}^{1}\left(\mathbf{F}_{q}, \mathbf{Z}_{l}\right) \stackrel{\sim}{\longrightarrow} \mathbf{Z}_{l}$ for all $q$ yield $\mathcal{H}^{1}\left(\mathbf{Z}_{l}(0)^{c}\right) \stackrel{\sim}{\longrightarrow} \mathbf{Q}_{l}[90, \S 4$ and $\mathrm{Th}$. 6.3]. Using cup-product, let us perform the minimal modification correcting this: we get a morphism

$$
\alpha^{*} \mathbf{Z}(n) \stackrel{L}{\otimes} \mathbf{Z}_{l}(0)^{c} \rightarrow \mathbf{Z}_{l}(n)^{c} .
$$

Theorem 6.7.1 ([93, Th. 3.4]). The following statements are equivalent:

(i) Conjectures 6.5.1 and 6.5.2 are true for all $X, n$.

(ii) (6.7.1) is an isomorphism for any $n$.

(iii) $\mathbf{Z}_{l}(n)^{c}$ is malleable for any $n>0$ (see $p$. 27).

For $l=p$ one can define a morphism analogous to (6.7.1), using instead of $\mathbf{Z}_{l}(n)^{c}$ the object

$$
\mathbf{Z}_{p}(n)^{c}:=R \lim _{\longleftarrow} \nu_{s}(n)[-n]
$$

where $\nu_{s}(n)$ is the sheaf of logarithmic de Rham-Witt differential forms:

$$
\alpha^{*} \mathbf{Z}(n) \stackrel{L}{\otimes} \mathbf{Z}_{p}(0)^{c} \rightarrow \mathbf{Z}_{p}(n)^{c} .
$$

see $[95, \S 3.5]$. Then an equivalent condition to the above is ( $c f .[95, \S 3.6],[58])$ : 
(ii) bis (6.7.2) yields an isomorphism on the hypercohomology of any smooth projective $X$.

Note that (i) involves only algebraic cycles, (iii) involves only cohomology and (ii) is a comparison between them. Also, (i) does not involve $l$, hence (ii) and (iii) are independent of $l$.

In fact, in $[90, \S 4]$ we construct a complex of length 1 of $G_{\mathbf{F}_{p}}$-modules $\mathbf{Z}^{c}$ such that for all $l$ (including $l=p$ ) there is a canonical isomorphism

$$
\mathbf{Z}_{l}(0)^{c} \simeq \pi^{*} \mathbf{Z}^{c} \otimes \mathbf{Z}_{l}
$$

where $\pi$ is the projection of the big étale site of $\operatorname{Spec} \mathbf{F}_{p}$ onto its small étale site (ibid., Th. $4.6 \mathrm{~b}$ ) and 6.3). So, strikingly, (ii) predicts the existence of a canonical integral structure on arithmetic $l$-adic cohomology, independent of $l$. (One should not confuse this prediction with the "independence of $l$ " conjectures for geometric l-adic cohomology.)

Sketch. The equivalence between (ii) and (iii) follows from Theorem 4.2.7: the fact that Condition 2 in it is satisfied follows from the results of [94]. The proof of the equivalence between (i) and (ii) is not really difficult: first, by Theorem 4.1.1 a), (ii) $\otimes^{L} \mathbf{Z} / l^{\nu}$ is true, so (ii) and (ii) $\otimes \mathbf{Q}$ are equivalent. Using de Jong, we get as in Lemma 2.4.3 that (ii) $\otimes \mathbf{Q}$ holds if and only if it holds for every smooth projective variety $X$. Then one examines the two sides of the maps

$$
H_{\text {ét }}^{i}\left(X, \mathbf{Q}(n) \otimes \mathbf{Q}_{l}(0)^{c}\right) \rightarrow H_{\text {cont }}^{i}\left(X, \mathbf{Q}_{l}(n)\right)
$$

and one deduces via the "Riemann hypothesis" (Weil conjecture) and some of the folklore in [191] that isomorphism for all $i$ and $n$ is equivalent to the conjunction of conjectures 6.5.1, 6.5.2 and 6.5.3. One concludes by Theorem 6.5.4. See [93] for details.

Definition 6.7.2 (Tate-Beilinson conjecture). For simplicity, we call the equivalent conjectures of Theorem 6.7.1 the Tate-Beilinson conjecture.

\section{Some consequences}

Besides being clearly of a motivic nature, the main point of the Tate-Beilinson conjecture under the form (ii) in Theorem 6.7.1 is that it allows one to pass easily from smooth projective varieties to general smooth varieties, or even to arbitrary schemes of finite type over $\mathbf{F}_{p}$. It has remarkable consequences: one could say that it implies almost everything that one expects for varieties over finite fields. We have already seen that it implies the Beilinson-Parshin conjecture (via Geisser's Theorem 6.5.4), hence the Beilinson-Soulé conjecture in characteristic $p$. But there is much more. Let us first give some motivic consequences.

By [138, Remark 2.7 and Theorem 2.49], Conjecture 6.5.1 implies: 
- For any finite field $k, \operatorname{Mot}_{\text {num }}(k, \mathbf{Q})$ is generated by motives of abelian varieties and Artin motives.

- Every mixed motive over a finite field is a direct sum of pure motives.

The last statement is a bit vague as long as one does not have a precise definition of a mixed motive, as was the case when Milne wrote his article. Since now we have at least triangulated categories of motives at our disposal, let me give a precise theorem.

Theorem 6.7.3. Suppose that the Tate-Beilinson conjecture 6.7.2 holds. Then, for any finite field $k$ :

(i) Voevodsky's triangulated category $D M_{\mathrm{gm}}(k, \mathbf{Q})$ is semi-simple in the sense that any exact triangle is a direct sum of split exact triangles.

(ii) The functor $\delta$ of (3.3.1) induces an equivalence of categories

$$
\begin{aligned}
\Delta: \operatorname{Mot}_{\text {num }}(k, \mathbf{Q})^{(\mathbf{Z})} & \stackrel{\sim}{\longrightarrow} D M_{\mathrm{gm}}(k, \mathbf{Q}) \\
\left(M_{i}\right) & \mapsto \bigoplus_{i \in \mathbf{Z}} \delta\left(M_{i}\right)[i] .
\end{aligned}
$$

(iii) Equivalently, (3.3.1) induces an equivalence of categories

$$
\Delta: D^{b}\left(\operatorname{Mot}_{\text {num }}(k, \mathbf{Q})\right) \stackrel{\sim}{\longrightarrow} D M_{\mathrm{gm}}(k, \mathbf{Q}) .
$$

Proof. First we check that, for $M, N \in \operatorname{Mot}_{\text {rat }}(k, \mathbf{Q})=\operatorname{Mot}_{\text {num }}(k, \mathbf{Q})$

$$
\operatorname{Hom}_{D M}(\delta(M), \delta(N)[i])= \begin{cases}0 & \text { for } i \neq 0 \\ \operatorname{Hom}_{M o t}(M, N) & \text { for } i=0\end{cases}
$$

For this, we reduce by duality to the case where $M=\mathbf{1}$, and then to the case where $N$ is of the form $h(X)(n)$ for $X$ smooth projective. Then the left hand side is $H^{i}(X, \mathbf{Q}(n))$ by $[198$, Cor. 3.2.7] and the cancellation theorem of Voevodsky [202], and the conclusion follows from Theorem 6.5.4.

This implies that $\Delta$ is fully faithful. To see that it is essentially surjective, using de Jong it now suffices to show that its essential image is thick, i.e. stable under exact triangles and direct summands. This follows from the following trivial but very useful lemma ( $c f$. [6, Lemma A.2.13]): in a semi-simple abelian category, any morphism is the direct sum of an isomorphism and a 0 morphism. The same lemma implies that $D M_{\mathrm{gm}}(k, \mathbf{Q})$ is semi-simple. Finally, (iii) is equivalent to (ii) because $\operatorname{Mot}_{\text {num }}(k, \mathbf{Q})$ is semi-simple.

Remarks 6.7.4. 1) This implies trivially a number of conjectures: the existence of a motivic $t$-structure on $D M_{\mathrm{gm}}(k, \mathbf{Q})$, the semi-simplicity of Galois action, independence of $l \ldots$

2) Theorem 6.7.3 (i) extends to $D M(k, \mathbf{Q})$, hence to $\mathcal{S H}(k, \mathbf{Q})$ (see 3.5). 
Next, the Tate-Beilinson conjecture implies the Lichtenbaum conjecture of the previous section. This was proven in [90] after localising at $l$ and under resolution of singularities, but using the higher Chow groups version of $\mathbf{Z}(n)$ we can get rid of the last assumption. Localising at $l$ can also be got rid of. In fact one can get a version of the Lichtenbaum conjecture for arbitrary, not just smooth projective, schemes of finite type over $\mathbf{F}_{p}$ : (ii) is especially well-adapted to this. We shall give details on this in Theorem 6.8.11. For the moment, let us sketch a proof of:

Theorem 6.7.5. The Tate-Beilinson conjecture 6.7.2 implies the Soulé conjecture 6.3.1.

Sketch. We shall derive the form of Remark 6.3.2 2). In view of (6.3.4), it is sufficient to prove the stronger equality

$$
\operatorname{ord}_{s=n} \operatorname{det}\left(1-F_{X} q^{-s} \mid H_{c}^{i}\left(\bar{X}, \mathbf{Q}_{l}\right)\right)=\operatorname{dim}_{\mathbf{Q}} H_{i}^{B M}(X, \mathbf{Q}(n))
$$

for all $X, i, n$. Now the morphism (6.7.1) has a homological version

$$
H_{i}^{B M, \text { ét }}\left(X, \mathbf{Z}(n) \otimes \mathbf{Z}_{l}(0)^{c}\right) \rightarrow H_{i}^{c, \text { cont }}\left(X, \mathbf{Z}_{l}(n)\right)
$$

where the left hand side is Borel-Moore étale motivic homology as explained in Subsection 4.4 (see the end for the coefficients $\left.\mathbf{Z}_{l}(0)^{c}\right)$ and the right hand side is the continuous version of Borel-Moore étale homology (relative to $\mathbf{F}_{p}$ ).

Lemma 6.7.6. Under the Tate-Beilinson conjecture 6.7.2, (6.7.4) is an isomorphism for any $X, i, n$.

This is easily proven by a dévissage using the localisation exact sequence plus Poincaré duality for both sides.

From this one deduces isomorphisms

$$
H_{i}^{B M}(X, \mathbf{Q}(n)) \otimes \mathbf{Q}_{l} \stackrel{\sim}{\longrightarrow} H_{i}^{c, \text { cont }}\left(\bar{X}, \mathbf{Q}_{l}(n)\right)^{G}
$$

with $G=G_{\mathbf{F}_{p}}$, and moreover that $G$ acts semi-simply on $H_{c, \text { cont }}^{i}\left(\bar{X}, \mathbf{Q}_{l}(n)\right)$ at the eigenvalue 1 (this means that the characteristic subspace corresponding to the eigenvalue 1 is semi-simple). Since $H_{c, \text { cont }}^{i}\left(\bar{X}, \mathbf{Q}_{l}(n)\right)$ is dual to $H_{i}^{c, \text { cont }}\left(\bar{X}, \mathbf{Q}_{l}(-n)\right)$, the result follows.

The first instance I know of this dévissage argument is [78, Th. 12.7]. Jannsen assumed resolution of singularities there but this is now unnecessary thanks to de Jong's theorem.

Since any finitely generated field $K$ over $\mathbf{F}_{p}$ is a filtering direct limit of finitely generated smooth $\mathbf{F}_{p}$-algebras and any smooth variety over $K$ is a filtering inverse limit of smooth varieties over $\mathbf{F}_{p}$, one also gets consequences of the Tate-Beilinson conjecture for such varieties. Typically: 
Theorem 6.7.7 ([90, Th. 8.32]). The Tate-Beilinson conjecture 6.7.2 implies the following for any finitely generated field $K / \mathbf{F}_{p}$ and any smooth projective variety $X / K$ :

(i) (Tate conjecture) The map $C H^{n}(X) \otimes \mathbf{Q}_{l} \rightarrow H_{\text {cont }}^{2 n}\left(\bar{X}, \mathbf{Q}_{l}(n)\right)^{G_{K}}$ is surjective, where $G_{K}=\operatorname{Gal}(\bar{K} / K)$.

(ii) The action of $G_{K}$ on $H_{\text {cont }}^{*}\left(\bar{X}, \mathbf{Q}_{l}\right)$ is semi-simple.

(iii) The cycle map $C H^{i}(X) \otimes \mathbf{Q}_{l} \rightarrow \tilde{H}_{\text {cont }}^{2 i}\left(X / \mathbf{F}_{p}, \mathbf{Q}_{l}(i)\right)$ is injective for all $i$.

In (iii), the group $\tilde{H}_{\text {cont }}^{2 i}\left(X / \mathbf{F}_{p}, \mathbf{Q}_{l}(i)\right)$ is by definition the direct limit of the $H_{\text {cont }}^{2 i}\left(\mathcal{X}, \mathbf{Q}_{l}(i)\right)$, where $\mathcal{X}$ runs through the smooth models of $X$ of finite type over $\mathbf{F}_{p}$.

Sketch. Extend $X$ to a smooth, projective morphism $f: \mathcal{X} \rightarrow U$ over a suitable smooth model $U$ of $K$. By Hard Lefschetz and Deligne's degeneration criterion [38], the Leray spectral sequence

$$
E_{2}^{p q}=H_{\text {cont }}^{p}\left(U, R^{q} f_{*}^{l-\text { adic }} \mathbf{Q}_{l}(n)\right) \Rightarrow H_{\text {cont }}^{p+q}\left(\mathcal{X}, \mathbf{Q}_{l}(n)\right)
$$

degenerates. (i) follows rather easily from this and the conjecture. The semisimplicity statement (ii) is only proven in [90] at the eigenvalue 1: the proof consists roughly of "hooking" the geometric semi-simplicity theorem of Deligne [34, Cor. 3.4.13] on the arithmetic semi-simplicity (special case $K=k)$. One can however prove it in general by using some folklore $([105,80]$ and the argument in [46, pp. 212-213] ${ }^{11}$ ), cf. [96]. The proof of (iii) is a simple direct limit argument.

Here are two other nice consequences (the proofs are the same as for [95, Cor. 2.6 and Th. 4.6], using Theorem 5.0.2 a)):

Theorem 6.7.8. Assume the Tate-Beilinson and the Bloch-Kato conjectures 6.7.2 and 4.2.3. Then

a) Gersten's conjecture for algebraic K-theory holds for any discrete valuation ring (hence for any local ring of a scheme smooth over a discrete valuation ring by Gillet-Levine [64]).

b) For any field $K$ of characteristic $p$ one has canonical isomorphisms

$$
K_{n}^{M}(K) \oplus \bigoplus_{0 \leq i \leq n-1} H^{2 i-n-1}\left(K,(\mathbf{Q} / \mathbf{Z})^{\prime}(i)\right) \stackrel{\sim}{\longrightarrow} K_{n}(K)
$$

where $(\mathbf{Q} / \mathbf{Z})^{\prime}(i)=\varliminf_{(m, \operatorname{char} k)=1} \mu_{m}^{\otimes i}$. The spectral sequence $(2.2 .3)$ canonically degenerates.

\footnotetext{
${ }^{11}$ Which I am grateful to Yves André for explaining me.
} 
By [191, Prop. 2.6 and Th. 3.1]), Theorem 6.7 .7 (i) and (ii) imply the standard conjecture HN on page 17 (hence the other ones: B and C). On the other hand, by an argument similar to that in [81, Lemma 2.7], (iii) implies the filtration conjecture of Bloch-Beilinson-Murre. As was proved by Peter O'Sullivan, the latter implies Voevodsky's conjecture 3.1.2 (see [3, Th. 10.5.2.3]). Also, under the Bloch-Beilinson-Murre conjecture, Hanamura's vanishing conjecture (Van) in [71, III] may be reformulated as follows: for any smooth projective $X / K$, one has

$$
H^{q}\left(h^{\geq i} X, \mathbf{Q}(n)\right)=0 \text { for } \begin{cases}q \leq i & \text { if } q \neq 2 n \\ q<i & \text { if } q=2 n\end{cases}
$$

where $h^{\geq i}(X)$ denotes the part of weight $\geq i$ of $h(X) \in \operatorname{Mot}_{\text {rat }}(K, \mathbf{Q})$ under the Bloch-Beilinson-Murre filtration. It would be sufficient to have this vanishing in order to get a motivic $t$-structure on his category, but I have not derived it from the Tate-Beilinson conjecture. Presumably one should first prove a version of (Van) relative to a smooth model of $K$ and then pass to the limit as we did for the Beilinson-Soulé conjecture: this looks feasible but fairly technical.

Another conjecture I don't know how to derive from the Tate-Beilinson conjecture is the Hodge index standard conjecture, see [109, §5].

\subsection{Lichtenbaum's Weil-étale topology; reformulation of his second conjecture}

In [126], Lichtenbaum introduced a new Grothendieck topology on schemes of characteristic $p$ : he christened it Weil-étale topology. This leads to a fundamental clarification of the formulation of his previous conjectures, and of what should be true or not in terms of finite generation conjectures.

Roughly, Lichtenbaum replaces the Galois group $\operatorname{Gal}\left(\overline{\mathbf{F}}_{q} / \mathbf{F}_{q}\right) \simeq \hat{\mathbf{Z}}$ by its dense subgroup generated by Frobenius $(\simeq \mathbf{Z}$ ) and extends this idea (which of course goes back to Weil) to schemes of higher dimension. The corresponding cohomology theory should be called Weil-étale cohomology. I find this terminology awkward because it can create confusion with a "Weil cohomology", especially as most known Weil cohomology theories in characteristic $p$ are based on étale cohomology! For this reason, and also as a tribute to Lichtenbaum's paternity, I prefer to rechristen it Lichtenbaum cohomology, while keeping his notation $H_{W}^{*}(X, \mathcal{F})$ which recalls Weil's contribution.

We may take the hypercohomology of Bloch's cycle complexes (or the Suslin-Voevodsky complexes) in the Weil-étale topology and get Lichtenbaum motivic cohomology ${ }^{12} H_{W}^{i}(X, \mathbf{Z}(n))$. The various motivic cohomology groups map to each other as follows:

\footnotetext{
${ }^{12}$ Thereby conflicting with a terminology briefly introduced by Voevodsky for étale
} motivic cohomology... 


$$
H^{i}(X, \mathbf{Z}(n)) \rightarrow H_{\text {ét }}^{i}(X, \mathbf{Z}(n)) \rightarrow H_{W}^{i}(X, \mathbf{Z}(n)) .
$$

Lichtenbaum's cohomology has been developed by Geisser in [58]. His main results are the following:

Theorem 6.8.1 (Geisser). Let $\varepsilon$ be the projection of the Weil-étale site onto the (usual) étale site. Then for any complex of étale sheaves $C$,

(i) There is a quasi-isomorphism

$$
R \varepsilon_{*} \varepsilon^{*} C \simeq C \stackrel{L}{\otimes} R \varepsilon_{*} \varepsilon^{*} \mathbf{Z} .
$$

(ii) There are long exact sequences

$$
\begin{aligned}
\cdots \rightarrow H_{\text {êt }}^{i}(X, C) \rightarrow & H_{W}^{i}\left(X, \varepsilon^{*} C\right) \\
& \rightarrow H_{\text {êt }}^{i-1}(X, C) \otimes \mathbf{Q} \stackrel{\partial}{\rightarrow} H_{\text {ét }}^{i+1}(X, C) \rightarrow \ldots
\end{aligned}
$$

Moreover $R \varepsilon_{*} \varepsilon^{*} \mathbf{Z} \simeq \mathbf{Z}^{c}$, where $\mathbf{Z}^{c}$ is the complex alluded to in (6.7.3).

As important special cases, which give a feel of Lichtenbaum cohomology, we get:

Corollary 6.8.2. (i) $H_{\text {êt }}^{i}(X, C) \stackrel{\sim}{\longrightarrow} H_{W}^{i}\left(X, \varepsilon^{*} C\right)$ if the cohomology sheaves of $C$ are torsion.

(ii) $H_{W}^{i}\left(X, \varepsilon^{*} C\right) \simeq H_{\text {êt }}^{i}(X, C) \oplus H_{\text {êt }}^{i-1}(X, C)$ if the cohomology sheaves of $C$ are $\mathbf{Q}$-vector spaces.

In the isomorphism of (ii), a very important element shows up: the generator $e$ of $H_{W}^{1}\left(\mathbf{F}_{p}, \mathbf{Z}\right) \simeq \mathbf{Z}$ (normalised, say, by sending the geometric Frobenius to 1 ).

The sequence (6.8.1) is completely similar to one derived from the TateBeilinson conjecture in [90, Prop. 9.12] - except that it is not conjectural. With this and the last result of Theorem 6.8.1, everything falls into place and we are able to give a much more understandable reformulation of Conjecture (ii) in Theorem 6.7.1:

Theorem 6.8.3. The Tate-Beilinson conjecture is also equivalent to the following one: the map

$$
(\varepsilon \alpha)^{*} \mathbf{Z}(n) \otimes \mathbf{Z}_{l} \rightarrow \varepsilon^{*} \mathbf{Z}_{l}(n)^{c}
$$

induces isomorphisms on Weil-étale cohomology groups

$$
H_{W}^{*}(X, \mathbf{Z}(n)) \otimes \mathbf{Z}_{l} \stackrel{\sim}{\longrightarrow} H_{\text {cont }}^{*}\left(X, \mathbf{Z}_{l}(n)\right)
$$

for all smooth $X$ if $l \neq p$ (resp. for all smooth projective $X$ if $l=p$ ).

Geisser's theorem also allows us to reformulate Lichtenbaum's conjectures in terms of Lichtenbaum motivic cohomology (cf. [95, Cor. 3.8]): 
Conjecture 6.8.4. Let $X$ be a smooth projective variety over $\mathbf{F}_{p}$, and $d=$ $\operatorname{dim} X$.

a) The pairing

$$
H_{W}^{2 n}(X, \mathbf{Z}(n)) \times H_{W}^{2 d-2 n}(X, \mathbf{Z}(d-n)) \rightarrow H_{W}^{2 d}(X, \mathbf{Z}(d)) \rightarrow \mathbf{Z}
$$

is nondegenerate modulo torsion for all $n$.

b) For any $(i, n)$, the pairing

$$
\begin{aligned}
H_{W}^{i}(X, \mathbf{Z}(n))_{\text {tors }} \times H_{W}^{2 d+1-i}(X, \mathbf{Q} / \mathbf{Z}(d-n)) & \\
& \rightarrow H_{W}^{2 d+1}(X, \mathbf{Q} / \mathbf{Z}(d)) \stackrel{\sim}{\longrightarrow} \mathbf{Q} / \mathbf{Z}
\end{aligned}
$$

induces a perfect pairing of finite groups

$$
H_{W}^{i}(X, \mathbf{Z}(n))_{\text {tors }} \times H_{W}^{2 d+2-i}(X, \mathbf{Z}(d-n))_{\text {tors }} \rightarrow \mathbf{Q} / \mathbf{Z} .
$$

c) $H_{W}^{i}(X, \mathbf{Z}(n))$ is finitely generated, finite for $i \notin\{2 n, 2 n+1\}$ and 0 for $i \leq 0$ (if $n>0$ ).

d) The kernel and cokernel of cup-product by $e$ (generator of $H_{W}^{1}\left(\mathbf{F}_{p}, \mathbf{Z}\right)$ )

$$
H_{W}^{2 n}(X, \mathbf{Z}(n)) \rightarrow H_{W}^{2 n+1}(X, \mathbf{Z}(n))
$$

are finite.

e) The canonical homomorphism

$$
H_{\text {ét }}^{i}(X, \mathbf{Z}(n)) \rightarrow H_{W}^{i}(X, \mathbf{Z}(n))
$$

is an isomorphism for $i \leq 2 n$.

Concerning the zeta function $\zeta(X, s)$, the following much nicer reformulation is due to Geisser (op. cit.):

\section{Conjecture 6.8.5.}

1. $\operatorname{ord}_{s=n} \zeta(X, s)=-\operatorname{rk} C H^{n}(X):=-m(n)$.

2. $\lim _{s \rightarrow n}\left(1-q^{n-s}\right)^{m(n)} \zeta(X, s)= \pm q^{\chi\left(X, \mathcal{O}_{X}, n\right)} \chi(X, \mathbf{Z}(n))$, where

$$
\begin{gathered}
\chi(X, \mathbf{Z}(n))=\prod_{i}\left|H_{W}^{i}(X, \mathbf{Z}(n))_{\text {tors }}\right|^{(-1)^{i}} \cdot R_{n}(X)^{-1}, \\
\chi\left(X, \mathcal{O}_{X}, n\right)=\sum_{\substack{0 \leq i \leq n \\
0 \leq j \leq d}}(-1)^{i+j}(n-i) h_{i j}, \quad h_{i j}=\operatorname{dim} H^{j}\left(X, \Omega^{i}\right)
\end{gathered}
$$

and $R_{n}(X)$ is the absolute value of the determinant of the pairing (6.8.3) (modulo torsion) with respect to arbitrary bases of $H_{W}^{2 n}(X, \mathbf{Z}(n)) /$ tors and $H_{W}^{2 d-2 n}(X, \mathbf{Z}(d-n)) /$ tors. 
Here I would like to correct a mistake in [95, Remark 3.11] about the sign. It is stated there that this sign is $(-1)^{\sum_{a>n} m(a)}$. However, the Weil conjecture only says that the real zeroes of $\zeta(X, s)$ are half integers, so the correct formula is $(-1)^{\sum_{a / 2>n} m(a / 2)}$, where $a$ is an integer. By semi-simplicity, the value of $m(a / 2)$ is the multiplicity of the eigenvalue $q^{a / 2}$ (positive square root) for the action of Frobenius on $H^{a}\left(\bar{X}, \mathbf{Q}_{l}\right)$. This multiplicity may well be nonzero, for example if $X$ is a supersingular elliptic curve: I am grateful to A. Chambert-Loir for raising this issue. I have no idea how to relate $m(a / 2)$ to cycle-theoretic invariants: there are no half Tate twists or half-dimensional Chow groups...

Theorem 6.8.6. The Tate-Beilinson conjecture 6.7.2 implies Conjectures 6.8.4 and 6.8.5 (hence Lichtenbaum's conjectures in Subsection 6.6).

Sketch. (For details, see [95].) By the finiteness results on étale cohomology, the right hand side, hence the left hand side of (6.8.2) is a finitely generated $\mathbf{Z}_{l^{-}}$ module. Hence, by faithful flatness, $H_{W}^{*}(X, \mathbf{Z}(n)) \otimes \mathbf{Z}_{(l)}$ is a finitely generated $\mathbf{Z}_{(l)}$-module. From there it is tempting to descend directly to $\mathbf{Z}$, but this is wrong as Lichtenbaum pointed out several years ago: for example, the $\mathbf{Z}$ module $M=\bigoplus \mathbf{Z} / l$ is such that $M \otimes \mathbf{z} \mathbf{Z}_{(l)}$ is finitely generated over $\mathbf{Z}_{(l)}$ for all $l$, while it is certainly not finitely generated. For a torsion-free example, take the subgroup of $\mathbf{Q}$ formed of all fractions with square-free denominator. ${ }^{13}$ A correct proof uses a duality argument, which is encapslated in Lemma 6.8.7 below. (Arithmetic) Poincaré duality for continuous étale cohomology allows us to apply this duality argument. This basically explains the proof of a), b), c) and d); as for e), it follows from (6.8.1) and the Beilinson-Parshin conjecture 6.5.3. Finally, the deduction of Conjecture 6.8.5 is not especially new and goes back to Milne [137, Th. 4.3 and Cor. 5.5] (see also [90, Cor. 7.10 and Th. 9.20] and [58, Proof of Th. 8.1]).

Lemma 6.8.7 ([95, Lemma 3.9]). Let $R$ be a commutative ring and $A \times$ $B \rightarrow R$ a pairing of two flat $R$-modules $A, B$.

a) Suppose that this pairing becomes non-degenerate after tensoring by $R_{l}$ for some prime ideal $l$ of $R$, where $R_{l}$ denotes the completion of $R$ at $l$. Then it is non-degenerate.

b) Suppose that $R$ is a noetherian domain and let $K$ be its field of fractions. If, moreover, $\operatorname{dim}_{K} A \otimes K<\infty$ or $\operatorname{dim}_{K} B \otimes K<\infty$, then $A$ and $B$ are finitely generated.

${ }^{13}$ One should be careful that this mistake can be found in the literature, e.g. see in [135, Proof of Th. 12.5] the proof that the group of morphisms between two abelian varieties is finitely generated; the corresponding proof in [147, p. 177] is completely correct. Lemma 6.8 .7 will also justify the proof of $[134$, Ch. VI, Th. 11.7]. 
Corollary 6.8.8. Under the Tate-Beilinson conjecture 6.7.2:

a) $\operatorname{dim}_{\mathbf{Q}} H^{i}(X, \mathbf{Q}(n))<\infty$ for all $i, n$.

b) Assuming further the Beilinson-Lichtenbaum Conjecture 4.2.4, $H^{i}(X, \mathbf{Z}(n))$ is finitely generated for any $i \leq n+2$.

Corollary 6.8.9. Under the Tate-Beilinson conjecture 6.7.2 and the BlochKato conjecture 4.2.3, the Bass and motivic Bass conjectures 6.1.1 and 6.1.2 are true in the following cases for smooth projective varieties $X$ over $\mathbf{F}_{p}$ :

(i) $d=\operatorname{dim} X \leq 3$.

(ii) (for Conjecture 6.1.2 b):) $n \leq 2$.

Proof. (i) It suffices to prove Conjecture 6.1.2 (for Conjecture 6.1.1, use the spectral sequence (2.2.3)). Independently of any conjecture one has $H^{i}(X, \mathbf{Z}(n))=0$ for $i>n+d$ (in fact, the group of cycles $z^{n}(X, 2 n-i)$ itself is 0$)$. For $i=n+d$, by the coniveau spectral sequence for motivic cohomology, this a group is a quotient of

$$
\bigoplus_{x \in X_{(0)}} H^{n-d}(k(x), \mathbf{Z}(n-d)) .
$$

The latter group is 0 for $n<d$ and also for $n \geq d+2$ by Theorem 2.2.1, since Milnor's $K$-groups of finite fields vanish in degree $\geq 2$. So far we have only used that $X$ is smooth. Suppose now $X$ smooth projective: for $n=d$, $H^{n+d}(X, \mathbf{Z}(n))=C H_{0}(X)$ is finitely generated by Bloch [18] (see also KatoSaito [102]), and for $n=d+1$ it is isomorphic to $k^{*}$ by Akhtar [7]. If $d \leq 3$, this plus Corollary 6.8.8 b) covers all motivic cohomology.

(ii) Same argument, noting that for $n \leq 2$ Corollary 6.8.8 b) again covers all the motivic cohomology of $X$.

Remarks 6.8.10. 1) Trying to extend Corollary 6.8.9 to open varieties via de Jong's theorem is a little delicate: we can apply part 2 of the argument in the proof of Lemma 2.4.3 provided we have an a priori control of the torsion of the motivic cohomology groups involved. By the Beilinson-Lichtenbaum conjecture, the group $H^{i}(X, \mathbf{Z} / m(n))$ is finite for any smooth variety $X$ as long as $i \leq n+1$. This implies that ${ }_{m} H^{i}(X, \mathbf{Z}(n))$ is finite as long as $i \leq n+2$, so that Corollary 6.8.9 goes through for arbitrary smooth varieties as long as $d \leq 2$ or $n \leq 2$, because then this finiteness covers all motivic cohomology groups. For $d=3$ we have a problem with $H^{6}(X, \mathbf{Z}(3))$ and $H^{7}(X, \mathbf{Z}(4))$, however. Unfortunately, Abyankhar's resolution of singularities for 3 -folds in characteristic $>5[1$, Th. (13.1)] only works over an algebraically closed field.

2) For singular schemes $X$, we may introduce Lichtenbaum Borel-Moore motivic homology groups as in Subsection 4.4, using the Lichtenbaum topology rather than the étale topology, or define them as

$$
H_{i}^{B M, W}(X, \mathbf{Z}[1 / p](n))=H_{i}^{B M, \text { ét }}\left(X, \mathbf{Z}[1 / p](n) \stackrel{L}{\otimes} \mathbf{Z}^{c}\right)
$$


cf. (6.7.3) and (6.7.4). Then, under the Tate-Beilinson conjecture, the groups $H_{i}^{W, B M}(X, \mathbf{Z}[1 / p](n))$ are all finitely generated $\mathbf{Z}[1 / p]$-modules. This follows by dévissage from the smooth projective case.

3) Corollary 6.8.9 applies trivially when $X$ is a curve. Hence we get (under the Bloch-Kato conjecture) that all the motivic cohomology of $X$ is finitely generated, by a method totally different from that in the proof of Theorem 6.1.5! Using the spectral sequence (2.2.3) we can then recover Quillen's finite generation theorem for algebraic $K$-theory...

Finally, let us give a version of Conjecture 6.8 .5 for an arbitrary $\mathbf{F}_{p^{-}}$scheme of finite type, and explain that it follows from the Tate-Beilinson conjecture. It rests on Remark 6.8.10 2).

Theorem 6.8.11. Let $X$ be a scheme of finite type over $\mathbf{F}_{p}$. If the TateBeilinson conjecture 6.7.2 holds, then, for any $n \in \mathbf{Z}$ :

(i) $\operatorname{ord}_{s=n} \zeta(X, s)=\sum_{i \in \mathbf{Z}}(-1)^{i} i \operatorname{rk} H_{i}^{W, B M}(X, \mathbf{Z}[1 / p](n)):=-m(n)$.

(ii) The cohomology groups of the complex

$$
\cdots \rightarrow H_{i}^{W, B M}(X, \mathbf{Z}[1 / p](n)) \stackrel{\cdot e}{\longrightarrow} H_{i-1}^{W, B M}(X, \mathbf{Z}[1 / p](n)) \rightarrow \ldots
$$

are finite, where $e$ is the canonical generator of $H_{W}^{1}\left(\mathbf{F}_{p}, \mathbf{Z}\right) \simeq \mathbf{Z}$.

(iii) Up to \pm a power of $p$, one has

$$
\lim _{s \rightarrow n}\left(1-q^{n-s}\right)^{m(n)} \zeta(X, s)=\chi\left(H_{*}^{W, B M}(X, \mathbf{Z}[1 / p](n)), \cdot e\right) .
$$

Sketch. A version of this for $l$-adic cohomology with compact supports was proven in [90, Th. 7.8] under the assumption that Galois acts semi-simply at the eigenvalue 1 ( $c f$. Remark 6.7.4 1). One passes from there to Borel-Moore $l$-adic homology by arithmetic duality (cf. loc. cit., Th. 3.17). It is actually simpler to redo the proof of [90, Th. 7.8] with Borel-Moore $l$-adic homology by using a description of $\zeta(X, s)$ in these terms, which only involves duality for the geometric groups with $\mathbf{Q}_{l}$ coefficients. One then concludes thanks to Lemma 6.7.6.

Remarks 6.8.12. 1) This approach does not handle the missing power of $p$. This has recently been achieved by Geisser [60]: his point of view is to define a compactly supported version of Lichtenbaum's cohomology. To get the right groups he refines Lichtenbaum's topology by adding cdh coverings to it, which unfortunately forces him to assume resolution of singularities. Presumably, the corresponding non-compactly supported cohomology (for smooth schemes) involves the logarithmic part of Mokrane's de Rham-Witt cohomology with logarithmic poles at infinity [141] (whose definition unfortunately also assumes resolution of singularities), glued to motivic cohomology in a similar way as (4.4.2). Can one give a direct definition of this motivic cohomology?

2) In characteristic 0, Lichtenbaum has an exactly parallel formulation of an integral conjecture for the special values of the zeta function, in terms of his cohomology still under development. 


\section{Unconditional results: varieties of abelian type over finite fields}

\subsection{Main result}

We shall give cases in which we can prove the Tate-Beilinson conjecture 6.7.2. Namely, let $\mathcal{A}=\operatorname{Mot}_{\text {rat }}(k, \mathbf{Q})$.

Definition 7.1.1. a) Let $\mathcal{A}_{\mathrm{ab}}$ be the thick rigid subcategory of $\mathcal{A}$ generated by Artin motives and motives of abelian varieties.

b) $B(k)=\left\{X \mid h(X) \in \mathcal{A}_{\mathrm{ab}}\right\}$.

c) $B_{\text {tate }}(k)=\{X \in B(k) \mid$ the Tate conjecture holds for the $l$-adic cohomology of $X$ for some $l \neq \operatorname{char} k\}$.

(In c), this does not depend on $l$ because Frobenius acts semi-simply on $\left.H^{*}\left(\bar{X}, \mathbf{Q}_{l}\right).\right)$

Examples 7.1.2. 1) $X \in B(k)$ and $\operatorname{dim} X \leq 3 \Rightarrow X \in B_{\text {tate }}(k)$. This is a slight strengthening of Soulé [175, Th. 4 i)]: the problem is that Soulé works with a collection $A(k)$ of varieties such that, clearly, $A(k) \subseteq B(k)$, but I don't know if equality holds, so that this claim unfortunately does not follow from [175], contrary to what was indicated in [95, Example $1 \mathrm{~b}$ )]. For this reason I shall justify it in Subsection 7.5.

2) Products of elliptic curves are in $B_{\text {tate }}(k)$ (Spieß [178]).

3) There are many examples of abelian varieties in $B_{\text {tate }}(k)$ (Zarhin, Lenstra, Milne): powers of simple abelian varieties "of $K 3$ type" or "of ordinary type", etc. $[212,112,139]$.

4) Certain Fermat hypersurfaces (Tate, Katsura-Shioda [188, 103]).

The main result of [95] is:

Theorem 7.1.3. Conjectures 6.5.1 and 6.5.2 are true for $X \in B_{\text {tate }}(k)$.

Of course, it is not difficult to get Conjecture 6.5.1: indeed, Galois action on the $l$-adic cohomology of $X$ is semi-simple (reduce to an abelian variety $A$ and use the fact that the arithmetic Frobenius is the inverse of the geometric Frobenius, which is central in the semi-simple algebra $\operatorname{End}(A) \otimes \mathbf{Q})$. By [191], this plus the cohomological Tate conjecture imply Conjecture 6.5.1. What is new is to obtain Conjecture 6.5.2. We shall explain in the sequel of this section how this follows from the Kimura-O'Sullivan theory of "finite dimensional" Chow motives.

In the previous sections, we referred to [95] for proofs or details of proofs on some consequences of the Tate-Beilinson conjecture. In loc. cit. , the corresponding proofs are given for varieties in $B_{\text {tate }}(k)$, and yield unconditional theorems. 


\subsection{The Soulé-Geisser argument}

This argument is first found in Soulé's paper [175] and was amplified by Geisser in $[56]^{14}$. It is really a weight argument and is very simple to explain: suppose that Frobenius acts on some group $H$ and that

- For one reason we know that it acts by multiplication by some power of $p$, say $p^{n}$.

- For another reason we know that it is killed by some polynomial $P$ with integral coefficients.

If we can prove that $P\left(p^{n}\right) \neq 0$, then we get that $H \otimes \mathbf{Q}=0$ (more precisely, that $H$ is torsion of exponent dividing $\left.P\left(p^{n}\right)\right)$.

Typically, $H$ will be a Hom group between a certain motive $M$ and a Tate motive ( $M$ might also be a shift of a pure motive in $\left.D M_{\mathrm{gm}}(k)\right)$. The issue is then to show that the characteristic polynomial of the Frobenius endomorphism of $M$, assuming that this polynomial exists, is not divisible by $T-p^{n}$. A nilpotence theorem will allow us to prove this below.

\subsection{The Kimura-O'Sullivan theory}

This theory was developed independently by S. I. Kimura [106] and P. O'Sullivan [150]. An abstract version (which is also most of O'Sullivan's point of view) is developed in [6, §9]. See also André's recent Bourbaki talk [4].

Definition 7.3.1. Let $\mathcal{A}$ be a $\mathrm{Q}$-linear tensor category. An object $M \in \mathcal{A}$ is even if some exterior power of $M$ vanishes, odd if some symmetric power of $M$ vanishes, finite dimensional if it is a direct sum of a even and an odd object.

(Kimura says evenly and oddly finite dimensional; O'Sullivan says positive and negative, and semi-positive instead of finite-dimensional.)

There are two reasons why finite dimensionality is an important notion: first its remarkable stability properties, and second Kimura's nilpotence theorem.

Theorem 7.3.2 (Kimura [106, Cor. 5.11, Prop. 6.9], O'Sullivan). Suppose $\mathcal{A}$ rigid. Then the full subcategory $\mathcal{A}_{\text {kim }}$ of $\mathcal{A}$ formed of finite dimensional objects is thick and rigid, i.e. stable under direct sums, direct summands, tensor products and duals.

Kimura developed his theory for $\mathcal{A}=\operatorname{Mot}_{\text {rat }}(K, \mathbf{Q})(K$ a field $)$ and proved a nilpotence theorem for correspondences on a finite dimensional motive which are homologically equivalent to 0 . This theorem was slightly strengthened in

$\overline{14}$ The reader should also look at Coombes' paper [33] where the author uses Soulé's work to get a $K$-cohomological variant of Lichtenbaum's conjecture for the zeta function of a rational surface over a finite field: I am grateful to the referee for pointing out this paper. 
[6], replacing homological by numerical equivalence (and nil by nilpotent). See [6, Prop. 9.1.14] for an abstract statement. In the case of Chow motives, this gives:

Theorem 7.3.3. Let $M \in \mathcal{A}=\operatorname{Mot}_{\text {rat }}(K, \mathbf{Q})$ and $\bar{M}$ its image in $\overline{\mathcal{A}}=$ $\operatorname{Mot}_{\text {num }}(k, \mathbf{Q})$. Then the kernel of $\mathcal{A}(M, M) \rightarrow \overline{\mathcal{A}}(\bar{M}, \bar{M})$ is a nilpotent ideal.

All this theory would be nice but rather formal if one had no examples of finite dimensional motives. Fortunately, there are quite a few:

Theorem 7.3.4 (Kimura $[106$, Th. 4.2], O'Sullivan). For $\mathcal{A}=$ $\operatorname{Mot}_{\text {rat }}(K, \mathbf{Q}), \mathcal{A}_{\text {kim }} \supset \mathcal{A}_{\mathrm{ab}}$.

The proof is essentially a reformulation of Šermenev's proof of the Künneth decomposition of the Chow motive of an abelian variety [180].

Kimura-O'Sullivan conjecture 7.3.5. Let $K$ be a field and $\mathcal{A}=$ $\operatorname{Mot}_{\text {rat }}(K, \mathbf{Q})$. Then $\mathcal{A}=\mathcal{A}_{\text {kim }}$.

By [6, Ex. 9.2.4], this conjecture follows from the standard conjecture on Künneth projectors and the existence of the Bloch-Beilinson-Murre filtration.

\subsection{The proof}

The proof of Conjecture 6.5.2 in Theorem 7.1.3 is fairly simple: the nilpotence theorem 7.3.3 is used three times. First, decompose the numerical motive $\bar{h}(X)$ into a direct sum of simple motives by Jannsen's Theorem 3.1.1. By nilpotence, this decomposition lifts to Chow motives, hence we may replace $h(X)$ by a Chow motive $S$ whose numerical image $\bar{S}$ is simple. We need to show that

$$
\mathcal{A}\left(S, L^{n}\right) \stackrel{\sim}{\longrightarrow} \overline{\mathcal{A}}\left(\bar{S}, \bar{L}^{n}\right)
$$

for any $n$, where $L$ is the Lefschetz motive. Then we have the usual dichotomy:

a) $\bar{S} \simeq \bar{L}^{n}$. Then, by nilpotence, $S \simeq L^{n}$ and this is obvious.

b) $\bar{S} \not \bar{L}^{n}$. Then the right hand side of (7.4.1) is 0 and we have to show that the left hand side is also 0. By [138, Prop. 2.6], the characteristic polynomial $P$ of the Frobenius endomorphism $F_{\bar{S}}$ of $\bar{S}$ is not $T-q^{n}$. But, by nilpotence, there is an $N>0$ such that $P\left(F_{S}\right)^{N}=0$. The conclusion now follows by the Soulé-Geisser argument.

\subsection{Justification of Example 7.1.2 1)}

We shall actually prove directly:

Theorem 7.5.1. If $X \in B(k)$ and $d=\operatorname{dim} X \leq 3$, then the Tate-Beilinson conjecture holds for $X$. 
Proof. In general, let $M \in \mathcal{A}_{\text {kim }}$ and $\bar{M}$ be its image in $\bar{A}$. By Theorem 7.3.3, the weight grading $\bar{M}=\bigoplus \bar{M}^{(i)}$ ( $c f$. p. 18) lifts to a grading $M=\bigoplus M^{(i)} .^{15}$ For simplicity, we shall say that an object $M \in \mathcal{A}$ is of weight $i$ if $\bar{M}$ is of weight $i$, so that $M^{(i)}$ is of weight $i$. Also, if $X$ is smooth projective and $h(X) \in \mathcal{A}_{\text {kim }}$, we simply write $h^{i}(X)$ for $h(X)^{(i)}$.

Let $M$ be of weight $2 n$; consider the following property:

(*) The natural homomorphism

$$
\mathcal{A}\left(M, L^{2 n}\right) \otimes \mathbf{Q}_{l} \rightarrow\left(H_{l}(M)(n)\right)^{G}
$$

is an isomorphism.

We need a lemma:

Lemma 7.5.2. Let $\mathcal{A}_{\mathrm{ab}}^{(2)}$ be the full subcategory of $\mathcal{A}_{\mathrm{ab}}$ formed of motives of weight 2. Then

a) Property $\left(^{*}\right)$ holds for all objects of $\mathcal{A}_{\mathrm{ab}}^{(2)}$.

b) If $M \in \mathcal{A}_{\mathrm{ab}}^{(2)}$, then $M^{\vee}(1) \in \mathcal{A}_{\mathrm{ab}}^{(2)}$.

Proof. a) We immediately reduce to the case where $M$ is of the form $h^{2}\left(A \otimes_{k} L\right)$ for $A$ an abelian variety and $L$ a finite extension of $k$; then it follows from Tate's theorem [189] and the finiteness of $\operatorname{Pic}^{0}(A)=A^{\vee}(k)$.

b) We reduce to the same case as in a). Thanks to Lieberman [127], any polarisation of $A$ induces via Poincaré duality an isomorphism $\bar{h}^{2}\left(A \otimes_{k} L\right)^{\vee} \simeq$ $\bar{h}^{2}\left(A \otimes_{k} L\right)(-1)$; by Theorem 7.3.3 this lifts to an isomorphism $h^{2}\left(A \otimes_{k} L\right)^{\vee} \simeq$ $h^{2}\left(A \otimes_{k} L\right)(-1)$.

Let now $X$ be as in Theorem 7.5.1. We must prove that, for all $n, h^{2 n}(X)$ verifies $\left(^{*}\right)$. For $n=0$ it is trivial. For $n=1$, it follows from Lemma 7.5.2 a). For $n=d-1$, it follows from Poincaré duality (lifted to $\mathcal{A}$ by Theorem 7.3.3) and Lemma 7.5.2 b). For $n=d$, it also follows by Poincaré duality from the case $d=0$ by the same argument as in the proof of Lemma 7.5.2 b). If $d \leq 3$, this covers all values of $n$.

Corollary 7.5.3. If $X \in B(k)$ and $\operatorname{dim} X \leq 3$, under the Bloch-Kato conjecture 4.2.3 all motivic cohomology groups of $X$ are finitely generated.

This follows from Theorem 7.5.1 and Corollary 6.8.9 (i).

Definition 7.5.4. A finitely generated field $K / \mathbf{F}_{p}$ is of abelian type if it is the function field of a smooth projective variety of abelian type.

$\overline{15}$ This grading is not necessarily unique, but the idempotents defining it are unique up to conjugation, hence the $M^{(i)}$ are unique up to isomorphism. 
Corollary 7.5.5. Let $X$ be an $\mathbf{F}_{p}$-scheme of finite type. Assume that $\operatorname{dim} X \leq$ 2 and that the function fields of all its irreducible components of dimension 2 are of abelian type. Then the conclusions of Theorem 6.8.11 hold for X.

Proof. This is just an effective case of Theorem 6.8.11. The point is that in the dévissage, the closed subvarieties one encounters are all of dimension $\leq 1$ and all smooth projective curves are of abelian type.

\section{Questions and speculations}

\subsection{The finite generation issue}

Recall that, by Theorem 6.8.6, the Tate-Beilinson conjecture implies the finite generation of the Lichtenbaum cohomology groups $H_{W}^{i}(X, \mathbf{Z}(n))$ for any smooth projective variety $X / \mathbf{F}_{p}$ and any $i, n$, and that by Remark 6.8.10 $2)$ this in turn implies the finite generation of $H_{i}^{W, B M}(X, \mathbf{Z}(n)) \otimes \mathbf{Z}[1 / p]$ over $\mathbf{Z}[1 / p]$ for any scheme $X$ of finite type over $\mathbf{F}_{p}$. In particular, $H_{*}^{W, B M}(X, \mathbf{Q}(n))$ is a finite-dimensional $\mathbf{Q}$-vector space which implies by an analogue of Corollary 6.8.2 (ii) (or by dévissage from the smooth case) the same result for usual Borel-Moore motivic homology $H_{*}^{B M}(X, \mathbf{Q}(n))$.

On the other hand, Corollary 6.8.9 and Remark 6.8.10 1) show that under the Beilinson-Lichtenbaum and the Tate-Beilinson conjecture, $H^{i}(X, \mathbf{Z}(n))$ is finitely generated for $X$ smooth in a certain range. The first case not reached is $C H^{3}(X)$ for $X$ a smooth projective 4-fold. It is explained in [95, Remark 4.10] that, under the two conjectures, the following conditions are equivalent:

1. $C H^{3}(X)$ is finitely generated.

2. $C H^{3}(X)_{\text {tors }}$ is finite.

3. $H_{\text {Zar }}^{0}\left(X, \mathcal{H}_{\text {ét }}^{4}\left((\mathbf{Q} / \mathbf{Z})^{\prime}(3)\right)\right.$ is finite (it is a priori of finite exponent).

I don't see any argument allowing one to deduce finite generation in this case from known conjectures. The only one I can think of is Kato's conjecture:

Kato conjecture 8.1.1 ([101, Conj. (0.3)]). For any smooth projective variety $X$ of dimension $d$ over $\mathbf{F}_{p}$ and any $m \geq 1$, the homology in degree $i$ of the Gersten complex

$$
0 \rightarrow \bigoplus_{x \in X_{(d)}} H_{\text {ét }}^{d+1}(k(x), \mathbf{Z} / m(d)) \rightarrow \cdots \rightarrow \bigoplus_{x \in X_{(0)}} H_{\text {ét }}^{1}(k(x), \mathbf{Z} / m) \rightarrow 0
$$

is $\left\{\begin{array}{ll}0 & \text { if } i>0 \\ \mathbf{Z} / m & \text { if } i=0\end{array}\right.$, the last isomorphism being induced by the trace map.

This conjecture is class field theory for $d=1$; it has been proven by Kato for $d=2$ [101], by Colliot-Thélène for general $d$ and $i \geq d-3$ if $m$ is prime to $p$ [31] and by Suwa under the same condition if $m$ is a power of $p$ [187]. 
However it does not seem to bear on the issue, except in limit cases (see below). I am therefore tempted to think that there is a counterexample to Conjecture 6.1.2. It might involve infinite 2-torsion; however if it involves infinite $l$-torsion for some odd prime $l$, it will yield an example where $K_{0}(X)$ is not finitely generated, disproving the original Bass conjecture (since the natural map $C H^{3}(X) \rightarrow \mathrm{gr}^{3} K_{0}(X)$ has kernel killed by $\left.(3-1) !=2\right)$.

This also sheds some doubt in my mind on [145, Conj. 1.1.4] (a homotopytheoretic Bass conjecture), which should however be correct if one replaces the Nisnevich topology by Lichtenbaum's topology.

What about this? As far as one is concerned by application to numbertheoretic conjectures like the Soulé conjecture or the Lichtenbaum conjectures, this is not very serious: concerning the orders of zeroes, the ranks will still be finite and almost always 0 , and the groups involved in special values are Lichtenbaum cohomology groups anyway. If one wants to get back a version of the Bass conjecture for algebraic $K$-theory, all one has to do is to define a "Lichtenbaum $K$-theory" similar to étale $K$-theory:

$$
K^{W}(X):=\mathbb{H}_{W}(X, \mathcal{K})
$$

where the notation means hypercohomology à la Thomason ([192]; see $\S 5$ in Geisser's chapter) for the Weil-étale topology.

On the other hand, it is quite amusing to remark that the étale topology, not the Zariski topology, shows up in the Lichtenbaum conjectures 6.6. In fact the Bloch-Kato or Beilinson-Lichtenbaum conjectures do not seem to play any rôle either in their formulation or in their (partial) proofs. (Even if we gave several examples where the Milnor conjecture gives vanishing or finiteness results, it was not used in the proofs of [95].) This also means that, in characteristic 0 , the correct formulation (for, say, the zeta function) most certainly involves an étale-related version of motivic cohomology. In small Krull dimension it may be replaced by plain motivic cohomology but this will not work from dimension 3 onwards, as one already sees in characteristic $p$. For rings of integers of number fields, the original Lichtenbaum formulation 6.4.1 led to the Quillen-Lichtenbaum and the Beilinson-Lichtenbaum conjectures and a huge development of algebraic $K$-theory and motivic cohomology. The Bloch-Kato conjecture is needed to prove it (in the cases one can) under this form. If it is indeed étale motivic cohomology rather than ordinary motivic cohomology that is relevant, all this work will have been the result of a big misunderstanding!

Let me give one nice consequence of Kato's conjecture 8.1.1, or rather of its partial proof by Colliot-Thélène-Suwa:

Theorem 8.1.2. Let $X$ be smooth projective of dimension $d$ over $\mathbf{F}_{p}$. Then the map

$$
C H^{d}(X) \rightarrow H_{\text {ét }}^{2 d}(X, \mathbf{Z}(d))
$$

is bijective. 
Proof. For $d=2$, this follows from the short exact sequence [86]

$$
0 \rightarrow C H^{2}(X) \rightarrow H_{\text {ét }}^{4}(X, \mathbf{Z}(2)) \rightarrow H^{0}\left(X, \mathcal{H}^{3}(\mathbf{Q} / \mathbf{Z}(2))\right) \rightarrow 0
$$

and Kato's theorem. In general, consider the coniveau spectral sequence for étale motivic cohomology

$$
E_{1}^{p, q}=\bigoplus_{x \in X^{(p)}} H_{\text {ét }}^{q-p}(k(x), \mathbf{Z}(d-p)) \Rightarrow H_{\text {ét }}^{p+q}(X, \mathbf{Z}(d)) .
$$

We have $E_{1}^{p, q}=0$ for $p>d$ for dimension reasons. For $q>d$ there are exact sequences

$H_{\text {ét }}^{q-p-1}(k(x), \mathbf{Q} / \mathbf{Z}(d-p)) \rightarrow H_{\text {ét }}^{q-p}(k(x), \mathbf{Z}(d-p)) \rightarrow H_{\text {êt }}^{q-p}(k(x), \mathbf{Q}(d-p))$.

By Theorem 4.2.1, the last group is 0 . The first is 0 pour $q-1>d+1$ for cohomological dimension reasons. Hence $E_{1}^{p, q}=0$ for $q \geq d+3$. Moreover, $E_{1}^{d-1, d+1}=E_{1}^{d-2, d+1}=0$ by Hilbert 90 and Hilbert 90 in weight 2 (MerkurjevSuslin theorem). Finally, $E_{2}^{d-2, d+2}=0$ by the Colliot-Thélène-Suwa theorem. Hence $E_{2}^{p, 2 d-p}=0$ except for $p=d$ and there are no differentials arriving to $E_{2}^{d, d}=C H^{d}(X)$. The proof is complete.

\subsection{Characteristic 0}

In characteristic 0 , things are considerably more complicated. If we start with the Beilinson conjecture 6.5.2, its analogue for smooth projective $\mathbf{Q}$-varieties predicts a two-layer filtration on their Chow groups; cycles homologically (i.e., conjecturally, numerically) equivalent to 0 should be detected by an Abel-Jacobi map to Deligne-Beilinson cohomology.

Concerning the Tate-Beilinson Conjecture 6.7.2, the only thing I can do is to conjecture that there is a conjecture.

Conjecture 8.2.1. There is a conjecture in the form of that in Theorem 6.8.3 in characteristic 0 , where the left hand side is a form of motivic cohomology and the right hand side is a form of an absolute cohomology theory in the sense of Beilinson [11].

Presumably the left hand side would be motivic hypercohomology with respect to the "Weil topology in characteristic 0" that Lichtenbaum is currently developing. As for the right hand side, I feel that it should probably be a mixture of the various (absolute counterparts of the) classical cohomology theories: $l$-adic, Betti, de Rham, $p$-adic, so as to involve the comparison isomorphisms. This formulation should be as powerful as in characteristic $p$ and account for most conjectures in characteristic 0 .

This being said, there is a basic problem to start the construction: if we take the $l$-adic cohomology of a ring of integers $\mathcal{O}_{S}$ (in which $l$ is invertible), 
it is nonzero even for negative Tate twists: by Tate and Schneider [164] we have

$$
\sum_{i=0}^{2}(-1)^{i+1} \operatorname{dim}_{\mathbf{Q}_{l}} H^{i}\left(\mathcal{O}_{S}, \mathbf{Q}_{l}(n)\right)= \begin{cases}r_{2} & \text { if } n \text { is even } \\ r_{1}+r_{2} & \text { if } n \text { is odd }\end{cases}
$$

hence there is no chance to compare it in the style of Theorem 4.2.7 with motivic cohomology, which vanishes in negative weights. The first thing to do would be to modify $l$-adic cohomology in order to correct this phenomenon: although this is clearly related to real and complex places, I have no idea how to do this. Note that Lichtenbaum's theory will be for Arakelov varieties.

One definitely needs new insights in order to follow this line of investigation!

\section{References}

1. S. S. Abyankhar Resolution of singularities of embedded algebraic surfaces (2nd ed.), Springer, 1998.

2. Y. André Pour une théorie inconditionnelle des motifs, Publ. Math. IHÉS $\mathbf{8 3}$ (1996), 5-49.

3. Y. André Une introduction aux motifs, book in preparation.

4. Y. André Motifs de dimension finie (d'après S.I. Kimura, P. O'Sullivan...), Sém. Bourbaki, Exposé no 929, March 2004.

5. Y. André, B. Kahn Construction inconditionnelle de groupes de Galois motiviques, C. R. Acad. Sci. Paris 334 (2002), 989-994.

6. Y. André, B. Kahn Nilpotence, radicaux et structures monoïdales (with an appendix of P. O'Sullivan), Rendiconti Sem. Math. Univ Padova 108 (2002), 107291.

7. R. Akhtar Zero-cycles on varieties over finite fields, Comm. Algebra 32 (2004), 279-294.

8. M. Artin, B. Mazur Étale homotopy, Lect. Notes in Math. 100, Springer, 1969.

9. J. Ayoub Formalisme des 4 opérations, preprint, 2004, http://www . math.jussieu.fr/ ayoub

10. A. A. Beilinson Higher regulators and values of L-functions (in Russian), Modern Problems in Mathematics VINITI Ser. 24 (1984), 181-238.

11. A. A. Beilinson Notes on absolute Hodge cohomology, in Applications of algebraic $K$-theory to algebraic geometry and number theory, Contemp. Math. $\mathbf{5 5}$ Part I, II, AMS, 1986, 35-68.

12. A. A. Beilinson Height pairings between algebraic cycles, Lect. Notes in Math. 1289, Springer, 1987, 1-26.

13. A. A. Beilinson On the derived category of perverse sheaves, Lect. Notes in Math. 1289, Springer, 1987, 27-41.

14. A. Beilinson, R. MacPherson, V. Schechtman Note on motivic cohomology, Duke Math. J. 54 (1987), 679-710.

15. J.-R. Belliard, T. Nguyen Quang Do, T. Formules de classes pour les corps abéliens réels, Ann. Inst. Fourier 51 (2001), 903-937.

16. D. Benois, T. Nguyen Quang Do Les nombres de Tamagawa locaux et la conjecture de Bloch et Kato pour les motifs $\mathbf{Q}(m)$ sur un corps abélien, Ann. Sci. Éc. Norm. Sup. 35 (2002), 641-672. 
17. B. J. Birch $K_{2}$ of global fields, in Proc. Sympos. pure Math. 20, AMS, 1970, 87-95.

18. S. Bloch Algebraic K-theory and classfield theory for arithmetic surfaces, Ann. of Math. 114 (1981), 229-265.

19. S. Bloch Lectures on algebraic cycles, Duke Univ. Lectures Series, 1982.

20. S. Bloch Algebraic cycles and higher K-theory, Adv. Math. 61 (1986), 267-304.

21. S. Bloch The moving lemma for higher Chow groups, J. Algebraic Geom. 3 (1994), 537-568.

22. S. Bloch, S. Lichtenbaum A spectral sequence for motivic cohomology, preprint, 1995, http://www .math.uiuc.edu/K-theory/62.

23. S. Bloch, I. Kriz Mixed Tate motives, Ann. of Math. 140 (1994), 557-605.

24. A. Borel Stable real cohomology of arithmetic groups, Ann. Sci. Ec. Norm. Sup. 7 (1974), 235-272.

25. A. Borel Cohomologie de $S L_{n}$ et valeurs de fonctions zêta aux points entiers, Ann. Scuola Norm. Sup. Pisa 4 (1977), 613-636; errata: ibid. , 7 (1980), 373.

26. A. Borel Values of zeta functions at integers, cohomology and polylogarithms, in Current trends in Mathematics and Physics (a tribute to Harish-Chandra), Narosa, 1995, 1-44.

27. A. Borel, J. Yang The rank conjecture for number fields, Math. Res. letters 1 (1994), 689-699.

28. L. Breen Tannakian categories, in Motives, Proc. Symp. pure Math. 55 (I) AMS, 1994, 337-376.

29. A. Bruguières On a tannakian result due to Nori, preprint of the Département de Mathématiques de l'Université Montpellier II.

30. G. J. I. Burgos The regulators of Borel and Beilinson, CRM Monograph Ser. 15, AMS, 2002.

31. J.-L. Colliot-Thélène On the reciprocity sequence in the higher class field theory of function fields, in Algebraic $K$-theory and algebraic topology, NATO ASI Ser. C Math. 407, Kluwer, 1993, 35-55.

32. J.-L. Colliot-Thélène, R. Hoobler, B. Kahn The Bloch-Ogus-Gabber theorem, Algebraic $K$-theory (Toronto, ON, 1996), Fields Inst. Commun., 16, AMS, 1997, 31-94.

33. K. Coombes Motifs, L-functions and the K-cohomology of rational surfaces over finite fields, Math. Ann. 276 (1987), 255-267.

34. P. Deligne La conjecture de Weil, II, Publ. Math. IHES 52 (1980), 137-252.

35. P. Deligne Hodge cycles on abelian varieties (notes by J. S. Milne), in Hodge cycles, motives and Shimura varieties, Lect. Notes in Math. 900, Springer, 1982, 9-100.

36. P. Deligne Le groupe fondamental de la droite projective moins trois points, in Galois groups over Q (Y. Ihara, K. Ribet, J.-P. Serre eds.), MSRI Publications 16, Springer, 1989, 79-297.

37. P. Deligne Catégories tannakiennes, in The Grothendieck Festschrift II, Progress in Math. 87, Birkhäuser, 1990, 111-195.

38. P. Deligne Décompositions dans la catégorie dérivée, in Motives, Proc. Symposia Pure Math. 55 (1), AMS, 1994, 115-128.

39. P. Deligne, J.S. Milne Tannakian categories, in Hodge cycles, motives and Shimura varieties, Lect. Notes in Math. 900, Springer, 1982, 101-228.

40. P. Deligne, K. Ribet Values of L-functions at negative integers over totally real fields, Invent. Math. 59 (1980), 227-286. 
41. M. Demazure Motifs de variétés algébriques, Sém. Bourbaki 365 1969/70, 19-38 (1971).

42. B. Dwork On the rationality of the zeta function of an algebraic variety, Amer. J. Math. 82 (1960), 631-648.

43. W. Dwyer, E. Friedlander, V. Snaith; R. Thomason Algebraic K-theory eventually surjects onto topological K-theory, Invent. Math. 66 (1982), 481-491.

44. W. Dwyer, E. Friedlander Algebraic and étale K-theory, Trans. AMS 292 (1985), 247-280.

45. W. Dwyer, E. Friedlander Some remarks on the K-theory of fields, in Applications of algebraic $K$-theory to algebraic geometry and number theory, Part I, II (Boulder, 1983), Contemp. Math., 55, AMS, 1986, 149-158.

46. G. Faltings, G. Wüstholz Rational points, Aspects of Math., Vieweg, 1986.

47. V. Fleckinger, M. Kolster, T. Nguyen Quang Do Twisted S-units, p-adic class number formulas and the Lichtenbaum conjecture, Duke Math. J. 84 (1996), 679-717; correction: ibid. , 90 (1997), 641-643.

48. E. Friedlander Étale K-theory. I. Connections with etale cohomology and algebraic vector bundles, Invent. Math. 60 (1980), 105-134.

49. E. Friedlander Étale K-theory, II. Connections with algebraic K-theory. Ann. Sci. Éc. Norm. Sup. 15 (1982), 231-256.

50. E. Friedlander Motivic complexes of Suslin and Voevodsky, Sém. Bourbaki 1996/1997, Exp. 833, Astérisque 245 (1997), 355-378. Reproduced in this Handbook.

51. E. Friedlander, A. Suslin The spectral sequence relating algebraic K-theory and motivic cohomology, Ann. Sci. Ec. Norm. Sup. 35 (2002), 773-875.

52. E. Friedlander, V. Voevodsky Bivariant cycle cohomology, in Cycles, transfers and motivic cohomology theories, Annals of Math. Studies 143, 2000, 138-187.

53. K. Fujiwara A proof of the absolute purity conjecture (after Gabber), in Algebraic geometry 2000, Azumino (Hotaka), Adv. Stud. Pure Math. 36, Math. Soc. Japan, Tokyo, 2002, 153-183.

54. W. Fulton Intersection theory, Springer, 1984.

55. O. Gabber Affine analog of the proper base change theorem, Israel J. Math. 87 (1994), 325-335.

56. T. Geisser Tate's conjecture, algebraic cycles and rational K-theory in characteristic $p, K$-Theory 13 (1998), 109-122.

57. T. Geisser Applications of de Jong's theorem on alterations, in Resolution of Singularities In tribute to Oscar Zariski, Progr. in Math. 181, Birkhäuser, 2000, 299-314.

58. T. Geisser Weil-étale motivic cohomology over finite fields, preprint, 2002, http: //www . math. uiuc. edu/K-theory/565.

59. T. Geisser Motivic cohomology over Dedekind rings, to appear in Math. Z.

60. T. Geisser Arithmetic cohomology over finite fields and special values of $\zeta$ functions, preprint, 2004.

61. T. Geisser, M. Levine The K-theory of fields in characteristic $p$, Invent. Math. 139 (2000), 459-493.

62. T. Geisser, M. Levine The Bloch-Kato conjecture and a theorem of SuslinVoevodsky, J. reine angew. Math. 530 (2001), 55-103.

63. H. Gillet Gersten's conjecture for the K-theory with torsion coefficients of a discrete valuation ring, J. Alg. 103 (1986), 377-380.

64. H. Gillet, M. Levine The relative form of Gersten's conjecture over a discrete valuation ring: the smooth case. J. Pure Appl. Algebra 46 (1987), 59-71. 
65. H. Gillet, C. Soulé Filtrations on higher algebraic K-theory, in Algebraic Ktheory, Proc. Symp. pure Math. 67, AMS, 1999, 89-148.

66. D. Grayson (after D. Quillen) Finite generation of $K$-groups of a curve over a finite field, Lect. Notes in Math. 966, Springer, 1982, 69-90.

67. D. Grayson Weight filtrations via commuting isomorphisms, K-theory 9 (1995), 139-172.

68. A. Grothendieck Standard conjectures on algebraic cycles, Algebraic Geometry - Bombay Colloquium, 1968, Oxford, 1969, 193-199.

69. A. Grothendieck, J.-P. Serre Correspondance, Documents mathématiques, SMF, 2001.

70. C. Haesemeyer Descent properties of homotopy K-theory, preprint, 2003 , http: //www.math. uiuc. edu/K-theory/650.

71. M. Hanamura Mixed motives and algebraic cycles, I, II, III, I: Math. Res. Lett. 2 (1995), 811-821; II: preprint, 1996; III: Math. Res. Lett. 6 (1999), 61-82.

72. M. Hanamura Homological and cohomological motives of algebraic varieties, Invent. Math. 142 (2000), 319-349.

73. G. Harder Die Kohomologie S-arithmetischer Gruppen über Funktionenkörpern, Invent. Math. 42 (1977), 135-175.

74. R. Hoobler The Merkurjev-Suslin theorem for arbitrary semi-local rings, preprint, 1993.

75. M. Hopkins, F. Morel Article in preparation.

76. U. Jannsen Continuous étale cohomology, Math. Ann. 280 (1988), 207-245.

77. U. Jannsen On the l-adic cohomology of varieties over number fields and its Galois cohomology, MSRI Publications 16, Springer, 1989, 315-360.

78. U. Jannsen Mixed motives and algebraic $K$-theory, Lect. Notes in Math. 1400, Springer, 1990.

79. U. Jannsen Motives, numerical equivalence and semi-simplicity, Invent. Math. 107 (1992), 447-452.

80. U. Jannsen Letter to Katz and Messing, July 30, 1991.

81. U. Jannsen Motivic sheaves and filtrations on Chow groups, in Motives, Proc. Symposia Pure Math. 55 (1), AMS, 1994, 245-302.

82. A. J. de Jong Smoothness, semi-stability and alterations, Publ. Math. IHÉS 83 (1996), 51-93.

83. B. Kahn Some conjectures in the algebraic K-theory of fields, I: K-theory with coefficients and étale K-theory, NATO ASI Series, Ser. C. 279, Kluwer, 1989, 117-176.

84. B. Kahn $K_{3}$ d'un schéma régulier, C. R. Acad. Sci. Paris 315 (1992), 433-436.

85. B. Kahn On the Lichtenbaum-Quillen conjecture, Algebraic $K$-theory and algebraic topology (Lake Louise, AB, 1991), NATO ASI Ser. C 407, Kluwer, 1993, $147-166$.

86. B. Kahn Applications of weight-two motivic cohomlogy, Doc. Math. J. DMV 1 (1996), 395-416.

87. B. Kahn The Bass conjecture implies the Beilinson-Soulé conjecture, and more, preprint, 1996.

88. B. Kahn The Quillen-Lichtenbaum conjecture at the prime 2, preprint, 1997, http://www . math. uiuc.edu/K-theory/208.

89. B. Kahn La conjecture de Milnor (d'après V. Voevodsky, Sém. Bourbaki 1996/1997, Exp. 834, Astérisque 245 (1997), 379-418. Reproduced in this Handbook. 
90. B. Kahn A sheaf-theoretic reformulation of the Tate conjecture, preprint of the Institute de Mathématiques de Jussieu no 150, 1998, math.AG/9801017.

91. B. Kahn K-theory of semi-local rings with finite coefficients and étale cohomology, K-theory 25 (2002), 99-139.

92. B. Kahn The Geisser-Levine method revisited, preliminary version of [93], http://www. math.uiuc. edu/K-theory/438.

93. B. Kahn The Geisser-Levine method revisited and algebraic cycles over a finite field, Math. Ann. 324 (2002), 581-617.

94. B. Kahn Some finiteness results in étale cohomology, J. Number Theory 99 (2003), 57-73.

95. B. Kahn Équivalences rationnelle et numérique sur certaines variétés de type abélien sur un corps fini, Ann. Sci. Ec. Norm. Sup. 36 (2003), 977-1002.

96. B. Kahn On the semi-simplicity of Galois actions, to appear in the Rendiconti Sem. Mat. Univ. Padova 112 (2004).

97. B. Kahn Sur le groupe des classes d'un schéma arithmétique (with an appendix by M. Hindry), preprint, 2003.

98. W. van der Kallen Homology stability for linear groups, Invent. Math. 60 (1980), 269-295.

99. M. Karoubi $K$-theory, an introduction, Springer, 1978.

100. K. Kato A generalization of higher class field theory by using K-groups, I, J. Fac. Sci., Univ. Tokyo 26 (1979), 303-376.

101. K. Kato A Hasse principle for two-dimensional global fields, with an appendix by J.-L. Colliot-Thélène, J. Reine Angew. Math. 366 (1986), 142-183.

102. K. Kato, S. Saito Unramified class field theory of arithmetical surfaces, Ann. of Math. 118 (1983), 241-275.

103. T. Katsura, T. Shioda On Fermat varieties, Tôhoku Math. J. 31 (1979), 97115.

104. N. Katz, W. Messing Some consequences of the Riemann hypothesis for varieties over finite fields, Invent. Math. 23 (1974), 73-77.

105. N. Katz, W. Messing Some remarks on the Tate conjecture over finite fields and its relations to other conjectures, manuscript notes, July 30, 1991.

106. S.I. Kimura Chow motives can be finite-dimensional, in some sense, preprint, rev. 2004.

107. S. Kleiman Algebraic cycles and the Weil conjectures, in Dix exposés sur la cohomologie des schémas, North-Holland, Amsterdam, 1968, 359-386.

108. S. Kleiman Motives, in Algebraic geometry - Oslo, 1970, Sijthoff \& Noordhoff 1970, 53-62.

109. S. Kleiman The standard conjectures, in Motives, Proc. Symp. pure Math. 55 (I), AMS, 1994, 3-20.

110. M. Kolster Higher relative class number formulae, Math. Ann. 323 (2002), 667-692.

111. M. Kolster K-theory and arithmetic, in Contemporary developments in algebraic $K$-theory (M. Karoubi, A.O. Kuku, C. Pedrini, eds.), ICTP Lecture Notes 15 (2003), the Abdus Salam International Centre for Theoretical Physics, 191258.

112. H. W. Lenstra, Y.G. Zarhin The Tate conjecture for almost ordinary abelian varieties over finite fields, Advances in number theory (Kingston, ON, 1991), Oxford Univ. Press, 1993, 179-194.

113. M. Levine The indecomposable $K_{3}$ of fields, Ann. Sci. Éc. Norm. Sup. 22 (1989), $255-344$. 
114. M. Levine Relative Milnor K-theory, K-Theory 6 (1992), 113-175.

115. M. Levine Tate motives and the vanishing conjectures for algebraic $K$-theory, in Algebraic $K$-theory and algebraic topology (Lake Louise, 1991), NATO ASI Series, Ser. C 407, Kluwer, 1993, 167-188.

116. M. Levine Bloch's higher Chow groups revisited, in K-theory (Strasbourg, 1992), Astérisque 226 (1994), 235-320.

117. M. Levine Lambda operations, K-theory and motivic cohomology, in Algebraic $K$-theory (Toronto, 1996), Fields Inst. Comm. Series 16 AMS, 1997, 131-184.

118. M. Levine Mixed motives, Math. Surveys and Monographs 57, AMS, Providence, 1998.

119. M. Levine Techniques of localization in the theory of algebraic cycles, J. Alg. Geom. 10 (2001), 299-363.

120. M. Levine K-theory and motivic cohomology of schemes, preprint, 1999, http://www . math. uiuc. edu/K-theory/336.

121. M. Levine K-theory and motivic cohomology of schemes, I, preprint, 2002.

122. M. Levine The homotopy coniveau filtration, preprint, 2003, http://www.math.uiuc.edu/K-theory/628.

123. S. Lichtenbaum Values of zeta functions, étale cohomology and algebraic $K$ theory, Lect. Notes in Math. 341, Springer, 1972, 489-501.

124. S. Lichtenbaum Values of zeta functions at non-negative integers, Lect. Notes in Math. 1068, Springer, 1984, 127-138.

125. S. Lichtenbaum The construction of weight-two arithmetic cohomology, Invent. Math. 88 (1987), 183-215.

126. S. Lichtenbaum The Weil-étale topology, preprint, 2001.

127. D. Lieberman Numerical and homological equivalence of algebraic cycles on Hodge manifolds, Amer. J. Math 90 (1968), 366-374.

128. Yu. Manin Correspondences, motifs and monoidal transformations (in Russian), Engl. Transl.: Math. USSR Sbornik 6 (1968), 439-470.

129. H. Matsumoto Sur les sous-groupes arithmétiques des groupes semi-simples déployés, Ann. Sci. Éc. Norm. Sup. 2 (1969), 1-62.

130. B. Mazur, A. Wiles Class fields of abelian extensions of $\mathbf{Q}$, Invent. Math. 76 (1984), 179-330.

131. A. S. Merkurjev, A. A. Suslin K-cohomology of Severi-Brauer varieties and the norm residue homomorphism (in Russian). English transl.: Math. USSR Izv. 21 (1983), 307-340.

132. A. S. Merkurjev, A. A. Suslin The norm residue symbol of degree 3 (in Russian). English transl.: Math. USSR Izv. 36 (1991), 349-367.

133. A. S. Merkurjev, A. A. Suslin The group $K_{3}$ for a field (in Russian). English transl.: Math. USSR Izv. 36 (1991), 541-565.

134. J. S. Milne Étale cohomology, Princeton University Press, 1980.

135. J. S. Milne Abelian varieties, in Arithmetic Geometry (G. Cornell, J. Silverman, eds.), Springer, 1986, 103-150.

136. J. S. Milne Arithmetic duality theorems, Perspectives in Math. 1, Acad. Press, 1986.

137. J. S. Milne Motivic cohomology and values of the zeta function, Compositio Math. 68 (1988), 59-102.

138. J. S. Milne Motives over finite fields, in Motives (Seattle, WA, 1991), Proc. Sympos. Pure Math., 55, Part 1 (1994), Amer. Math. Soc., Providence, RI, 401-459. 
139. J. S. Milne The Tate conjecture for certain abelian varieties over finite fields, Acta Arith. 100 (2001), 135-166.

140. J. Milnor Algebraic K-theory and quadratic forms, Invent. Math. 9 (1970), 315-344.

141. A. Mokrane Cohomologie cristalline des variétés ouvertes, Maghreb Math. Rev. 2 (1993), 161-175.

142. F. Morel Voevodsky's proof of Milnor's conjecture, Bull. Amer. Math. Soc. 35 (1998), 123-143.

143. F. Morel Théorie homotopique des schémas, Astérisque 256 (1999).

144. F. Morel An introduction to $\mathbf{A}^{1}$-homotopy theory, in Contemporary developments in algebraic $K$-theory (M. Karoubi, A.O. Kuku, C. Pedrini, eds.), ICTP Lecture Notes 15 (2003), the Abdus Salam International Centre for Theoretical Physics, 357-441.

145. F. Morel On the motivic $\pi_{0}$ of the sphere spectrum, in Axiomatic, Enriched and Motivic Homotopy Theory (J.P.C Greenlees, ed.), NATO Science Series II 131 (2004), Kluwer, 219-260.

146. F. Morel, V. Voevodsky $\mathbf{A}^{1}$-homotopy of schemes, Publ. Math. IHÉS 90 (1999), 45-143 (2001).

147. D. Mumford Abelian varieties, TIFR Studies in Math. 5, Oxford Univ. Press, 1970.

148. Yu. Nesterenko, A. Suslin Homology of the general linear group and Milnor K-theory (in Russian), Izv. Akad. Nauk SSSR 53 (1989), 121-146.

149. M. Nori Constructible sheaves, in Algebra, Arithmetic and Geometry (II), TIFR, Mumbai, Narosa/AMS, 2002, 471-491.

150. P. O'Sullivan Letters to Y. André and B. Kahn, April 29 and May 12, 2002.

151. P. A. Østvær, A. Rosenschon The homotopy limit problem for two-primary algebraic K-theory, preprint, 2002.

152. D. Quillen On the cohomology and $K$-theory of the general linear group over a finite field, Ann. of Math. 96 (1972), 552-586.

153. D. Quillen Higher algebraic K-theory, I, Lecture Notes in Mathematics 341, Springer, Berlin, 1973, 77-139.

154. D. Quillen Finite generation of the groups $K_{i}$ of rings of algebraic integers, Lect. Notes in Math. 341, Springer, New York, 1972, 179-198.

155. D. Quillen Higher algebraic K-theory, Proceedings of the International Congress of Mathematicians (Vancouver, 1974), Vol. 1, 171-176. Canad. Math. Congress, Montreal, 1975.

156. M. S. Raghunathan A note on quotients of real algebraic groups by arithmetic subgroups, Invent. Math. 4 (1968), 318-335.

157. M. Rapoport Comparison of the regulators of Beilinson and of Borel, in Beilinson's conjectures on special values of $L$-functions (M. Rapopoart, N. Schappacher, P. Schneider, eds.), Perspectives in Math. 4, Acad. Press, 1988.

158. J. Riou Théorie homotopique des $S$-schémas, mémoire de DEA (in French), Paris 7, 2002, http://www.math.jussieu.fr/ riou/dea/.

159. J. Rognes, C. Weibel Two-primary algebraic K-theory of rings of integers in number fields, J. Amer. Math. Soc. 13 (2000), 1-54.

160. M. Rost Hilbert's theorem 90 for $K_{3}^{M}$ for degree-two extensions, preprint, 1996.

161. M. Rost Norm varieties and algebraic cobordism, Proc. ICM 2002, Higher Education Press, Beijing, Vol. II, 77-85.

162. N. Saavedra Rivano Catégories tannakiennes, Lect. Notes in Math. 265, Springer, 1972 . 
163. T. Saito Weight spectral sequences and independence of $l$, J. Inst. Math. Jussieu 2 (2003), 583-634.

164. P. Schneider Über gewisse Galoiscohomologiegruppen, Math. Z. 168 (1979), 181-205.

165. C. Schoen Complex varieties for which the Chow group mod $n$ is not finite, J. Alg. Geom. 11 (2002), 41-100.

166. A. Scholl Classical motives, in Motives, Proc. Symp. pure Math. 55 (I), AMS, 1994, 163-187.

167. J.-P. Serre Résumé des cours de 1960-61, Ann. Collège de France, 51-52 (1961) $=$ CEuvres, Vol. II, 150-151.

168. J.-P. Serre Zeta and L functions, in Arithmetical Algebraic Geometry, Harper and Row (1965), 82-92 = CEuvres, Vol. II, 249-259.

169. J.-P. Serre Facteurs locaux des fonctions zêta des variétés algébriques: définitions et conjectures, Sém. Delange-Pisot-Poitou, 1969/70, Exp. no $19=$ (Euvres, Vol. II, 581-592.

170. J. P. Serre Cohomologie des groupes discrets, in Prospects in Mathematics, Ann. Math. Studies 70, Princeton Univ. Press, 1971, 77-169 = Cuvres, Vol II, 593-685.

171. V. Snaith Algebraic cobordism and $K$-theory, Mem. Amer. Math. Soc. 21 (1979), no. 221.

172. C. Soulé $K$-théorie des anneaux d'entiers de corps de nombres et cohomologie étale, Invent. Math. 55 (1979), 251-295.

173. C. Soulé On higher $p$-adic regulators, Lect. Notes in Math. 854, Springer, 1981, 372-399.

174. C. Soulé K-théorie et zéros aux points entiers de fonctions zêta, Proc. ICM 1983, I, Polish Scientific Publishers, Warszawa, 1984, 437-445.

175. C. Soulé Groupes de Chow et $K$-théorie de variétés sur un corps fini, Math. Ann. 268 (1984), 317-345.

176. C. Soulé Opérations en K-théorie algébrique, Can. Math. J. 37 (1985), 488550 .

177. C. Soulé Letter to Bloch and Lichtenbaum, 8-2-1995.

178. M. Spieß Proof of the Tate conjecture for products of elliptic curves over finite fields, Math. Ann. 314 (1999), 285-290.

179. M. Spitzweck Operads, algebras and modules in model categories and motives, PhD thesis, Bonn, 2001.

180. A. M. Šermenev Motif of an Abelian variety (in Russian); English translation: Functional Anal. Appl. 8 (1974), 47-53.

181. A. Suslin Algebraic K-theory of fields, Proc. ICM 1996, Berkeley, 1986, 222244 .

182. A. Suslin The Beilinson spectral sequence for the K-theory of the field of real numbers (in Russian), Mat. Metody i Fiz.-Mekh. Polya 28 (1988), 51-52, 105; English transl.: J. Soviet Math. 63 (1993), 57-58.

183. A. Suslin Voevodsky's proof of the Milnor conjecture, in Current developments in mathematics, 1997, Int. Press, 1999, 173-188.

184. A. Suslin On the Grayson spectral sequence, preprint, 2003, http://www . math. uiuc.edu/K-theory/588.

185. A. Suslin, V. Voevodsky Singular homology of abstract algebraic varieties, Invent. Math. 123 (1996), 61-94. 
186. A. Suslin, V. Voevodsky Bloch-Kato conjecture and motivic cohomology with finite coefficients, in The arithmetic and geometry of algebraic cycles (Banff, AB, 1998), 117-189, NATO Sci. Ser. C Math. Phys. Sci., 548, Kluwer Acad. Publ., Dordrecht, 2000.

187. N. Suwa A note on Gersten's conjecture for logarithmic Hodge-Witt sheaves, $K$-Theory 9 (1995), no. 3, 245-271.

188. J. T. Tate Algebraic cycles and poles of zeta functions, Arithmetical Algebraic Geometry (Proc. Conf. Purdue Univ., 1963), 1965, 93-110.

189. J.T. Tate Endomorphisms of abelian varieties over finite fields, Invent. Math. 2 (1966), 134-144.

190. J. T. Tate Symbols in arithmetic, Proc. ICM, Nice, 1970, 201-211.

191. J. T. Tate Conjectures on algebraic cycles in l-adic cohomology, in Motives, Proc. Symposia Pure Math. 55 (1), AMS, 1994, 71-83.

192. R. Thomason Algebraic K-theory and étale cohomology, Ann. Sci. Ec. Norm. Sup. 13 (1985), 437-452.

193. R. Thomason Absolute cohomological purity, Bull. Soc. Math. France 112 (1984), 397-406.

194. R. Thomason Survey of algebraic vs. étale topological K-theory, in Algebraic $K$-theory and algebraic number theory (Honolulu, 1987), Contemp. Math. 83, AMS, 1989, 393-443.

195. R. Thomason, T. Trobaugh Higher algebraic K-theory of schemes and of derived categories, in The Grothendieck Festschrift II, Progress in Math. 87, Birkhäuser, 1990, 247-435.

196. B. Totaro Milnor's K-theory is the simplest part of algebraic K-theory, Ktheory 6 (1992), 177-189.

197. V. Voevodsky, A nilpotence theorem for cycles algebraically equivalent to zero, Int. Mat. Res. Notices 4 (1995), 1-12.

198. V. Voevodsky Triangulated categories of motives over a field, in Cycles, transfers and motivic cohomology theories, Annals of Math. Studies 143, 2000, 188 238.

199. V. Voevodsky Lectures on K-theory and motivic cohomology (notes by C. Weibel), Proc. Sympos. Pure Math., 67, Algebraic K-theory (Seattle, 1997), 283-303, AMS, 1999.

200. V. Voevodsky Motivic cohomology groups are isomorphic to higher Chow groups in any characteristic, Int. Math. Res. Notices 2002, 331-355.

201. V. Voevodsky $\mathbf{A}^{1}$-homotopy theory, Proc. ICM 1998, Doc. Math. Extra Volume I, 1998, 579-604.

202. V. Voevodsky Cancellation theorem, preprint, 2002, http: //www . math. uiuc. edu/K-theory/541.

203. V. Voevodsky Motivic cohomology with Z/2 coefficients, to appear in Publ. Math. IHÉS.

204. V. Voevodsky Lectures on cross functors, Fall 2001 (notes by P. Deligne), Motivic homotopy theory program: http://www.math.ias. edu/ vladimir/seminar.html

205. V. Voevodsky On motivic cohomology with $\mathbf{Z} / l$ coefficients, preliminary version, June 16, 2003, http://www . math.uiuc.edu/K-theory/639.

206. R. Vogt Homotopy limits and colimits, Math. Z. 134 (1973), 11-52.

207. F. Waldhausen Algebraic K-theory of spaces, Algebraic and Geometric Topology, Lect. Notes in Math. 1126, Springer, 1985, 318-419. 
208. C. Weibel Mayer-Vietoris sequences and mod $p$ K-theory, in Algebraic $K$ theory: Oberwolfach 1980, Lect. Notes in Math. 966 (1982), Springer, 390-407.

209. C. Weibel Homotopy algebraic K-theory, Contemp. Math. 83 (1989), 461-488.

210. C. Weibel A road map of motivic homotopy and homology theory, preprint, 2003, http://www.math.uiuc.edu/K-theory/630.

211. A. Wiles The Iwasawa conjecture for totally real fields, Annals of Math. 131 (1990), 493-540.

212. Y.G. Zarhin Abelian varieties of K3 type, Séminaire de théorie des nombres, Paris, 1990-91, Progr. Math. 108, Birkhäuser, 1992, 263-279.

[EGA IV] A. Grothendieck, J. Dieudonné, Éléments de géométrie algébrique, chapitre IV: Étude locale des schémas et des morphismes de schémas (2ème partie), Publ. Math. IHES 24, 1965.

[SGA 4] A. Grothendieck, M. Artin, J.-L. Verdier Théorie des topos et cohomologie étale des schémas (3ème partie), Lect. Notes in Math. 305, Springer, 1973.

[SGA 4 1/2] P. Deligne et al. Cohomologie étale (SGA 4 1/2), Lect. Notes in Math. 569, Springer, 1977.

[SGA 5] A. Grothendieck et al. Cohomologie $l$-adique et fonctions $L$ (SGA 5), Lect. Notes in Math. 589, Springer, 1977. 


\title{
Index
}

\author{
Chern character \\ in algebraic geometry 10 \\ in topology 4 \\ Cohomology \\ continuous étale 47 \\ $l$-adic 39 \\ Lichtenbaum (Weil-étale) 52 \\ motivic $10,11,21$ \\ Conjecture \\ Bass 35 \\ Beilinson 44 \\ Beilinson-Lichtenbaum 26 \\ Beilinson-Parshin 44 \\ Beilinson-Soulé 13 \\ Bloch-Beilinson-Murre 20 \\ Bloch-Kato 26 \\ Kato 62
}

\author{
Kimura-O'Sullivan 60 \\ Lichtenbaum 41, 45 \\ Lichtenbaum-Quillen 7 \\ Merkurjev-Suslin 15 \\ Quillen 6 \\ Soulé 40 \\ Standard 17 \\ Tate 44 \\ Tate-Beilinson 48 \\ Voevodsky 18 \\ Milnor $K$-groups 6 \\ Spectral sequence \\ Atiyah-Hirzebruch 4 \\ Bloch-Lichtenbaum et al. 10
}

Zeta function 39 
